Federal Reserve Bank of Dallas

Globalization and Monetary Policy Institute

Working Paper No. 105

http://www.dallasfed.org/assets/documents/institute/wpapers/2012/0105.pdf

\title{
Bayesian Estimation of NOEM Models: Identification and Inference in Small Samples ${ }^{*}$
}

\author{
Enrique Martínez-García \\ Federal Reserve Bank of Dallas \\ Diego Vilán \\ University of Southern California \\ Mark Wynne \\ Federal Reserve Bank of Dallas \\ First Version: January 2012 \\ Revised: March 2012
}

\begin{abstract}
The global slack hypothesis (e.g., Martínez-García and Wynne (2010)) is central to the discussion of the trade-offs monetary policy faces in an increasingly more open world economy. Open-Economy (forward-looking) New Keynesian Phillips curves describe how expected future inflation and a measure of global output gap (global slack) affect the current inflation rate. This paper studies the (potential) weak identification of these relationships in the context of a fully-specified structural model using Bayesian estimation techniques. We trace the problems to sample size, rather than misspecification bias. We conclude that standard macroeconomic time series with a coverage of less than forty years are subject to potentially serious identification issues, and also to model selection errors. We recommend estimation with simulated data prior to bringing the model to the actual data as a way of detecting parameters that are susceptible to weak identification in short samples.
\end{abstract}

JEL codes: C11, C13, F41

\footnotetext{
* Enrique Martínez-García, Federal Reserve Bank of Dallas, Research Department, 2200 N. Pearl Street, Dallas, TX 75201. 214-922-5262. Enrique.martinez-garcia@dal.frb.org. Diego Vilán, University of Southern California, KAP 300, University Park Campus, Los Angeles, CA 90089. 213-740-8335. Diego.vilan@gmail.com. Mark A. Wynne, Federal Reserve Bank of Dallas, Research Department, 2200 N. Pearl Street, Dallas, TX 75201. 214-922-5159. Mark.a.wynne@dal.frb.org. We would like to thank Nathan Balke, María Teresa Martínez-García and participants in the $10^{\text {th }}$ annual Advances in Econometrics Conference for helpful suggestions. All remaining errors are ours alone. The views in this paper are those of the authors and do not necessarily reflect the views of the Federal Reserve Bank of Dallas or the Federal Reserve System.
} 


\section{Introduction}

Economic theory tells us that globalization-greater openness to trade, capital and labor flows - should matter for more than how much countries trade with each other. Martínez-García (2008) and MartínezGarcía and Wynne (2010), building on recent developments in the New Open Economy Macro (NOEM) literature (see, e.g., Clarida et al. (2002)) develop a tractable structural framework to evaluate the nature of the relationship between global slack and the cyclical component of inflation (the so-called global slack hypothesis). The main postulate of the model is that globalization may have altered the cyclical behavior of inflation simply by changing the composition of the basket of goods - from which the aggregate price indexes are calculated - and its origin. ${ }^{1}$

The work of Martínez-García and Wynne (2010) also raises questions about the potential estimation biases in detecting the effects of globalization with partial equilibrium, reduced-form regressions. Partial equilibrium analysis misses potentially important cross-equation restrictions that could result in misleading empirical inferences. To that, however, we must add the problem that the economically relevant concepts may not be observable - but model-dependent - and that the use of estimation techniques may be limited by data availability on related observables. In the model investigated by Martínez-García and Wynne (2010), as in most New Keynesian-type models, the key variables that we do not observe are the Home and Foreign output gaps. The emphasis of that paper is, therefore, placed on the poor quality of the statistical proxies for local or global slack often used in the empirical literature. ${ }^{2}$

Ultimately, the evaluation of Martínez-García and Wynne (2010) suggests that the validity of the global slack hypothesis - or, more broadly, the role of globalization in the cyclical component of U.S. inflationcannot be determined solely on the basis of simple least squares regressions on reduced-form (partial equilibrium) relationships of the sort reported in certain strands of the empirical literature. A fuller evaluation of the importance of openness for inflation dynamics seemingly requires a more structural approach to the multiple factors and diverse channels influencing the economy that can be taken to the data and tested. ${ }^{3}$

In this paper we focus on estimation implementation, as identification is a serious concern even when researchers are confident about their model's accurate characterization of the DGP process. To do that, we calibrate the model of Martínez-García and Wynne (2010) and use it as our DGP process. ${ }^{4}$ We explore the challenges of estimation on data simulated from that model. A large number of the existing papers on estimating open eocnomy models - and Adolfson et al. (2008) is no exception — rely on Bayesian techniques. Classical methods can also be used, but classical inference is conditional on the estimation of the parameters. In contrast, the treatment of unobserved variables (output gaps) and parameters as jointly distributed random variables means that with the Bayesian estimationapproach, estimates of each appropriately refflect uncertainty about the others - that is one of the powerful reasons behind the appeal and popularity of Bayesian estimation. More so in models like the one we investigate here where some of the key variables (the output gaps) are, indeed, best thought as unobserved.

\footnotetext{
${ }^{1}$ Other channels through which globalization may operate have been explored in the literature as well. For example, Engler (2009) investigates the implications of immigration on the Phillips curve relationship, while Rumler (2007) and Leith and Malley (2007) examine the effects of imported intermediate goods.

${ }^{2}$ A similar point is made in the closed-economy context by Neiss and Nelson (2003) and Neiss and Nelson (2005).

${ }^{3}$ Adolfson et al. (2005), Adolfson et al. (2007) and Adolfson et al. (2008) are among the recent papers that have attempted a more structural approach to open-economy economy issues based on estimation.

${ }^{4}$ To be more precise, we work with a slightly simplified version of the model which assumes countries have equal size (i.e., $n=\frac{1}{2}$ ) and home-product bias in consumption is symmetric in both countries (i.e., $\xi^{*}=(1-\xi)$ ).
} 
Bayesian methods have a long history in econometrics - the seminal work is Zellner (1971). Classical inference is based only on sample information and thus is purely objective, whereas one can take advantage of subjective and/or nonsample prior information for Bayesian inference. Bayesian methods combine the prior distribution with the likelihood function to form a posterior density. The choice of the prior distribution might be problematic along several dimensions and, most importantly, the estimation can be sensitive to the prior selected. Hence, how to elicit priors effectively (or how to constraint the subjective component in prior-selection) is a significant concern in applied work that we address in this paper.

If accounting for parameter uncertainty in the estimation of the unobserved variables (such as the output gap) is one of the advantages of Bayesian estimation, the choice of observable variables used in the estimation might be important to help us identify more or less precisely the estimated parameters or to infer the unobservable output gap variables. This latter point is of particular importance in estimating open-economy models because lack of data or the poor quality of the data available for most countries around the world restricts the time series of macro data that can be used in the estimation.

Since the majority of the literature on Bayesian estimation of Dynamic Stochastic General Equilibrium (DSGE) models has focused on a closed economy set-up or small-open economy models, we seek to make a contribution by assessing the "art" of eliciting priors and choosing observable variables to address the fundamental questions about the role of openness posed by the workhorse NOEM model. Our aim is to provide a practitioners' viewpoint on the complexities of estimating open-economy models, which is a subjec that has been relatively less documented than for closed-economy models. We will employ a variant of the two-country model of Martínez-García and Wynne (2010) to derive a benchmark specification - a DGPfor the open economy. Working with simulated data from a fully-specified model rather than actual data allows us a more precise assessment of the performance of the Bayesian technique and its sensitivity to implementation, as we would know at every step whether the model is well-specified and which properties it must satisfy. We use this version of the workhorse NOEM model to illustrate the challenges of estimating an open-economy model with regards to two fundamental questions: (1) which priors should used?; and (2) which observable variables should be considered?

In terms of the first question, eliciting priors to estimate the parameters of the model can have an effect on the estimate of the unobservables. There are a number of recurrent problems that a researcher must be aware of. First, we analyze and discuss parameter identification. Even when estimating a model that is linear in its variables, the structural parameters may enter in a highly non-linear form. In some cases, that would make it impossible to estimate all the structural parameters of the model independently. That forces the researcher to either estimate reduced-form parameters - giving up on the possibility of attaching a structural interpretation to those estimates - or to design an identification strategy that often involves the calibration of some of those structural parameters - but can be model-dependent. ${ }^{5}$ In general, it pays to know one's model before estimating it. However, we also document how the estimation can be biased whenever those parameterized values are off-the-mark, and discuss the implications of doing so. More broadly, we also consider the role of misspecification in model selection.

Secondly, we analyze and discuss the standard conventions in Bayesian estimation used for the selection of priors. (a) Pre-filtering the data before estimating the model, in order to investigate primarily the business cycle features of the data. Ferroni (2011) offers an interesting discussion on pre-filtering the data before

\footnotetext{
${ }^{5}$ Calibration can be viewed as selecting a degenerate prior with mass one at a given point.
} 
estimating a DSGE model vs. estimating the trend and trend-deviations simultaneously. (b) The choice of a prior density does not mean that any distribution would do. A normal density might not be used for variances because those cannot take on negative values. Theoretical restrictions on the range of values that a parameter can take must be factored accordingly in the selection of a prior. (c) Transforming a parameter to change its parameter space is a convenient way to implement certain prior distributions. (d) The Bayesian estimation procedure lends itself to recursive estimation - as long as the distributions that we characterize describe a well-behaved joint distribution - so we also consider the possibility of recursively selecting priors for subsets of parameters as a way to articulate the estimation.

In terms of the second question, we tackle the issue of selecting the model's observables. First, we discuss how the number of parameters limits the number of observables that we can use for Bayesian estimation. We also discuss the effect that this has on the VAR representation of a dynamic stochastic general equilibrium model - especially if the number of shocks is less than the number of core equations of the model. Moreover, we also point out that identification problems (and invertibility problems) can affect our ability to identify shocks, parameters and unobserved variables - for a related point see Carlstrom et al. (2009) on the identification of monetary shocks in a (closed-economy) New Keynesian model.

We also explore the precision of the estimation of open-economy models when measurement errors abound and data availability is short even for some advanced countries. Furthermore, the difference in methodologies across countries may also further complicate the task of the researcher. In particular, we seek to understand if certain trade variables could be effectively used in estimation to exploit the information content that they reveal about difficult-to-measure foreign variables. This last result implied by the model of Martínez-García and Wynne (2010) ties in with an older literature that includes variables like import and commodity prices on the right-hand side of Phillips curve regressions. We assess whether the same precision can be achieved by employing the simulated data generated from the calibrated version of the model and testing for the estimation efficiency under different sets of observable variables. We provide evidence that some measures of foreign trade might be useful when output and inflation data from the rest of the world are problematic, but may also lead to different estimation results.

\section{The Two-Country Model}

We postulate a two-country dynamic stochastic general equilibrium (DSGE) model with complete asset markets and nominal rigidities, subject to country-specific productivity and monetary shocks, as in MartínezGarcía and Wynne (2010). The framework is similar to that of Clarida et al. (2002) in its representation of the standard workhorse New Open Economy Macro (NOEM) model. Its building blocks are summarized in the Appendix, but a more detailed derivation of this model can be found in Martínez-García (2008).

We abstract from capital accumulation - considering only linear-in-labor technologies - and trend growth. We also adopt a cashless economy assumption, where money plays the sole role of unit of account. ${ }^{6}$ These simplification generate a very stylized economic environment, but are not essential for our investigation. We are concerned primarily with the identification of structural monetary shocks and of the key structural parameters that affect the (domestic and international) propagation mechanism for these shocks. For that, the workhorse NOEM model of Martínez-García (2008) provides us with a tractable environment that breaks

\footnotetext{
${ }^{6}$ For further discussion of the cashless economy assumption, see chapter 2 in Woodford (2003).
} 
monetary non-neutrality and permits the international transmission of shocks.

The NOEM model features two standard distortions in the goods markets - monopolistic competition in production and price-setting subject to Calvo (1983) contracts - but of those two the key assumption on which monetary non-neutrality hinges is price stickiness. Openness to trade in goods, supported with international borrowing and lending under complete international asset markets, is the endogenous channel through which monetary shocks get transmitted. ${ }^{7}$ We cancel out the distortion caused by a market structure that is not perfectly competitive. As before, the added complexity of the model is not essential for our investigation of identification in Bayesian estimation.

Pass-through is complete and the law of one price holds at the variety level (even under price stickiness) by assuming that prices are set always in the producer's own currency. Deviations from purchasing-power parity (PPP) still arise from the assumption of home-product bias in consumption - and their magnitude depends on the degree of openness in both countries. Nonetheless, movements in real exchange rates and terms of trade do not cause a misallocation of demand across countries. ${ }^{8}$ Monopolistic competition in final goods markets also introduces a distortion in the allocation of resources (labor) within a country, but we correct for that by introducing an optimal labor subsidy for firms funded with non-distortionary, lump-sum taxes raised from the local households. Under flexible prices, that labor subsidy suffices to restore the perfectly competitive factor allocation.

Finally, price stickiness breaks monetary policy neutrality in the short-run - establishing a Phillips curve relationship between domestic inflation and global slack that monetary policy exploits - but preserves it in the long-run. Absent nominal rigidities (which would happen under flexible prices), monetary policy has no real effects neither in the long-run (steady state) nor the short-run (dynamics). The presence of this friction is fundamental to explain the dynamics of the model, and also crucial to identify monetary shocks and their propagation mechanism. Understanding this channel for monetary non-neutrality and the resulting real effects is, naturally, of great importance for monetary policy as it would determine the reach and trade-offs of monetary policy itself.

\subsection{The NOEM Model Dynamics}

We summarize the log-linearized equilibrium conditions of the model that we use to characterize the solution (locally) in Tables 1 and 2. We denote $\widehat{g}_{t} \equiv \ln G_{t}-\ln \bar{G}$ the deviation of a variable in logs from its steadystate. We define a vector of endogenous variables, $\widehat{Y}_{t}$, and a vector of exogenous (state) variables, $\widehat{X}_{t}$. The vector of endogenous variables can be split in a vector of endogenous core (or state) variables, $\widehat{Y}_{t}^{c}$, and a vector of non-core variables, $\widehat{Y}_{t}^{n}$. In our model, the endogenous core variables $\widehat{\pi}_{t}$ and $\widehat{\pi}_{t}^{*}$ denote Home and Foreign inflation (quarter-over-quarter changes in the consumption-based price index), $\widehat{x}_{t}$ and $\widehat{x}_{t}^{*}$ define the Home and Foreign output gaps (deviations of output from its potential in a frictionless environment), while $\widehat{i}_{t}$ and $\hat{i}_{t}^{*}$ are the Home and Foreign short-term nominal interest rates set by the policy-makers for the conduct of monetary policy.

\footnotetext{
${ }^{7}$ Another often-cited distortion in the goods markets that can affect the size of the real effects from monetary shocks across countries comes from international pricing behavior that results in incomplete exchange rate pass-through. However, for clarity of exposition we do not incorporate this feature to emphasize that identification of monetary shocks and the structural parameters that determine their propagation can be non-trivial even in the presence of the simplest possible departure from flexible prices.

${ }^{8}$ For details on the role of international price-setting in the missallocation of demand across countries and the design of optimal monetary policy, see also Engel (2009).
} 
The non-core endogenous variables of the model include aggregate output, $\widehat{y}_{t}$ and $\widehat{y}_{t}^{*}$, aggregate consumption, $\widehat{c}_{t}$ and $\widehat{c}_{t}^{*}$, aggregate employment, $\widehat{l}_{t}$ and $\widehat{l}_{t}^{*}$, and real wages, $\widehat{w}_{t}-\widehat{p}_{t}$ and $\widehat{w}_{t}^{*}-\widehat{p}_{t}^{*}$, in both countries. We also derive expressions for the terms of trade, $\widehat{t o t}_{t}$, the real exchange rate, $\widehat{r s}_{t}$, real exports and real imports, $\widehat{\exp }_{t}$ and $\widehat{i m p}_{t}$, and the real trade balance, $\widehat{t b}_{t} \cdot{ }^{9}$ There are two types of country-specific, exogenous shocks in the model: productivity shocks, $\widehat{a}_{t}$ and $\widehat{a}_{t}^{*}$, and monetary shocks, $\widehat{m}_{t}$ and $\widehat{m}_{t}^{*}$. We model the productivity shocks and the monetary shocks as two $\operatorname{VAR}(1)$ stochastic processes, but we only incorporate cross-country spillovers in the stochastic process for productivity shocks (not for monetary shocks). Productivity innovations and monetary innovations can be correlated across countries, but not with each other.

As shown in Table 1, the log-linearized core equilibrium conditions can be described with an openeconomy Phillips curve, an open-economy investment-savings (IS) equation and a Taylor rule for monetary policy in each country. ${ }^{10}$ The dynamics of the core endogenous variables can be cast in a canonical linear expectational difference model under rational expections, i.e.,

$$
A \mathbb{E}_{t}\left[\widehat{Y}_{t+1}^{c}\right]=B \widehat{Y}_{t}^{c}+C \widehat{X}_{t}
$$

where the matrices $A, B$, and $C$ are expressed in terms of the structural parameters of the model. As shown in Table 2, the log-linearized conditions for the non-core endogenous variables can be expressed as a linear transformation of the endogeneous core variables and the exogenous shocks, i.e.,

$$
\widehat{Y}_{t}^{n}=D \widehat{Y}_{t}^{c}+F \widehat{X}_{t}
$$

where the matrices $D$ and $F$ are expressed in terms of a subset of the structural parameters of the model. Finally, we specify a driving process for the exogenous variables - reported in Table 1 - of the form,

$$
\widehat{X}_{t}=\rho \widehat{X}_{t-1}+\widehat{\varepsilon}_{t}, \mathbb{E}_{t}\left[\widehat{\varepsilon}_{t} \widehat{\varepsilon}_{t}^{T}\right]=\Omega
$$

where $\rho$ governs the dynamics and $\Omega$ the variances-covariances of the exogenous (state) variables of the model.

\section{[Insert Table 1 about here.] \\ [Insert Table 2 about here.]}

The core structure of the model descibed in Table 1 incorporates an open-economy Phillips curve. This equilibrium condition fleshes out the global slack hypothesis - that is, the idea that in a world open to

\footnotetext{
${ }^{9}$ In a two-country model, suffices to determine the trade patterns from the point of view of the Home country only, as we do here.

${ }^{10}$ The core of the model refers to a (minimal) set of equations that uniquely determines the path of a subset of endogenous variables (the core or state variables) by their initial conditions and the path of the exogenous shocks specified. In turn, all non-core (or non-state) variables can be expressed as functions of the core endogenous variables and the specified exogenous shocks. The core system of equations, therefore, suffices to determine uniquely the future paths of all the core and non-core endogenous variables. Often there is no unique way of characterizing the core and solving the model. Moreover, for Bayesian estimation purposes the number of observables and - therefore the number of estimating equations (core or noncore) - is bound to the number of shocks to be estimated from the data. Hence, the core equations may need to be complemented with non-core equations for estimation purposes (e.g., if exogenous labor supply shocks and government consumption shocks in each country were added to our model).
} 
trade and under short-run monetary non-neutrality (arising from nominal rigidities in the goods market modelled as price stickiness), the relevant trade-off for monetary policy is between domestic inflation and global (rather than local) slack. Martínez-García and Wynne (2010) provides some further discussion of the open-economy Phillips curve and describes its extension under alternative assumptions on international price-setting behavior of firms.

The open-economy IS equation illustrates how the output gaps - output deviations from potential, where potential output is defined as the output that would prevail in a frictionless environment under the same shock realization and denoted as $\widehat{\bar{y}}_{t}$ and $\widehat{\bar{y}}_{t}^{*}$ in the Home and Foreign country respectively - are tied to both Home and Foreign demand forces. The Fisher equation for real interest rates in the Home and Foreign country defines them as $\widehat{r}_{t} \equiv \widehat{i}_{t}-\mathbb{E}_{t}\left[\widehat{\pi}_{t+1}\right]$ and $\widehat{r}_{t}^{*} \equiv \widehat{i}_{t}^{*}-\mathbb{E}_{t}\left[\widehat{\pi}_{t+1}^{*}\right]$ respectively. Nominal rigidities à la Calvo (1983) introduce an intertemporal wedge between the actual real interest rate (the opportunity cost of consumption today versus consumption tomorrow) and the natural (real) rate of interest that would prevail in a frictionless equilibrium. The natural (real) rates of interest are denoted $\widehat{\bar{r}}_{t}$ for the Home country and $\widehat{\bar{r}}_{t}^{*}$ for the Foreign country, and are invariant to monetary policy or the monetary policy shocks. Demand itself responds to deviations of each country's real interest rate from its natural real rate as that would shift consumption demand across time.

Whenever the real interest rate is above its natural real rate, more consumption today is being postponed for consumption tomorrow than would be the case in the frictionless environment. Ceteris paribus, that implies a demand shortfall today (a decline in the output gap or a fall in output relative to potential) and the expectation of that slack unwinding in the future. Analogously, when the real interest rate is below the natural rate, the resulting boost in consumption today (at the expense of future consumption) leads to a temporary increase in the output gap that is expected to dissipate over time. The open-economy IS equation illustrates that demand for local goods can be either domestic or foreign (the latter resulting in exports), so real interest rate deviations in both countries matter.

The Home and Foreign monetary policy rules complete the specification of the core model. Those rules reflect the conventional view that monetary policy still pursues the goal of domestic stabilization (even in a fully integrated world) and, hence, solely responds to changes in domestic economic conditions. Monetary policy is modelled with a Taylor (1993)-type rule and is assumed to react to local conditions as determined by each country's inflation and output gap alone. ${ }^{112}$ To be consistent with the simple rule laid out in Taylor (1993), we assume that the persistence in policy rates can be thought of as extrinsic or exogenous inertia in the policy-making process (and out of the policy-makers control). Extrinsic persistence could result from imperfections such as the slow acquisition of information relevant for setting monetary policy. In contrast, intrinsic or endogenous inertia results from policy-makers intentionally smoothing out their policy response to changing economic conditions (see, e.g., Rudebusch (2002) and Rudebusch (2006) for further discussion). ${ }^{13}$

\footnotetext{
${ }^{11}$ The frictionless allocation would be attained if monetary policy-makers in both countries aggressively target local inflation alone to ensure that $\widehat{\pi}_{t}=0$ and $\widehat{\pi}_{t}^{*}=0$ for all $t$. That would be a limiting case of the Taylor rule posited here when the response to inflation deviations becomes arbitrarily large.

${ }^{12}$ The Taylor rule specification in Table 1 is consistent with different institutional arrangements. For example, it would be consistent with a policy framework where the central bank operates under a dual mandate (the Fed) or under a single mandate on price stability (the ECB), depending on how we parameterize the monetary policy's reaction to inflation and the output gap in each case.

${ }^{13}$ The distinction between extrinsic and intrinsic inertia is of great importance for policy evaluation, as it changes the dynamics of the model and the transmission mechanism of shocks. Uncertainty about the source of inertia in monetary policy is one of the key problems related to misspecification and model selection that we investigate in the context of implementing
} 
A common problem in estimating many standard (open- or closed-economy) New Keynesian models is that the subset of core endogenous variables includes Home and Foreign output gaps that are not directly observable - as only output can be directly measured in the data, but not potential output which is model dependent. Moreover, in our model, the other two core variables (inflation and nominal interest rates) are nominal - not real - while conventional practice suggests that it would be "best" to include both nominal and real variables in the estimation in order to test the real-nominal trade-off implied by monetary non-neutrality (the Phillips curve). One conventional approach often used in applied work to deal with unobservable output gaps consists in replacing the output gap with a statistical proxy derived from observable data in reducedform regressions (e.g., Martínez-García and Wynne (2010) investigate the usefulness of HP-filtered output as a proxy for the output gap). Another alternative consists in adopting a more structural approach to estimation making use of the constraints implied by theory itself for identification. In that case, which is more relevant for our paper, an observation equation for each country relating the output gap to other observables (e.g., current ouput or other macro and trade aggregates) and a model-consistent specification of the output potential must be added to the core model, hence jointly estimating the core model and the specification of output potential. The selection of observables in this context and its influence on identification are another area of interest for us. Moreover, the endogenous non-core variables impose theoretical constraints on the data that can also be exploited to estimate the model and to facilitate the identification of certain key structural parameters. That is another aspect of the "art" of estimation that we attempt to highlight in this paper.

\subsection{The Frictionless Model Dynamics}

Table 3 describes the full dynamics of the economy in a frictionless environment under flexible prices and perfect competition. We distinguish variables in the frictionless equilibrium by marking them with an upper bar. In the frictionless model, we let $\widehat{\bar{g}}_{t} \equiv \ln \bar{G}_{t}-\ln \bar{G}$ denote the deviation of an endogenous variable in logs from its steady-state value. The exogenous monetary and productivity shocks are, however, invariant to the specification of the model - the frictionless and the NOEM models are subject to the same realization of these shocks. All endogenous variables described before have a natural counterpart in the frictionless equilibrium except for the output gaps because by definition current and potential output must be the same in a model without frictions.

Inflation dynamics are determined by the monetary policy rule and are sensitive to both productivity and monetary shocks. In the limit as the response to inflation deviations becames arbitrarily large, inflation tends to zero. As expected under monetary neutrality, it follows from the characterization of the dynamics of the frictionless model that neither the monetary policy rule nor monetary shocks have an impact on any real variables (i.e., on (potential) output, consumption, employment, real wages and the (natural) real interest rates).

The natural (real) rates of interest in the frictionless model, $\widehat{\bar{r}}_{t}$ and $\widehat{\bar{r}}_{t}^{*}$, can be expressed as a function of expected changes in Home and Foreign potential output, reflecting the fact that real rates respond to expected changes in real economic activity - as measured by output - rather than to the overall level of economic activity. We characterize potential output for each country, $\widehat{\bar{y}}_{t}$ and $\widehat{\bar{y}}_{t}^{*}$, as a function of the Home

Bayesian estimation. This distinction is not trivial because the dynamics of this simple model would be different. In the presence of intrinsec inertia, the model incorporates a backward-looking element (a state variable) that is otherwise not present in the current specification with extrinsic inertia. 
and Foreign productivity shocks. As a result, the real interest rates in a frictionless economy (natural rates) are a function of productivity shocks alone and invariant to the specification of the monetary policy rule or to monetary policy shocks. The natural real interest rate is affected by the dynamics of productivity in the Home and Foreign countries due to openness to trade, but the rates do not equalize across countries in spite of the symmetry embedded in the model because we have assumed home-product bias in consumption. Homeproduct bias, in turn, translates into different consumption baskets for the Home and Foreign countries, ${ }^{14}$ and differences in the consumption baskets imply that each country's consumption demand responds differently to a given country-specific shock (resulting in different natural rates of interest across countries).

\section{[Insert Table 3 about here.]}

As a convenient first step for the estimation of the NOEM model, we derive a characterization of the dynamics of potential output and the natural rates of interest based on the frictionless model described in Table 3. The estimation of the NOEM model could be attempted by assuming a reduced form representation for potential output and the natural rates, but it would not constitute a truly structural approach to estimation as we would be ignoring constraints implied by the thoery on the dynamics of these variables. Here we adopt a fully structural approach and derive an analytic solution to both, so that when we estimate jointly the NOEM model and its frictionless counterpart we do it consistently. Hence, in that spirit, the following proposition gives us a characterization of potential output for both countries derived from the stochastic $\operatorname{VAR}(1)$ process for the productivity shocks in the following terms,

Proposition 1 The potential output of the Home and Foreign countries $\widehat{\bar{y}}_{t}$ and $\widehat{\widehat{y}}_{t}^{*}$ described in Table 3 can be defined as a linear transformation of the Home and Foreign productivity shocks $\widehat{a}_{t}$ and $\widehat{a}_{t}^{*}$, i.e.,

$$
\left(\begin{array}{c}
\widehat{\bar{y}}_{t} \\
\widehat{\bar{y}}_{t}^{*}
\end{array}\right) \approx\left(\frac{1+\varphi}{\gamma+\varphi}\right)\left(\begin{array}{cc}
\Lambda & (1-\Lambda) \\
(1-\Lambda) & \Lambda
\end{array}\right)\left(\begin{array}{c}
\widehat{a}_{t} \\
\widehat{a}_{t}^{*}
\end{array}\right) .
$$

Given the VAR(1) structure assumed for the productivity shocks, we can derive the following VAR(1) stochastic process to characterize the dynamics of potential output,

$$
\begin{aligned}
\left(\begin{array}{c}
\widehat{\bar{y}}_{t} \\
\widehat{\bar{y}}_{t}^{*}
\end{array}\right) & \approx\left(\begin{array}{cc}
\delta_{a} & \delta_{a, a^{*}} \\
\delta_{a, a^{*}} & \delta_{a}
\end{array}\right)\left(\begin{array}{c}
\widehat{\bar{y}}_{t-1} \\
\widehat{\bar{y}}_{t-1}^{*}
\end{array}\right)+\left(\begin{array}{c}
\widehat{\varepsilon}_{t}^{y} \\
\widehat{\varepsilon}_{t}^{y *}
\end{array}\right), \\
\left(\begin{array}{c}
\widehat{\varepsilon}_{t}^{y} \\
\widehat{\varepsilon}_{t}^{y *}
\end{array}\right) & \sim N\left(\left(\begin{array}{l}
0 \\
0
\end{array}\right), \sigma_{y}^{2}\left(\begin{array}{cc}
1 & \rho_{y, y^{*}} \\
\rho_{y, y^{*}} & 1
\end{array}\right)\right),
\end{aligned}
$$

where we define the volatility and the correlation of the potential output innovations in the following fashion,

$$
\begin{aligned}
\sigma_{y}^{2} & =\sigma_{a}^{2}\left(\frac{1+\varphi}{\gamma+\varphi}\right)^{2}\left((\Lambda)^{2}+2 \rho_{a, a^{*}} \Lambda(1-\Lambda)+(1-\Lambda)^{2}\right), \\
\rho_{y, y^{*}} & =\frac{\rho_{a, a^{*}}(\Lambda)^{2}+2 \Lambda(1-\Lambda)+\rho_{a, a^{*}}(1-\Lambda)^{2}}{(\Lambda)^{2}+2 \rho_{a, a^{*}} \Lambda(1-\Lambda)+(1-\Lambda)^{2}} .
\end{aligned}
$$

\footnotetext{
${ }^{14}$ That is the case except in a knife-edge situation where both Home and Foreign households' share of domestic and imported goods coincides with the share of locally-produced goods. In the context of our model, there is a mass one of varieties equally split across countries, so the share of locally-produced goods in the consumption basket of each country would have to be $\frac{1}{2}$.
} 
Proof. See the Appendix.

The following corollary gives us a simple characterization of the natural rates in the Home and Foreign countries $\widehat{\bar{r}}_{t}$ and $\widehat{r}_{t}^{*}$ derived also from the stochastic $\operatorname{VAR}(1)$ process for the productivity shocks in the following terms,

Corollary 1 The natural rates of the Home and Foreign countries $\widehat{\bar{r}}_{t}$ and $\widehat{\bar{r}}_{t}^{*}$ described in Table 3 can be defined as a linear transformation of the Home and Foreign productivity shocks $\widehat{a}_{t}$ and $\widehat{a}_{t}^{*}$, i.e.,

$$
\begin{aligned}
& \left(\begin{array}{c}
\widehat{r}_{t} \\
\widehat{r}_{t}^{*}
\end{array}\right) \approx \gamma\left(\frac{1+\varphi}{\gamma+\varphi}\right)\left(\begin{array}{cc}
(\Theta \Lambda+(1-\Theta)(1-\Lambda)) & (1-(\Theta \Lambda+(1-\Theta)(1-\Lambda))) \\
(1-(\Theta \Lambda+(1-\Theta)(1-\Lambda))) & (\Theta \Lambda+(1-\Theta)(1-\Lambda))
\end{array}\right)\left(\begin{array}{c}
\mathbb{E}_{t}\left[\Delta \widehat{a}_{t+1}\right] \\
\mathbb{E}_{t}\left[\Delta \widehat{a}_{t+1}^{*}\right]
\end{array}\right) \\
& \approx \gamma\left(\frac{1+\varphi}{\gamma+\varphi}\right)\left(\begin{array}{cc}
(\Theta \Lambda+(1-\Theta)(1-\Lambda)) & (1-(\Theta \Lambda+(1-\Theta)(1-\Lambda))) \\
(1-(\Theta \Lambda+(1-\Theta)(1-\Lambda))) & (\Theta \Lambda+(1-\Theta)(1-\Lambda))
\end{array}\right)\left(\begin{array}{cc}
\delta_{a}-1 & \delta_{a, a^{*}} \\
\delta_{a, a^{*}} & \delta_{a}-1
\end{array}\right)\left(\begin{array}{c}
\widehat{a}_{t} \\
\widehat{a}_{t}^{*}
\end{array}\right) .
\end{aligned}
$$

Given the VAR(1) structure assumed for the productivity shocks, we can derive the following VAR(1) stochastic process to characterize the dynamics of the natural rates,

$$
\begin{aligned}
\left(\begin{array}{c}
\widehat{\bar{r}}_{t} \\
\widehat{\bar{r}}_{t}^{*}
\end{array}\right) & \approx\left(\begin{array}{cc}
\delta_{a} & \delta_{a, a^{*}} \\
\delta_{a, a^{*}} & \delta_{a}
\end{array}\right)\left(\begin{array}{c}
\widehat{\bar{r}}_{t-1} \\
\widehat{\bar{r}}_{t-1}^{*}
\end{array}\right)+\left(\begin{array}{c}
\widehat{\varepsilon}_{t}^{r} \\
\widehat{\varepsilon}_{t}^{r *}
\end{array}\right), \\
\left(\begin{array}{c}
\widehat{\varepsilon}_{t}^{r} \\
\widehat{\varepsilon}_{t}^{r *}
\end{array}\right) & \sim N\left(\left(\begin{array}{c}
0 \\
0
\end{array}\right), \sigma_{r}^{2}\left(\begin{array}{cc}
1 & \rho_{r, r^{*}} \\
\rho_{r, r^{*}} & 1
\end{array}\right)\right),
\end{aligned}
$$

where we define the volatility and the correlation of the natural rate innovations in the following way,

$$
\begin{aligned}
\sigma_{r}^{2} & =\sigma_{a}^{2} \gamma^{2}\left(\frac{1+\varphi}{\gamma+\varphi}\right)^{2}\left(\left(\Pi_{1}\right)^{2}+2 \rho_{a, a^{*}} \Pi_{1} \Pi_{2}+\left(\Pi_{2}\right)^{2}\right), \\
\rho_{r, r^{*}} & =\frac{\rho_{a, a^{*}}\left(\Pi_{1}\right)^{2}+2 \Pi_{1} \Pi_{2}+\rho_{a, a^{*}}\left(\Pi_{2}\right)^{2}}{\left(\Pi_{1}\right)^{2}+2 \rho_{a, a^{*}} \Pi_{1} \Pi_{2}+\left(\Pi_{2}\right)^{2}}
\end{aligned}
$$

and,

$$
\begin{aligned}
\Pi_{1} & \equiv(\Theta \Lambda+(1-\Theta)(1-\Lambda))\left(\delta_{a}-1\right)+(1-(\Theta \Lambda+(1-\Theta)(1-\Lambda))) \delta_{a, a^{*}} \\
& =\delta_{a, a^{*}}-\xi\left(\frac{\varphi(\sigma \gamma-(\sigma \gamma-1)(2 \xi-1))+\gamma}{\varphi\left(\sigma \gamma-(\sigma \gamma-1)(2 \xi-1)^{2}\right)+\gamma}\right)\left(1+\delta_{a, a^{*}}-\delta_{a}\right), \\
\Pi_{2} & \equiv(\Theta \Lambda+(1-\Theta)(1-\Lambda)) \delta_{a, a^{*}}+(1-(\Theta \Lambda+(1-\Theta)(1-\Lambda)))\left(\delta_{a}-1\right) \\
& =\left(\delta_{a}-1\right)+\xi\left(\frac{\varphi(\sigma \gamma-(\sigma \gamma-1)(2 \xi-1))+\gamma}{\varphi\left(\sigma \gamma-(\sigma \gamma-1)(2 \xi-1)^{2}\right)+\gamma}\right)\left(1+\delta_{a, a^{*}}-\delta_{a}\right) .
\end{aligned}
$$

Proof. See the Appendix.

We note that the productivity shocks enter into the dynamics described in Table 1 only through their impact on the dynamics of the natural real rates in this economy $\widehat{\bar{r}}_{t}$ and $\widehat{\bar{r}}_{t}^{*}$. Having established the solution to the natural rates in the frictionless model in Corollary 1 allows us to simplify the presentation of the NOEM model, as we adopt the specification of the natural rates and monetary shocks as our forcing processes from now on. The Home and Foreign monetary shock processes $\widehat{m}_{t}$ and $\widehat{m}_{t}^{*}$ do not require transformation as they 
enter directly into the model through the specification of the Taylor rule for monetary policy in each country.

Home and Foreign potential output - as well as the Home and Foreign natural rates - inherit the VAR(1) stochastic structure of the productivity shocks and, moreover, some of the basic features of the underlying productivity shocks - in particular, their persistence and spillovers. This is an interesting finding in its own right because it illustrates the oft-made claim that endogenous persistence is inherited from the assumed persistence of productivity shocks. That certainly is true for potential output and for the natural rates in our frictionless model. However, Proposition 1 and Corollary 1 also indicate that - unlike what happens with persistence and spillovers - the matrix of variances-covariances (both the volatility and the correlation) of the potential output and natural rate processes is different from the one posited for the exogenous productivity shocks. The structural parameters of the model are going to modify the volatility and correlations, so we would not be identifying structural productivity shocks from the model if we were to postulate a reduced-form $\operatorname{VAR}(1)$ for the output potential and natural rate processes. Moreover, if we were to ignore the restrictions implied by theory on the matrix of variances-covariances, we would be ignoring relevant cross-equation restrictions that can be exploited to identify the structural parameters of the model (not only the volatility and correlation of the productivity shocks).

In this case, the linear rational expectations frictionless model that completes the characterization of the core endogenous variables reduces to a simple two-equation system for Home and Foreign inflation $\widehat{\bar{\pi}}_{t}$ and $\widehat{\bar{\pi}}_{t}^{*}$, described in Table 3 as,

$$
\begin{aligned}
& \mathbb{E}_{t}\left[\widehat{\bar{\pi}}_{t+1}\right] \approx \psi_{\pi} \widehat{\bar{\pi}}_{t}+\widehat{m}_{t}-\widehat{\bar{r}}_{t}, \\
& \mathbb{E}_{t}\left[\widehat{\bar{\pi}}_{t+1}^{*}\right] \approx \psi_{\pi} \hat{\bar{\pi}}_{t}^{*}+\widehat{m}_{t}^{*}-\widehat{\bar{r}}_{t}^{*},
\end{aligned}
$$

where $\widehat{\bar{r}}_{t}$ and $\widehat{\bar{r}}_{t}^{*}$ denote the Home and Foreign natural rates and $\widehat{m}_{t}$ and $\widehat{m}_{t}^{*}$ are the Home and Foreign monetary shocks. Naturally, monetary shocks only have an effect on nominal variables in the frictionless model, as monetary neutrality holds in the short-run as well as in the long-run. Moreover, we find that whenever $\psi_{\pi}$ converges towards infinity, the solution implies zero-inflation in both countries. That is the costumary simplification embedded in most real business cycle (RBC) models. In our context, it happens to be also the optimal monetary policy for the NOEM model that we have postulated here, as zero inflation prevents the relative price dispersion that results from firms adjusting their prices in response to changes in the price level at different times - and relative price dispersion is at the heart of the distortion arising from price stickiness.

Finally, the deterministic steady state of the model is presented in Table 4. The steady state is identical for the NOEM and frictionless models, reflecting the fact that monetary neutrality holds in the long-run. Monetary policy has no direct impact on real variables in steady state, so long-run neutrality is preserved even though nominal rigidities in the NOEM model introduce a trade-off between local inflation and global output gap in the short-run dynamics.

[Insert Table 4 about here.] 


\section{Calibration and Simulation}

Both countries are of equal size in terms of household population and the share of varieties they produce. We also assume that the deep structural parameters governing tastes (and technologies) are the same in the two countries. The parameters governing the degree of price stickiness (that is, the goods market friction) and the home-product bias (that is, the degree of openness) are common in the two countries, and the monetary policy regime is characterized by an identical Taylor rule in both economies. The productivity and monetary stochastic processes are modelled as symmetric as well. Moreover, the imposition of an optimal labor subsidy, $\phi$, to compensate for the mark-up distortion introduced by monopolistic competition means that the subsidy itself and the elasticity of substitution across varieties, $\theta$, drop entirely from the log-linearized rational expectations NOEM model (with or without the goods market friction) and from its steady state. ${ }^{15}$

Yet, even with the inherent symmetry of both countries and the simplification arising from the adoption of an optimal labor subsidy, the long-run (steady state) and short-run (dynamics) of the model still depend on a large number of parameters - 18 in total. Up to 15 of those parameters affect the dynamics of the model depending on whether the goods market friction is present or not. The remaining 3 model parameters affect solely the long-run features of the model but not the endogenous short-run propagation, and 2 of them that are unrestricted by the steady state itself would be subject to a normalization. There are 2 other model parameters entering into the steady state that also affect the short-run dynamics, and those can be pinned down irrespectively of how we tackle the steady state normalization. Those 15 model parameters that influence the short-run dynamics need to be calibrated in order to simulate data, as we subsequently attempt to recover them with recourse to Bayesian estimation techniques.

We split the full set of 18 model parameters between 9 shock parameters that characterize the exogenous shock processes ( 7 of which affect the exogenous dynamics of the shocks) and 9 structural parameters that are related to preferences, nominal frictions and policy ( 8 of which affect the endogenous propagation of the shocks). In turn, we distinguish the structural parameters between those that enter into the characterization of the deterministic steady state ( 3 of them, but one subject to normalization) and those that influence the endogenous propagation of shocks within the model ( 8 of them). The intersection is a non-empty set, as there are 2 structural parameters that affect both the steady state as well as the dynamics of the model. We list all of the parameters of the model and summarize the role of each structural and shock parameter in the steady state and the dynamics of the model in Table 5 - as this information is relevant both to calibrate the model and to elicit proper priors.

\section{[Insert Table 5 about here.]}

But, which model parameters are really crucial for us to identify and recover precisely in this openeconomy, monetary model? The answer to this question has to be tied to the fundamental question(s) that the model is designed to address. The aims of the model that we are investigating are two-fold and well-defined in the international macro literature: (a) to quantify the real effects of monetary non-neutrality (which depend on the assumption of nominal rigidities as summarized by the structural parameter $\alpha$ ), and (b)

\footnotetext{
${ }^{15}$ Monopolistic competition introduces a mark-up over marginal costs that is a function of the elasticity of substitution across varieties within a country, $\theta$. The mark-up is the only place where the parameter $\theta$ shows up in the model up to a first-order approximation, so the optimal labor subsidy $\phi$ which neutralizes the mark-up distortion also makes the pair $(\phi, \theta)$ irrelevant for the characterization of the steady state and the dynamics of the model.
} 
to quantify the strength of the international transmission mechanism of shocks (which for monetary shocks requires also monetary non-neutrality, but more generally it depends on the degree of openness of these economies as given by the parameter $\xi$ ). Obviously, the proper identification of the structural parameters $\alpha$ and $\xi$ seems crucial given the central role they play in the model set-up. The structural parameters of interest, however, are not limited to $\alpha$ and $\xi$ alone. Consider the following ${ }^{16}$ :

- The sub-optimality of the monetary policy regime specified by the policy parameters $\psi_{\pi}$ and $\psi_{x}$.

Approximating the optimal monetary policy in this model requires us to set $\psi_{\pi}$ arbitrarily large to ensure that $\widehat{\pi}_{t}$ and $\widehat{\pi}_{t}^{*}$ are arbitrarily close to zero and that the paths of $\widehat{y}_{t}$ and $\widehat{y}_{t}^{*}$ are arbitrarily close to their respective potential $\widehat{\bar{y}}_{t}$ and $\widehat{\bar{y}}_{t}^{*}$. Under an optimal monetary policy rule in both countries, we will not find evidence of real effects from monetary policy shocks or evidence that Foreign monetary shocks could have an effect on the domestic macro aggregates irrespective of the degree of price stickiness $\alpha$ and openness $\xi$ that characterizes both economies. Hence, how "far" the prevailing monetary policy regime is from that first-best monetary policy rule is crucial to detecting (and quantifying) the real effects and the propagation channels implied by the nominal rigidity and openness.

- The responsiveness of demand regulated by the inverse of the Frisch elasticity of labor supply, $\varphi$, and the inverse of the intertemporal elasticity of substitution, $\gamma$.

These preference parameters enter into the calculations of the slope of the Phillips curve with respect to the output gaps and, therefore, have a measurable impact on the real effects arising from monetary non-neutrality (see Table 1). These two parameters affect the sensitivity of marginal costs (real wages) to demand-driven increases in production and employment as well as the consumption demand (in response to shocks). Their impact cannot be ignored as they directly influence the trade-offs that policy-makers face between nominal and real variables.

- The risk-sharing role of the terms of trade implied by the elasticity of substitution between Home and Foreign bundles, $\sigma$.

Irrespective of the structure of financial markets, it is well-known that full insulation from foreign productivity shocks can be attained in the frictionless model through movements of the terms of trade alone whenever $\sigma \gamma=1$. The sign of the effect of a foreign productivity shock on domestic output would depend on whether $\sigma \gamma$ is greater or smaller than one. Moreover, monetary non-neutrality implies that foreign monetary shocks have no real effects in any case. Similarly, the international transmission of shocks will be affected by the degree of substitutability between locally-produced and imported goods. In fact, not only the magnitude but the sign of the international propagation of the shocks in this model depends on our ability to correctly identify the parameters $\sigma$ and $\gamma$.

\section{[Insert Table 6 and Figure 1 about here.]}

We proceed now by describing the strategy to calibrate all the structural parameters that we have encountered in the model, so that we can turn it into our DGP process. ${ }^{17}$ In eliciting appropriate priors for

\footnotetext{
${ }^{16}$ The parameter $\xi$ affects the short-run dynamics of the model, but it can be pinned down in the steady state. The other structural parameter that enters both into the steady state and the dynamics of the model is $\beta$. In turn, $\alpha$ only affects the dynamics and, therefore, we cannot rely on long-run features of the data to set its parameterization. Similarly, all other structural parameters that cannot be identified through steady state relationships can still alter our interpretation/quantification of the model and are subject of considerable debate.

${ }^{17} \mathrm{An}$ important recommendation for calibration that we only treat in passing is to choose parameter values that would be
} 
Bayesian estimation, the range on which the structural parameters are defined is one of the first issues that researchers need to take into account. This, however, is very important as well in calibration as we want to set parameter values that are consistent with a well-behaved solution. We summarize our calibration and the range of parameter values that we invoke to guarantee the existence of a unique solution in Table 6 . Figure 1 describes the region of the policy parameter space for $\psi_{\pi}$ and $\psi_{x}$ where a unique solution exists (the necessary and sufficent conditions for determinacy of our model), given our calibration of all other non-policy parameters.

Parameters related to the steady state (long-run). Typically, long-run historical averages of the relevant macroeconomic time-series are used to calibrate steady state parameters. Given our model specification, there are at most 7 parameters (as indicated in Table 5) that enter into the steady state: the intertemporal discount factor, $\beta$, the inverse of the intertemporal elasticity of substitution, $\gamma$, the inverse of the Frisch elasticity of labor supply, $\varphi$, the labor disutility scaling factor, $\kappa$, the share of locally-produced goods, $\xi$, the unconditional mean of the productivity shocks, $A$, and the unconditional mean of the monetary shock, $M$. Of those 7 parameters, the unconditional mean of the monetary shock is pinned down by the steady state Taylor rule to be equal to one - although it does not affect the short-run dynamics. Only 4 of the remaining 6 parameters can be matched to long-run historical averages simultaneously based on the steady state relationships described in Table 4 .

A calibration strategy that can be easily applied in our model is to choose $A$ in order to match the longrun average of labor productivity (expressed in units of output per unit of employment or hours worked, $\left.\frac{\bar{Y}}{\bar{L}}\right)$. Then, given the set $A$ as well as the calibration imposed on the preference parameters $\gamma$ and $\varphi$, we would select the scaling factor $\kappa$ to match the long-run average of the level of output (or, alternatively, the level of consumption or employment). ${ }^{18}$ The preference parameters $\gamma$ and $\varphi$ would have to be pinned down in some other way. Notice that the parameters $A$ and $\kappa$ are two of the three parameters that characterize the steady state but that do not affect the dynamics of the model (the other one being the parameter $M$ ), so a normalization can be imposed on both without loss of generality - as it would not interfere with the endogenous propagation channels. For tractability, we simply normalize them such that $A=\kappa=1$ and obtain a steady state in which output, consumption and employment (in levels) for the Home and Foreign countries are all equal to one.

The preference parameters $\gamma$ and $\varphi$ cannot be pinned down by the steady state relationships, independently of how we parameterize/normalize $A$ and $\kappa$. In turn, the intertemporal discount factor, $\beta$, and the share of locally-produced goods, $\xi$, are two preference parameters that can be matched to historical macro-

consistent with the determinacy (existence and uniqueness) of the solution. The standard practice with linearized/log-linearized models is to calibrate the parameter values to satisfy the Blanchard-Kahn conditions for existence and uniqueness. For the model investigated here, this is not a major issue as the calibration in Table 5 is indeed consistent with the Blanchard-Kahn conditions ensuring that a unique solution exists. However, this might be an issue depending on the parameter values that we consider for the monetary policy rule and possibly under asymmetric policy rules and other alternative open-economy specifications. If a modified version of the Taylor principle - that is, the principle that central banks should increase interest rates more than one-for-one in response to higher inflation in order to "stabilize" the economy-is not satisfied, then the NOEM model presented here does not have a unique solution. Figure 1 illustrates the region of indeterminacy (multiple solutions) and determinacy (unique solution) for our model. Monetary policy can lead to indeterminacy even if only one country is thought to be in violation of the open-economy version of the Taylor principle. For further discussion of this point in a related New Keynesian model, see Bullard and Mitra (2007) and more specifically Bullard and Singh (2008).

${ }^{18}$ The model does not incorporate growth explicitly, so matching the long-run average of labor productivity and output level makes sense. However, most of these macroeconomic time series trend upwards in the data. Therefore, the parameterization strategy would need to be adjusted in the presence of (deterministic or stochastic) time trends. See Ferroni (2011) for a recent discussion on pre-filtering the data versus estimating the trend jointly with the rest of the model. 
economic time series based on steady state relationships. We set the intertemporal discount factor $\beta$ at 0.99 to attain an average yearly interest rate of $4 \%$ (i.e., we choose $\beta$ to imply that $\left(\frac{1}{\beta}\right)^{4}=1.041$ ). We set the share of locally-produced goods in the consumption basket $\xi$ at 0.94 in order to obtain an average import share of $6 \%$ for the U.S. These parametric choices are taken from Chari et al. (2002), which rely on U.S. and European data for their calibration. ${ }^{19}$

On the one hand, it is important to note that the parameters $M, A$ and $\kappa$ do not affect the dynamics of the NOEM model or the dynamics of its flexible price (frictionless) counterpart. Therefore, none of them has a direct impact on the propagation channels of the model that we aim to investigate. So we simply do not count these three parameters among those to be calibrated and estimated, reducing effectively the number of parameters of interest to the 15 other parameters that enter into the short-run dynamics of the NOEM model (indicated in Table 5). On the other hand, the preference parameters $\beta$ and $\xi$ that we pin down in steady state also affect the short-run dynamics of the model. The parameter $\beta$ affects the dynamics of the NOEM model (but not those of the frictionless model) as it enters into the Phillips curve specification and together with the degree of price stickiness (the Calvo parameter, $\alpha$ ) is a key determinant of its slope.

The parameter $\xi$ affects the short-run dynamics of the model - in this case, the NOEM model as well as the flexible price model-by regulating the degree of openness to trade. This parameter has only a role to play in the transmission of productivity shocks in the flexible price environment, as monetary neutrality implies there is no real effect in the transmission of monetary shocks. However, it affects the propagation of both productivity and monetary shocks in the NOEM model. In other words, the relative importance of foreign productivity and monetary shocks on domestic endogenous macro aggregates not only depends on the relevance of the friction (as measured by the parameter $\alpha$ ) but also on the degree of openness of these economies (as measured by $\xi$ ).

Identifying precisely both parameters $\beta$ and $\xi$ through steady state relationships is, therefore, of great empirical relevance and practical importance.

Parameters related to the model dynamics (short-run). Typically, it is not recommended to base the identification of the key endogenous propagation parameters on time series macro data that is directly linked to the questions being investigated in order to avoid an inherent circularity problem. In open-economy monetary models like the one we explore here where the central questions are to quantify the (short-run) monetary non-neutrality and the contribution of Home and Foreign monetary shocks over the business cycle, this prescription translates into the recommendation of not using aggregate output (or some measure of aggregate consumption or employment) and inflation as our primary data sources on which to base the identification of the parameters in the propagation mechanim. In our model, trade and international relative price data can play an important auxiliary role in calibrating some of those parameters.

It is also not recommended to use parameter estimates that are derived from theoretical constraints or relationships that are not compatible with (or easily mapped into) the structure of the model being proposed in order to avoid introducing a parameter bias in the calibration. Sometimes the structural parameters are identified from a pre-sample period of the time series macro aggregates that we want to explain with the aim of mitigating the circularity problem. ${ }^{20}$ It might even be possible to map some parameter estimates

\footnotetext{
${ }^{19}$ We assume that households in each country include the same share of locally-produced goods $\xi$ and imported goods (1 - $\xi$ ) in their respective consumption baskets. In contrast, Clarida et al. (2002) and Woodford (2010) - among others-make the assumption that both countries have idential consumption baskets.

${ }^{20}$ In practice, however, the pre-sample identification of the parameters should not differ greatly from those obtained in-sample
} 
obtained from similar models. Most often, however, the techniques preferred to identify the structural parameters affecting the short-run dynamics involve in-sample macro data (not primarily of the same series we are interested in) that: (a) exploit steady state relationships and long-run historical averages, (b) match some second-order moments of the time series (e.g., by the simulated method of moments, SMM), or (c) estimate in the data reduced-form or partial equilibrium relationships (a subset of relationships) that must be satisfied by the model. Micro evidence is also frequently cited as the main data source used to pin down a number of those structural parameters, as it diminishes the danger that the calibration may become an exercise to fit the macro data.

Given our model specification, there are 6 structural (non-policy) parameters and 2 policy parameters that affect the dynamics of the NOEM model. The structural (non-policy) parameters include the intertemporal discount factor, $\beta$, the inverse of the intertemporal elasticity of substitution, $\gamma$, the inverse of the Frisch elasticity of labor supply, $\varphi$, the elasticity of substitution between Home and Foreign bundles, $\sigma$, the share of locally-produced goods, $\xi$, and the Calvo price stickiness parameter, $\alpha$. The policy parameters include the sensitivity of the monetary policy rule to deviations from the inflation target, $\psi_{\pi}$, and from the potential output target, $\psi_{x}$.

In the dynamics of the flexible price model, there are just 4 structural (non-policy) parameters and 1 policy parameter. The intertemporal discount factor, $\beta$, and the Calvo parameter, $\alpha$, are not present, as there is no Phillips curve relationship under monetary neutrality. By definition, current output and potential output are the same object in the flexible price economy, so there is no need for monetary policy to respond to the output gap as there will be no gap. Hence, the policy parameter $\psi_{x}$ becomes irrelevant for the resulting endogenous dynamics.

The calibration of these (policy and non-policy) structural parameters can be done many different ways, leading to a wide range of possible values. We illustrate some of the most conventional calibration strategies and the dispersion of their suggested parameter values in the context of parameterizing our model (our DGP process):

I. Exploit steady state relationships. ${ }^{21}$ Some parameters affect both the steady state as well as the dynamics of the model, so we can pin them down using the steady state relationships and long-run macroeconomic time series averages. Steady state relationships and data on long-run average import shares and interest rates allow us to identify the intertemporal discount factor, $\beta$, and the share of locally-produced goods, $\xi$. As noted before, the parameter $\beta$ enters into the Phillips curve specification and together with the Calvo parameter, $\alpha$, determines its slope. The slope of the Phillips curve is critical to the trade-off between domestic inflation and global slack in our model and, therefore, the impact of monetary non-neutrality. Hence, exploiting the steady state relationship linking $\beta$ to the long-run real interest rates facilitates our task. However, the Calvo parameter $\alpha$ does not enter into the deterministic steady state and other alternative sources of information are needed to identify it and - ultimately — to validate the 'global slack hypothesis' articulated in our model under the assumption of price stickiness. ${ }^{22}$

unless the data-generating process has changed significantly over time. If that is the case, the pre-sample calibration may not be of much use to avoid the circularity anyway or may bias the results by not recognizing a possible structural change.

${ }^{21}$ Our model does not explicitly incorporate trend growth (whether endogenous or exogenous), but often the data appears to be trending upwards. Ferroni (2011) offers an interesting analysis of the merits of pre-filtering the data before estimating a DSGE model vs. estimating the trend and deviations around trend simultaneously. Either way, it should be noted that balanced growth path considerations may impose additional restrictions that we can use to calibrate certain parameters. See, e.g., the discussion in Chari et al. (2002) on the parameterization of preferences.

${ }^{22}$ As carefully documented in Ríos-Rull et al. (2011), the specification of the building blocks of the model can be critical 
II. Exploit available micro-level data or second-order moments of the macro-level data. ${ }^{23}$ Often the micro- and macro-level data provide different indications for calibrating a parameter, and naturally different calibrations can lead to significantly different model predictions. Reconciling the micro and macro evidence is often a challenge for researchers, as it may be a signal that a model is misspecified. The proponents of Bayesian estimation would argue that an advantage of this technique is that this wide range of often contradictory evidence on key structural parameters can always be subsumed into priors so that the researcher does not have to take a stand one way or another. From this view, Bayesian techniques let the data reveal the parametric values that are most favored by the macro time series data. However, while micro evidence can be incorporated in the formation of priors intended to explain macro aggregates, micro data do not enter into the likelihood function which conventionally incorporates macro data alone. Moreover, if model misspecification is indeed an issue, the Bayesian estimates may fall far from their true values anyway because the misspecified data-genetaring (DGP) process introduces a bias in attempting to better fit the model to the data. Working with simulated data, however, we control when misspecification occurs and can concentrate on assessing the ability of the Bayesian methods to accurately identify the parameters of the model when the prior information is as wide for some of these structural parameters as the current empirical evidence would suggest.

The moments used in calibration should be as informative as possible, in the sense of being distinctly linked to the structural parameters. While it is often difficult to evaluate in a stringent way whether that is the case, the selection of moments may be guided by economic theory. Indeed, it is recommendable to consider aspects of the data that are important because they shed light on the merits of the theory (whether it can be refuted) or because they matter most for policy analysis. In turn, one can also consider other objects such as impulse response functions (estimated from VARs) as the most relevant feature of the data to match. This is particularly common when characterizing the transmission of monetary shocks in the model and comparing that to the data. While that plays a role in how we think about calibrating the model, we'll discuss it later more in the context of Bayesian estimation - as in our exercise we are able to determine how well the estimation does in recovering the true impulse response functions (impulse response identification), not just how well it does in recovering the true structural parameters (parameter identification).

Frisch elasticity of labor supply: The Frisch elasticity of labor supply, $\frac{1}{\varphi}$, is commonly identified in the literature on the basis of micro-level data. Pencavel (1986) reports that the typical point estimate of the labor supply elasticity for men is 0.2 among the studies he surveyed, with a range of estimates going from 0 to 0.45. Other classical surveys on the empirical micro literature include Card (1994) and Browning et al. (1999), and more recently the one by Keane (2010). Most micro studies consistently indicate that the Frisch elasticity of labor supply lies below 1. Based on that micro evidence, we set the Frisch elasticity of labor supply $\frac{1}{\varphi}$ at 0.2 to match the representative point estimate reported by Pencavel (1986). ${ }^{24}$

to the steady state relationships that can be used to estimate some of the key propagation parameters of the model. They illustrate that point with examples of alternative preference specifications that constrain the Frisch elasticity of labor supply $\varphi$ in steady state and others that do not. However, altering preferences or other key building blocks of the model may also impose other unwanted features that are inconsistent with the data in other dimensions (e.g., changing the trade-off between income and substitution effects on labor, etc.). In any case, in our model we cannot parameterize $\varphi$ based on the steady state relationships reported in Table 4.

${ }^{23} \mathrm{By}$ second- or higher-order moments, we often refer to the covariance structure of the macro data or the autocorrelation of the time series data.

${ }^{24}$ Ríos-Rull et al. (2011), however, emphasize the heterogeneity in the micro data measurements used to parameterize $\varphi$. For instance, the survey of Pencavel (1986) refers exclusively to the labor supply of men and does not distinguish between the intensive and extensive margins on employment. Depending on the question at hand, the evidence provided by Pencavel 
Macro-estimates can also be used in order to pin down the Fisch elasticity of labor supply. Rotemberg and Woodford (1998b) and Rotemberg and Woodford (1998a) argue that the inverse of the Frisch elasticity needs to be as low as $\frac{1}{9.5}=0.10526$ to match the relatively weak observed-response of real wages to monetary disturbances and other macro features of the labor market. Ríos-Rull et al. (2011), using a standard Neoclassical growth model investigate conditions under which the impulse response functions of wages can be used to identify the labor supply elasticity. They conclude that to match the response to a neutral technology shock requires a low value of the inverse of the Frisch elasticity of labor supply, while matching the response to invesment-specific technology shocks and preference shocks requires a high value of the same parameter. Reconciling those different macro estimates is always contentious, as it presumes that we can properly identify the shocks themselves and their contribution to the business cycle (as the calibration of the labor supply would depend on the relative importance of each shock over the cycle).

Intertemporal elasticity of substitution: The elasticity of intertemporal substitution, $\frac{1}{\gamma}$, is often identified on the basis of macro-level data. The empirical literature provides a wide range of possible values for this parameter. Even when using essentially the same macro data on aggregate consumption and real yields, researchers seem to arrive at different conclusions. On one hand, Hall (1988) uses the conditional consumption Euler equation to estimate the elasticity of intertemporal substitution from the co-movement of aggregate consumption with the real yields. Hall (1988) argues that the inverse of the elasticity of intertemporal substitution $\gamma$ is likely above 5 based on the observed data. Yogo (2004) finds general agreement with Hall (1988)'s findings on a sample of eleven developed countries, while also taking account of the problem of weak instruments in the estimation. On the other hand, most business cycle models -including many international business cycle models - typically assume a much lower value for the inverse elasticity $\gamma$. Lucas (1990), for example, using the same macro data argues that: "If two countries have consumption growth rates differing by one percentage point, their interest rates must differ by $\gamma$ percentage points (assuming similar time discount rates). A value of $\gamma$ as high as 4 would thus produce cross-country interest differentials much higher than anything we observe, and from this viewpoint even $\gamma=2$ seems high." ${ }^{25}$

The inverse of the intertemporal elasticity of substitution, $\gamma$, can also be calibrated with the simulated method of moments (SMM), as in Chari et al. (2002). ${ }^{26}$ Following their example, we can use macro data on international relative prices or trade to pin down this parameter. In fact, we set the preference parameter $\gamma$ at 5, as in Chari et al. (2002) and Martínez-García and Søndergaard (2008), in order to match the volatility of the real exchange rate relative to output. Given our specification of preferences and the assumption of complete international asset markets, the standard deviation of the real exchange rate ought to be proportional to the standard deviation of relative consumption, and the constant of proportionality is

(1986) might be out-dated or might simply be too narrow missing important dimensions of the labor market. This suggests that scholars may want to look at other sources of information to calibrate this parameter depending on the question addressed in their work.

${ }^{25}$ For consistency, we have replaced here the notation in Lucas (1990) for the inverse of the elasticity of intertemporal substitution with ours (i.e. with $\gamma$ ).

${ }^{26}$ The simulated method of moments (SMM) can be applied simultaneously to calibrate other structural parameters such as the inverse Frisch elasticity of labor supply $\varphi$. We could match the volatility of employment (or hours worked) in the data to pin down the labor supply elasticity. This may not be trivial anyway, because the labor supply equation embedded in our framework is known to be very restrictive (and possibly misspecified). As it will be discuss later, the elasticity of substitution between Home and Foreign bundles, $\sigma$, can also be calibrated in this fashion. It is recommended that the moments and the data used to calibrate these parameters under the SMM approach do not correspond with the same moments and data that we aim to explain, so that circularity does not become an issue. By focusing on labor or, most significantly, on trade data, we also provide some cross-validation for the theory (on the real effects and international transmission of monetary shocks) outside its core. 
given by the parameter $\gamma$. As we vary $\gamma$, hence, we are changing the volatility of the real exchange rate in relation to the volatility of relative consumption as well. ${ }^{27}$

In a bond economy, a similar exercise can be conducted with similar results for the calibration of this parameter. The equilibrium condition that forms the basis of these calculations is often known as the international risk-sharing condition, and it relates the expected change in the real exchange rate to the expected consumption growth differential between the two countries (based on all available past and current information). Under complete asset markets it holds for every possible state of nature. Together with the uncovered interest rate parity condition that relates expected changes in the real exchange rate to real interest rate differentials across countries, it provides the theoretical underpinnings for Lucas (1990)'s argument. However, Lucas (1990) points to data on consumption and yields rather than real exchange rates to reach a very different conclusion on the proper calibration of the inverse intertemporal elasticity of substitution $\gamma$ than Chari et al. (2002). This case illustrates how the choice of the empirical moments and the data to match is not inconsecuential when following the SMM approach.

Intratemporal elasticity of substitution between Home and Foreign goods: The elasticity of intratemporal substitution between Home and Foreign goods, $\sigma$, is commonly identified on the basis of macro-level data. Based on empirical estimates of trade models, it is generally noted that plausible values of the U.S. elasticity of intratemporal substitution lie between 1 and 2. However, it is also known that the model predictions are quite sensitive to the choice of $\sigma$. For instance, we know that in the model investigated in this paper the parameter $\sigma$ affects the magnitude and the inequality $\sigma \gtreqless \frac{1}{\gamma}$ determines the sign of the propagation of foreign shocks into the domestic economy. Here we follow in the footsteps of Backus et al. (1994) and Chari et al. (2002) setting the elasticity $\sigma$ to be equal to 1.5. We could also use macro data on trade and international relative prices to pin down this parameter following the logic of the SMM method. As implied by the relationships in Table 2, the trade balance must be proportional to terms of trade in the model and their correlation must be equal to one always. However, as we vary the parameter $\sigma$, we change the ratio between the volatility of the trade balance and the volatility of terms of trade (or the real exchange rate) approximating its empirical counterpart. ${ }^{28}$ That gives us a way to relate the calibration of this parameter to macro data on trade as well.

Frequency of price adjustments: The Calvo parameter, $\alpha$, is often identified using micro-level data. However, the empirical micro literature provides a wide range of possible values for $\alpha$. The convention prevalent in most of the NOEM literature is that prices remain unchanged for on average four quarters (implying that $\alpha=0.75$ ), as noted by Chari et al. (2002) among others. Bils and Klenow (2004) shook this perception with evidence on U.S. CPI data suggesting that the median frequency of price changes implied a duration of merely 4.3 months. Klenow and Kryvtsov (2008) and Nakamura and Steinsson (2008) report a similar range of $7-10$ months based also on U.S. CPI data, while prices in the Euro-Area CPI appear to change even less frequently according to Dhyne et al. (2006). The evidence surveyed by Taylor (1999) and

\footnotetext{
${ }^{27}$ The model used by Chari et al. (2002) and Martínez-García and Søndergaard (2008) allows for deviations of the law of one price to explain fluctuations in the real exchange rate, under the assumption of local-currency pricing. Our model, in turn, under producer-currency pricing only permits deviations from purchasing power parity to arise due to differences in the consumption basket across countries. That, in turns, as can be seen in Table 2 implies that real exchange rates are proportional to terms of trade by a factor of less than one. Therefore, terms of trade are always more volatile than the real exchange rate in our model, which is counterfactual. An alternative would have been to match the volatility of terms of trade relative to output instead, which is likely to give us a lower value for $\gamma$. Instead, for expositional purposes and simplicity, we simply adopt the parametric value of 5 used by Chari et al. (2002) and Martínez-García and Søndergaard (2008).

${ }^{28}$ For a similar strategy, see Heathcote and Perri (2002).
} 
more recently the micro price studies reviewed by Klenow and Malin (2010) are consistent with the view that prices change on average closer to once a year (after excluding most short-lived price changes). We adopt the standard value of 0.75 for the degree of price stickiness $\alpha$, implying an expected price duration of four quarters, to be consistent with the NOEM literature.

Policy parameters: We assume that the Taylor rule is inertial of the extrinsic type, as the original rule proposed in Taylor (1993) did not include intrinsic inertia. The parameters of the Taylor rule are often estimated to fit the data, but quite often the values estimated for $\psi_{\pi}$ and $\psi_{x}$ are close to those proposed by Taylor (1993) on the basis of his exploration of the behavior of the U.S. Fed Funds rate during the period 1987 - 1992. We adopt the policy parameters estimated for the U.S. by Rudebusch (2006) jointly with the $A R(1)$ stochastic process parameters for the monetary policy shocks, and assume they are identical in both countries. Hence, we set $\psi_{\pi}$ at 1.24 and $\psi_{x}$ at 0.33 which are fairly close to the values of 1.5 and 0.5 respectively proposed by Taylor (1993).

Parameters related to the exogenous shock processes. Typically, the identification of the parameters associated with the exogenous shock processes often involves two steps. The first step consists in derivingwhenever possible - a time series for the realization of the shocks. To do so we have to map the data as closely as possible to the model variables by using consistent measures for the macro aggregates. The use of a few key equilibrium conditions and some previously calibrated structural parameters is often necessary. However, it is preferable if the parameters used to infer the realization of the shocks are calibrated on the basis of long-run historical data averages and steady state relationships - particularly to avoid parametric values that have been selected with the same time series macro data that we would need to derive the realization of the shocks. Moreover, it is also preferred if deriving a realization of the shocks does not require us to use the calibration of some of the key propagation parameters that are central to our question. The second step involves characterizing the dynamics of the shock processes, generally by estimating a pre-specified stochastic process on the time series realization derived in the first step.

In our model, there are just two country-specific shocks that we have to consider: productivity shocks and monetary shocks. The two key equations needed to infer a realization of these shocks are the production function and the monetary policy rule. Under the assumption of linear-in-labor technologies, we can map the labor productivity of these countries into the productivity shocks of the model denoted as $\widehat{a}_{t}$ and $\widehat{a}_{t}^{*}$. In this case, no structural parameters are needed to infer a realization of the shocks. ${ }^{29}$

For the parameterization of the VAR(1) productivity shock process, we follow Heathcote and Perri (2002) which use real GDP and employment data from 1973 to 1998 to identify a realization of the productivity shock for the U.S. and a foreign aggregate that bundles together 15 European countries, Canada and Japan. Their approach is similar to the one we would need for our model (although they adjust the labor data with a labor share set at 0.64 as their model includes capital). Based on their estimates, we set $\delta_{a}$ (the persistence parameter) at 0.97 and $\delta_{a, a^{*}}$ (the cross-country spill-over parameters) at 0.025 . The volatility $\sigma_{a}$ is set at 0.73 and the correlation between domestic and foreign innovations $\rho_{a, a^{*}}$ at 0.29 . Monetary and productivity innovations are assumed to be uncorrelated with each other, and we also rule out by construction the presence of spillovers between monetary and productivity shocks.

\footnotetext{
${ }^{29}$ In a richer model with capital and labor as inputs in a Cobb-Douglas production function and with competitive factor markets, the capital share in the production function can be determined through the steady state relationships (see, e.g., the discussion in Ríos-Rull et al. (2011)). With the capital labor share as well as data on the stock of capital, hours worked and aggregate output, we can infer a time series for the so-called Solow residual.
} 
The parameterization of the monetary policy shocks is a bit less straightforward. We can still derive a realization of the monetary policy shock from the monetary policy rules specified for each country (which are assumed to be inertial of the extrinsic type), but that requires taking a stand on the choice of the policy parameters $\psi_{\pi}$ and $\psi_{x}$ which are known to be very relevant to assessing the real effects of monetary policy. In other words, the policy parameters are central to the propagation mechanism and policy trade-offs that we are aiming to investigate, but cannot be pinned down through a convenient set of steady state relationships. One conventional approach is to jointly estimate the Taylor rule parameters and the specification of the monetary shock process - hence identifying the monetary shocks with the residual of that regression. The danger of the approach is that the misspecification of one of its parts (the shock process or the reaction function for monetary policy) may bias both the estimates of the monetary shock process as well as the estimates of the policy parameters.

For simplicity, we adopt the policy parameter values estimated for the U.S. by Rudebusch (2002), i.e., $\psi_{\pi}=1.24$ and $\psi_{x}=0.33$, for both countries. For the VAR(1) monetary shock process, we follow the estimates of Rudebusch (2002) as well in setting $\delta_{m}$ at 0.92 and $\sigma_{m}$ at 0.36 for the persistence and volatility in both countries. We complete the description of the dynamics by choosing the correlation between Home and Foreign monetary innovations $\rho_{m, m^{*}}$ to be 0.5 as in Chari et al. (2002).

Data simulation from the model. We use standard perturbation methods to solve the model. We derive a deterministic, zero-inflation steady state. We log-linearize the equilibrium conditions around the steady state. This local approximation is accurate for arbitrarily small exogenous shocks that are bounded within a neighborhood of the steady state. Then, we solve the resulting linear rational expectations model based on the generalized Schur decomposition method (see, e.g., Villemot (2011)) and estimate it using a Monte-Carlo based optimization routine, as implemented by the software package Dynare. ${ }^{30}$ We use this plain-vanilla log-linear approximation of the workhorse NOEM model as our Data-Generating Process (DGP). Estimating this log-linearized model on the basis of simulated data (rather than actual data) allows us to clearly distinguish between model misspecification biases and identification issues in-sample, as well as to highlight the problems associated with identification of model parameters using conventional Bayesian techniques.

We simulate the full model over 11,000 periods, and drop the first 1,000 observations of each series to exclude any effect of the initial conditions on the simulation. Based on this single realization, we keep a long sample with the remaining 10,000 periods of the simulation but also select three shorter sub-samples of 160 observations each. We use Bayesian estimation methods on the long and short sub-samples to investigate the ability of these techniques to recover the true parameters of the model. The short sub-samples correspond to 40 years of quarterly data and are meant to capture a fairly long, but plausible length for time series international macro data. The long sample corresponds to 2,500 years of quarterly data. While unrealistically long, this 10,000-period long sample allows us to illustrate the estimation gains that can be attained as the availability of time series data for estimation becomes arbitrarily large (relative to more conventional sample

\footnotetext{
${ }^{30}$ The proliferation of software to perform Bayesian estimation (e.g., the Dynare software) has significantly reduced the cost of implementing Bayesian econometrics. We apply Dynare in our paper in part to illustrate the easiness of performing a Bayesian estimation exercise, as this software is free and widely used. The downside of this has been that estimation (Bayesian estimation) has become common practice, but less effort is made to explain or understand why and for what purpose. One of the main themes of this paper is to raise awareness of the importance of understanding the limitations of the method as well as to encourage researchers to consider the circumstances and questions for which it might be helpful in their work.
} 
sizes for macro aggregates).

Figure 2.A shows the long sample simulation corresponding to Home and Foreign output. It also marks the three 160-period short samples that we have selected for our Bayesian exploration from those two series. Figure 2.B plots the long and short samples corresponding to Home and Foreign (quarter-on-quarter) inflation, and Figure 2.C similarly depicts the long and short samples corresponding to terms of trade and the trade balance. Figure 3 further describes the simulated data that we will use in our Bayesian estimation exercises by plotting Home and Foreign output, Home and Foreign inflation, terms of trade and the trade balance for each one of the three short samples side-by-side. Figure 4 completes the description of the data by showing scatter plots for domestic inflation and domestic output, for domestic inflation and foreign output and for domestic inflation and terms of trade for each one of the sub-samples. The univariate evidence provided in Figure 4 indicates that the within-sample linear correlation between domestic inflation and the other endogenous variables can be very different across the three sub-samples that we consider here. As all these figures illustrate, the time path of each of these endogenous variables can be quite different depending on the sample that we select.

Whenever it is pertinent to simulate data from another version of the model such as the flexible price specification (the RBC model), we maintain invariant the realization of the exogenous shocks and the strategy to select a long sample and three shorter sub-samples while varying exclusively the structure of the model and its endogenous propagation.

\section{[Insert Figures 2.A,2.B,2.C,3,4 about here.]}

We want to contrast the estimation results of these three short samples of 160 observations each against a longer sample of 10,000 observations to investigate the practical importance of the well-known 'peso problem' in small-sample inference. As noted by Evans (1996) in his survey of economic anomalies associated to the 'peso problem', this issue arises when the realized or ex post frequencies of the observables within sample differ significantly from their ex ante distribution - implied by the true DGP process - leading to distorted econometric inferences. The 'peso problem' can be confounded in the data with the misspecification of the DGP process, but that is something we can rule out by construction when working with simulated data. In our exercise, the population distribution is potentially known to the econometrician as the true DGP process is taken as given. However, the population distribution is a function of the model parameters that need to be recovered and identified.

When the 'peso problem' becomes a major concern is when the sample moments computed with the available macro time series do not coincide with the population moments that would have been used by economic agents when forming their expectations and making their economic decisions. In the context of the model postulated here, those population moments and the distribution are assumed to be known by the agents but not to the econometrician who only knows the distribution as a function of a number of unobserved parameters. Hence, the empirical problem that comes from small samples exists precisely because the data is insufficient to identify the population distribution and map them into the corresponding model parameters. That is not only a problem for the evaluation of the model (or for model comparison), but it can also seriously distort the policy analysis and forecasting for which these estimated models are often used even though the true DGP process is actually known without any uncertainty.

In Table 7, we summarize how the features of these time series data (the long and short samples) compare against the theoretical moments implied by the NOEM model. It is interesting to note how the divergence 
between the sample moments and the theoretical moments can be very large for samples of 40 years of quarterly data (160 observations) and even be significant for a sample of 10,000 observations, especially for Home and Foreign output. In spite of the inherent symmetry of the model, the sample moments can differ a lot between the Home and Foreign country in some cases. The mean and the volatility of the series seem to be quite off from their population counterparts, but even persistence at long lags can differ. Looking at the skewness and kurtosis of the long- and short-sample series using the Jarque-Bera statistic also gives us a different reading about the normality of the observed series, although it is already known that the Jarque-Bera statistic is less reliable in small samples. Indeed, all this evidence suggests that in the context of our simulated data a conventional sample size of no more than 160 observations can be prone to the 'peso problem'.

[Insert Table 7 about here.]

\section{Bayesian Estimation}

The previous section gives us a broad perspective on the conventional calibration approach. The goal, however, was two-fold: on the one hand, it helps us motivate the standard calibration chosen for the workhorse NOEM model that we use as our benchmark data-generating process (DGP); on the other hand, it serves also to introduce a number of different (sometimes contradictory) sources and evidence that will make their way into our selection of priors for Bayesian estimation. The data that we use in all our estimations is obtained from simulating the NOEM model under the benchmark calibration summarized in Table 6. We assess the strength of the identification strategy implemented through Bayesian estimation techniques in this controlled environment where we can determine in each of our experiments how much information does the econometrician have available and whether the model structure to be estimated is misspecified to some degree or not.

In the next two subsections, first, we give a brief overview of the assumptions we make about priors for the structural parameters of the model and we explain how the available information factors into our choices; and, second, we design a number of experiments intended to illustrate how the strength of the identification may depend on features that are out of the researchers control (for instance, the prevailing monetary policy regime or the sample size) or may result from the choices the researcher makes in attempting to bring the model closer to the data (for example, in selecting the number of observables or even the number of non-structural shocks). In all of our experiments, we emphasize also the importance of the sample size in identification - suggesting that 40 years worth of quarterly data which is considered a long time series in international macro, may not be "long enough" to get precise identification through Bayesian estimation.

\subsection{Eliciting Priors}

All our priors are summarized in Table 8 and Figure 5, exluding the three parameters that we normalized before and take as given here. We only consider prior densities of the beta, gamma, inverse gamma, normal, and uniform distributions as well as the degenerate distribution that puts mass one on a single value. We choose among these priors because they are overwhelimingly the distributions most used in the Bayesian estimation literature. In a few cases, we rely on transformations of the parameters of interest in order to 
ensure the range of values that the parameter can take according to theory (indicated in Table 6) conforms with the range of values that are supported by the preferred prior distribution function. In cases where a transformation is pertinent, a linear transformation always suffices to help us match the ranges of the prior distribution with those implied by theory. We also impose on all cases that the prior mean must be equal to the true value of the parameter in our calibration to be consistent with our own views about the data used to parameterize the model. In turn, we choose the prior distribution as well as the dispersion to reflect the degree of uncertainty that exists regarding those parameter values.

Since we match the prior mean to the true parameter used to calibrate and simulate the model, the main point of the estimation is to see whether the Bayesian techniques applied to observable macro time series can contribute to reduce in some sense the dispersion (or uncertainty) surrounding the true value of these parameters. The dispersion arises from the evidence about the parameters provided by the alternative sources that we have discussed before. In other words, can we expect Bayesian techniques to help us reveal additional information that is not already incorporated in the priors? If so, how large does the sample size have to be for identification (and how significant can the introduction of small-sample biases be)?

We maintain these prior distributions invariant in all our subsequent estimations experiments, taking them as given. While we acknowledge that there is no unique way of eliciting priors and that other researchers may reach different conclusions on the selection of priors by emphasizing other sources of information not covered here or weighting differently the ones we have mentioned before, we want to take the role of priors seriously as reflecting our ex ante views and beliefs about these parameters. Moreover, by keeping the priors invariant, we make comparisons across models and the implementation strategies for estimation much more straightforward.

\section{[Insert Table 8 and Figure 5 about here.]}

Structural Parameters. As it is conventionally done, we use a degenerate prior for the intertemporal discount factor $\beta$ and fix it at 0.99 . We impose a degenerate prior on $\beta$ that targets an average yearly interest rate of $4 \%$ as in our calibration, so this should not cause a bias in our estimates of the other parameters. This value is also based on the long-run historical average of the real interest rate (nominal rate minus realized inflation) that does not enter into the set of observables that we use to estimate the model.

While we do not use a degenerate prior for the share of locally-produced goods in the consumption basket $\xi$, we choose a tight prior to recognize that this parameter is tied to the import share through the steady state and hence cannot deviate too much from the import share's historical average. We use a Beta distribution for the prior and transform the parameter to $2 \xi-1$ to ensure that the range of possible values of this transformed parameter corresponds with the domain of the Beta distribution. Accordingly, we center the prior of the transformed parameter around 0.88, which implies a prior mean for $\xi$ that is equal to the calibrated value of 0.94 . We also impose a small standard deviation of 0.01 , which implies that the prior Beta is single-peaked and puts most of its mass within a small neighborhood around the mean. This prior specification emphasizes the conventional view that with the historically low import share observed for the U.S., one should not expect ex ante the parameter $(1-\xi)$ in the model - which defines the degree of trade openness - to be too large.

Other critical model parameters for monetary non-neutrality and the strenght of the propagation mechanism in the NOEM model are the remaining (non-policy) structural parameters: the inverse of the intertem- 
poral elasticity of substitution, $\gamma$, the inverse of the Frisch elasticity of labor supply, $\varphi$, the elasticity of substitution between Home and Foreign bundles, $\sigma$, and the Calvo price stickiness parameter, $\alpha$. As we discussed earlier, the different information sources that can be brought to bear in calibrating these parameters are sometimes hard to reconcile with each other or simply give us a wide range (and very different perspectives) of possible values for these parameters. As Bayesian proponents would argue, all those concerns can be effectively subsumed into a wide-enough prior distribution so that ultimately we let the data pick the most likely candidate for us.

In that spirit, we adopt the Gamma distribution centered around 5 for $\gamma$ and $\varphi$, ensuring that the prior means correspond to their true values according to our benchmark parameterization of the data-generating process (DGP). In turn, we impose a wide standard deviation of 5 for both $\gamma$ and $\varphi$ to encompass the wide range of values considered as plausible in the calibration literature for both paramters. This standard deviation also implies a strictly decreasing prior distribution that puts higher mass on values below the mean than it does on values above it. We adopt the Gamma distribution centered around 1.5 for $\sigma$, making the prior mean correspond to the true value of the parameter in the benchmark calibration. We impose a wide standard deviation of 1 to recognize the importance of this parameter for the international transmission of shocks (especially depending on whether the parameter is greater or smaller than $\frac{1}{\gamma}$ ) and to capture the uncertainty surrounding its value. This prior specification results in a wide range of plausible values for the intratemporal elasticity of substitution between Home and Foreign bundles, with a unimodal distribution skewed towards the left.

We adopt the Beta distribution centered around 0.75 for the Calvo parameter, $\alpha$, also ensuring that the prior mean corresponds to its true value in our benchmark calibration. ${ }^{31}$ For $\alpha$, we pick a wide prior Beta distribution with standard deviation 0.07. The Calvo parameter $\alpha$ indicates the fraction of firms that are not able to re-optimize in any given period, so we favor a unimodal Beta prior that internalizes the empirical evidence referenced earlier - mostly micro evidence - suggesting 0.75 could be on the high end of the plausible values for this parameter. This prior distribution puts little mass on values of the parameter range above 0.9 (which imply expected durations of more than ten quarters) and below 0.5 (which imply expected durations of less than two quarters).

Similarly, other key model parameters for monetary non-neutrality and the strength of the propagation mechanism in the NOEM model are the policy parameters $\psi_{\pi}$ and $\psi_{x}$. We center the policy parameters around their calibrated values, but we impose an Inverse Gamma distribution for both of them and select fairly wide priors. The parameter for the policy response to deviations from the inflation target needs to be transformed in order to be consistent with the domain of the Inverse Gamma distribution and to rule out violations of the Taylor principle where the Blanchard-Kahn conditions are not satisfied because monetary policy is not aggressive enough. Hence, we estimate $\psi_{\pi}-1$ with a prior centered at 0.24 that implies a prior mean of 1.24 for the corresponding policy parameter. The prior mean of the sensitivity to deviations from potential output $\psi_{x}$ is maintained at 0.33 , which also coincides with the benchmark calibration. Monetary policy parameters are somewhat less controversial in the literature, so we select a prior standard deviation

\footnotetext{
${ }^{31}$ The Calvo parameter $\alpha$ is of great importance for it explains monetary non-neutrality in the short-run dynamics of the model. However, $\beta$ and $\alpha$ tend to be hard to identify simultaneously through the Phillips curve relationship. The Calvo parameter $\alpha$ is regarded as more uncertain than the parameter $\beta$, and of greater interest to the researcher because it is directly connected to the nominal friction of the model (the degree of nominal rigidity). Hence, we follow the costumary practice in the Bayesian estimation literature of imposing a degenerate prior on $\beta$ which is meant (at least in part) to facilitate a more precise estimation of the Calvo parameter $\alpha$.
} 
of 2 for both of them that is not too wide.

Parameters of the Shock Processes. Priors for certain features of the exogenous shock processes may have to be rather diffuse, even though we have realizations of the Solow residuals and the monetary residuals that we can use for guidance. The selection of priors on parameters related to the shock processes conventionally involves imposing restrictions on the feasible range of some of these parameters to discard draws from regions of the parameter space that may lead to non-stationary solutions or that are simply deemed unrealistic to match the data. For starters, the prior distributions for the partial autocorrelations of both shocks is restricted to lie within the interval $(0,1)$, so as to rule out negative values for $\delta_{a}$ and $\delta_{m}$ which seem unsopported by the data. However, that does not suffice to also ensure the stationarity of the productivity $V A R(1)$ process as we have to consider the possible values of the spill-over parameter $\delta_{a, a^{*}}$.

For simplicity, we adopt a degenerate distribution for the persistence of the productivity shock $\delta_{a}$ to match its calibrated value of 0.97 , as there seems to be broad agreement that the Solow residuals are pretty persistent. Adopting a degenerate prior for $\delta_{a}$, in turn, allows us more flexibility to impose a diffuse prior on the key spillover parameter $\delta_{a, a^{*}}$ and still guarantee the stationarity of the stochastic process for productivity. It follows from the choice of $\delta_{a}$ that in order for the $\operatorname{VAR}(1)$ process that describes the productivity shocks to remain stationary (with both its eigenvalues inside the unit circle) we would need $\delta_{a, a^{*}}$ to be between -0.03 and 0.03. We transform the spill-over parameter to become $\frac{1}{2}+\frac{1}{2} \frac{\delta_{a, a^{*}}}{0.03}$ so that its range can be defined over the $(0,1)$-interval and select the Beta distribution as our prior. We center the distribution around 0.91667 to be consistent with our calibration of $\delta_{a, a^{*}}$ to be equal to 0.025 . Moreover, we set the prior standard deviation at 0.05 . This Beta prior is unimodal and skewed to the right to explicitly incorporate the common view that positive spillovers in productivity are more plausible than negative ones.

Having imposed extrinsic inertia on monetary policy, the first-order autocorrelation of the monetary shocks $\delta_{m}$ ought to be positive and high in order to match the parsimonious interest rate movements that we observe in the data. We reflect this in our prior for $\delta_{m}$ by restricting the parameter space to the interval $(0,1)$ and by selecting a Beta distribution centered around its calibrated value of 0.92 with a prior standard deviation equal to 0.02. This implies that the prior Beta for $\delta_{m}$ is unimodal, and it recognizes that the empirical evidence seems to favor values consistent with high persistence of the monetary shock. The prior means of the productivity shock and monetary shock volatilities, $\sigma_{a}$ and $\sigma_{m}$, are set at their calibrated values of 0.73 and 0.36 , respectively. As customary, we select an Inverse Gamma distribution to represent the prior distribution of both volatility parameters. However, we impose a large standard deviation of 5 on both cases leaving it open for the data to determine the contribution of each shock to explain the endogenous volatility.

Finally, we restrict the range of the parameter space for the cross-country correlation of innovations $\rho_{a, a^{*}}$ and $\rho_{m, m^{*}}$ to lie in the $(0,1)$-interval. We select the Beta distribution again because it gives us more flexibility to match the calibrated parameters with the prior mean than the uniform distribution while imposing a relatively uninformative prior. We choose rather diffuse priors for these cross-country correlations because these parameters can be crucial for the dynamics of the model (e.g., for the cross-country endogenous correlations), but their values are often greatly debated in calibrated and estimated models. We center $\rho_{a, a^{*}}$ at 0.29 with a standard deviation of 0.18 , and $\rho_{m, m^{*}}$ at 0.5 with a standard deviation of 0.22 . As a result, the prior Beta distribution for the cross-correlation of the productivity innovations is skewed toward the left reflecting the fact that very large correlations are not what is commonly reported in the empirical literature or consistent with the reported estimates on the Solow residuals. In turn, our prior Beta for the 
cross-correlations of the monetary innovations is somewhat similar to imposing a non-informative uniform distribution, except that it puts significantly less mass on the extremes of the parameter space. In choosing this specification, we are indicating that our understanding of the proper value for this correlation is rather limited.

In some of our estimation exercises, we also incorporate a measurement error term modelled in terms of pure white noise. This is a non-structural shock added to the especification of the estimated model for the purposes of increasing the set of observables variables that we consider in the estimation. The shock process is characterized with only one parameter, the volatility term $\sigma_{v}$. The prior mean of this ad hoc measurement error shock volatility, $\sigma_{v}$, is naturally set at a low value of 0.10 (as this shock is considered to have only a "small" or "marginal" contribution to business cycle). As customary for all volatility parameters, we also select an Inverse Gamma distribution to represent the shape of the prior distribution. We impose a standard deviation of 1 allowing for the possibility that this volatility may take larger values than what we would expect, but not too large.

\subsection{Estimation}

The model is estimated based on three samples of 160 observations each generated from our benchmark calibrated NOEM model, and a long sample of 10,000 observations. A sample of 160 observations is equivalent to 40 years of quarterly macro data, which is typically a longer time span than most estimated open-economy macro models can cover due to data limitations and other coverage problems, due to the implicit assumption of an invariant monetary policy regime over the entire period, etc. The long sample of 10,000 periods is used as a reference to assess the precision of the estimation in small samples and evaluate the severity of the so-called 'peso problem', as mentioned earlier. In all of our experiments we take the data-generating process (DGP) as given and rule out the possibility of model misspecification. We also keep the priors invariant. In turn, we investigate the sensitivity of Bayesian inference in small-samples to the implementation strategy that an econometrician dealing with this model will have to develop in three dimensions: (a) the decision of whether to prefilter the data or not prior to estimating the model, (b) the choice of observable variables for the estimation, and (c) the choice of whether to include non-structural shocks in the estimation.

In this section, we shall revise all three aspects of the implementation in the context of applying the Bayesian techniques to estimate the NOEM model and their effect on structural identification in small samples.

Pre-filtering the Data. The NOEM model posits monetary non-neutrality and opens the door to the international propagation of shocks. Given our emphasis on monetary shocks and monetary non-neutrality, it would seem pertinent to include both real and nominal variables in the set of observables that we estimate in order to help us detect those real effects. In order to avoid stochastic singularity in Bayesian estimation, we must have the same number of observable variables as structural shocks in our model. Since we have monetary and productivity shocks that are country-specific, that means we have four structural shocks and we accordingly we should have four observable variables. As it is conventionally done, we postulate in our benchmark estimation that the observable variables are Home and Foreign output as well as Home and Foreign inflation.

The core of the NOEM model described in Table 1 would suggest that the trade-off between nominal 
and real variables can be articulated in terms of inflation and the output gaps (more specifically, between local inflation and a combination of Home and Foreign output gaps). However, the output gaps are not economic concepts that can be directly measured in the macro data as they require taking a stand on what potential output is and the answer to that is model-dependent. In order to complete the estimation model, therefore, we require a measurement equation that relates actual observed output to the output gap and its potential (described in Table 2). In other words, we are in fact estimating jointly a model for potential output together with the fully-fledged NOEM model that articulates the so-called 'global slack hypothesis'.

In our Bayesian estimation exercises, we distinguish between the power of these techniques to recover the true structural parameter (which we refer to as the strength of structural identification) and the actual economic content that is revealed a posteriori. We look at our results primarily from the perspective of the economic insight that we gain from applying the Bayesian estimation techniques, as the structural identification (the recovery of the true parameters) can be reasonably attained for all 13 parameters that we are estimating if the sample size is long enough and we rule out model misspecification. From this economic viewpoint, we take as given our belief about the structural parameters represented through probabilistic distributions, and define the goal of the estimation to be the extraction of all useful information from the available realization of observable macro aggregates to alter our prior belief about the model parameters. In this paper, we are taking the data generating process seriously in the terminology of Canova (1994), and using the very same time series that we are trying to explain in order to update our beliefs - something that runs counter to the conventional recommendations of the calibration approach as stated earlier.

Our benchmark estimation applied to the workhorse NOEM model described in this paper suggests that, given our priors, there is a number of model parameters for which their posterior distributions are going to be dominated by their priors. In other words, there is always a significant number of parameters for which the posterior distribution differs little from the prior distribution and, therefore, for which the economic insight gained from estimation is very limited. Some of those parameters are, in fact, crucial for the questions posited by the model - so Bayesian estimation becomes less useful in practice as a tool for economic thinking. The structural (policy and non-policy) parameters that present fairly similar prior and posterior distributions are $\sigma$ (which affects international risk-sharing through terms of trade fluctuations), $\xi$ (which defines the degree of trade openness in this environment), and $\psi_{\pi}$ (which describes the policy response to inflation). In turn, the posterior distributions change significantly for $\gamma$ and $\varphi$ (which enter into the slope of the Phillips curve), $\alpha$ (which indicates the degree of price stickiness), and $\psi_{x}$ (which describes the policy response to the output gap). For the shock parameters, however, only the persistence parameter of the monetary shocks, $\delta_{m}$, appears to change as we update our priors with the information extracted from the realization of the observables.

An important caveat to this is that, even though we maintain our priors invariant, the set of parameters for which economic insight can be gained from these estimation exercises varies depending on the set of observables. We elaborate further on this point later on. It is worth noticing that, among the structural parameters for which the gained economic insight from Bayesian estimation is marginal, we have the parameter $\xi$ that is tied in steady state to the import shares and is key for the international propagation of shocks. The other two parameters, $\sigma$ and $\psi_{\pi}$ cannot be linked to steady state relationships in the NOEM model, but their posterior distributions can be sensitive to the choice of the observable variables. We can include trade or interest rate variables in the set of observables for the purpose of estimating them with greater precision than in our priors. It is interesting to note that while the posterior distribution for $\psi_{x}$ is somewhat sensitive 
to the selection of observable variables, it is one notable case in which sample size matters a lot. While the prior and posterior distributions are close for the short sample of 160 periods, they both differ significantly as we increase the sample size (vastly enhancing the precision of our estimates). However, the same cannot be said for the other policy parameter $\psi_{\pi}$, as the posterior distributions do not vary much with different selection of observables or a larger sample size.

Finally, we confirm in all our exercises that it is possible to recover some of the persistence parameters with less dispersion around the mean than in their priors, while generally that is not the case for the parameters that characterize the variance-covariance matrix of the exogenous shocks. This has a direct effect on the perceived contribution of the structural shocks of the model to explain business cycle fluctuations and, in fact, it can be said that what we learn about the contribution of these shocks is very little relative to what our prior believes already dictated.

In most instances, we observe that Bayesian estimation is able to recover the true parameters, even for short samples of 160 observations. However, the presence of "Peso Problems" in short samples is vividly illustrated with the estimation of the third sub-sample (S3). We can see how in that case the posterior mean estimated for $\varphi$ is $40 \%$ higher than the true parameter, with little efficiency gain in the estimation of the other model parameters. This is not trivial, as $\varphi$ enters into the determination of the slope of the Phillips curve (Table 1) and affects the perceived trade-offs that monetary policy is facing. Generally, the set of parameters for which the posterior distribution differs from the prior distribution conforms with the principle that posterior dispersion is smaller than prior dispersion around the mean and declines with the sample size. This is most noticeable in the comparison between the posterior distribution derived from 160observation samples and 10,000-observation samples, or in the confidence intervals reported and summarized in the first block of Table 9. The posterior and prior distributions can be compared in Figures 6.A, 6.B, and 6.C.

\section{[Insert Table 9 and Figures 6.A, 6.B and 6.C about here.]}

The NOEM model we are investigating here does not incorporate deterministic or stochastic time trends explicitly. ${ }^{32}$ Hence, the question of pre-filtering in our model can be stated in terms of whether to demean or not to demean the data for estimation purposes. Three parameters are fundamental in that regard: the parameters $\kappa, A$ and $M$. These three parameters enter into the determination of the steady state, but they do not affect the short-run dynamics of the model. That was the primary reason why we chose to normalize them in the first place before simulating the model. These three parameters, however, have a direct effect on the steady state level of output in each country - as can be seen from Table 4-which are among our observable variables.

Looking at the population (theoretical) and sample means - in particular for the short samples of 160 periods - for Home and Foreign output in Table 7 it would be possible to argue that the steady state does not correspond to the actual value implied by the normalization that we have adopted for those three parameters. Therefore, the question arises as to the usefulness of pre-filtering (de-meaning) the data prior to estimation or not. Since the parameters that determine the steady state level of output do not affect the short-run dynamics, pre-filtering the data rather than augmenting the model with the steady state equations for Home and Foreign output to estimate those three parameters jointly with the rest of the model should not affect

\footnotetext{
${ }^{32}$ For a recent discussion on pre-filtering the data versus estimating the trend jointly with the rest of the model, see Ferroni (2011).
} 
the identification of the parameters we are really interested in. Hence, for simplicity, we conjecture that the normalization of the steady state output might be different and simply estimate the model after de-meaning the data. We do not attempt to extend the estimation to include those three normalized parameters and the steady state. However, our results reported in the second block of Table 9 suggest that the prefiltering does little to alter the results of the estimation or the precision of the estimation as implied by the posterior distributions of the model parameters - even for the short-samples of 160 quarters.

Selection of Observables. In order to avoid stochastic singularity, we must have at least as many shocks or measurement errors in the model as we have observed variables. Therefore, some of the key practical decisions that researchers have to make in using Bayesian estimation involve deciding: how many observables to estimate and, most importantly, which observables among all the available macro time series ought to be incorporated in the estimation. Here we consider a model characterized by only four structural shockswhich suggests that we need four observable variables - and experiment with the selection of alternative sets of four macro time series. In our benchmark implementation, we make inferences based on Home and Foreign output, as well as Home and Foreign inflation, and report the results in Table 9. Now we evaluate the model estimation findings when we replace Foreign output with either the terms of trade or the trade balance, while keeping the number of observables always at four. We summarize the results in Table 10 and with Figures 7.A, 7.B, and 7.C.

\section{[Insert Table 10 and Figures 7.A, 7.B and 7.C about here.]}

Looking at the estimation results for the long sample of 10, 000 observations including the terms of trade among the observables instead of foreign output, the most significant change we observe is in the estimation of the parameter $\sigma$. Those findings can be found in the first block of Table 10. This increased precision of the estimate can also be observed in the evidence reported for the shorter samples of 160 periods. The parameter $\sigma$ plays an important role in regulating trade flows and allowing international risk-sharing through fluctuations of the terms of trade; hence adding the terms of trade to the set of observables that we use has the natural advantage of helping us reveal further information about that parameter that we could not uncover using the set of observables in our benchmark implementation. We also observe that under this alternative implementation of our estimation the prior and posterior distributions for the parameter $\sigma$ differ greatly in Figures 7.A, 7.B, and 7.C, unlike what happens in our benchmark implementation as shown in Figures 6.A, 6.B, and 6.C. This is the most significant difference, but it indicates that the estimation results and what we learn from the data can depend on our data selection - an issue already noted, among others, by Guerron-Quintana (2010).

The second block of Table 10 reports the empirical results of the estimation for the case in which foreign output is replaced by the trade balance instead. The information content, however, is less relevant as we do not see a gain in the precision of our estimates of the parameter $\sigma$ relative to the benchmark implementationnot even for the full length sample of 10,000 periods. However, it is interesting to note from this exercise that 'peso problems' may appear anew for short samples, depending on the selection of variables that we use for estimation. For instance, we observe the case of sample S1, where the point estimates in the benchmark implementation are very close to their true values across all model parameters. If we estimate the same model with the trade balance as an observable in place of foreign output, we end up with a significantly biased estimate of the policy parameter $\psi_{\pi}$, which is a policy parameter that describes the sensitivity of 
the policy response to inflation. As we discussed earlier, this policy parameter is very important in order to quantify the real effects implied by the NOEM model, as a more aggressive policy stance should in general correspond with less inflation and smaller deviations from potential. ${ }^{33}$

The lesson we derive from these very simple exercises is that researchers must be mindful of these issues in the selection of observables for Bayesian estimation. Moreover, it is not obvious how to establish general guidelines on selecting observables that can be applied in every occasion. Common sense suggests that one may have to experiment with a number of possible combinations of observable variables and develop an understanding of the model that could help in the selection of observables (by pointing towards the data that can reveal the most useful information about the key structural parameters that the researcher is most interested to learn about). Having said that, under ideal circumstances the choice would solely depend on the type of question that the estimation exercise is meant to address. In practice, though, the data selection may be already significantly limited simply due to data availability limitations, and other data related issues that prevent us from using the kind of data that would be most relevant for our question. For example, in international macro economics, the quality and availability of time series macro data for emerging economies or the sample length of the data available and its quality are always a major handicap.

Inclusion of Non-Structural Shocks. We often see in applications of Bayesian estimation techniques that the set of observables is conveniently expanded with the addition of non-structural shocks. The ultimate goal of that is often to increase the information set that the Bayesian procedures can use to arguably help us refine our beliefs about the structural parameters of the model. One common practice is to increase the set of observables with the addition of measurement error shocks into the estimated model, which can also help capture the likely errors in the measurement of some of the variables themselves. The specification of those errors, however, is often rather ad hoc, and little motivation is given for the exogenous processes adscribed to these measurement errors. While this may be capturing important features of the data that is appropriate to take into account and serves to expand the set of observables, it might also introduce a misspecification problem. Here we consider the following case: one in which the true data observed is without error, but where we introduce in all our estimations a measurement error on either foreign output or the terms of trade. We model the measurement error as white noise with low volatility in hopes of keeping the distortion (or misspecification) from biasing our findings while taking advantage of the additional informational content of one more observable variable. In this case, we consider as our observables Home and Foreign output, Home and Foreign inflation, and the terms of trade.

[Insert Table 11 about here.]

The evidence is reported and summarized in Table 11, where the first block conducts the estimation under the assumption that the error term appears in measuring Foreign output while the second block assumes that it appears in the measurement of terms of trade. The most interesting finding is that we can obtain greater precision in the estimates of the parameter $\sigma$ from the inclusion of terms of trade among the set of observables, but only if the non-structural measurement error is added to the terms of trade equation and not to the foreign output equation. This is an interesting observation, because it clearly indicates that the

\footnotetext{
${ }^{33}$ In results not reported in the current draft, we also experimented replacing foreign output with a nominal variable such as home nominal interest rates. The interesting thing is that including interest rates in this way did not seem to help us gain more information about policy parameters than before, but it generally resulted in tighter estimates of the preference parameters $\varphi$ and $\gamma$ that are connected with the slope of the Phillips curve.
} 
addition of ad hoc non-structural shocks does not guarantee that more information will ultimately result into better estimates for the parameters we are interested in. We can also point out that 'Peso Problems' can be confounded with misspeficiation biases whenever we incorporate shocks arbitrarily (solely for the purpose of expanding the number of observables) in our estimated model. This may also occur depending on where we locate those measurement errors. We see, for instance, that the estimates of the volatility of the productivity shocks on the short sample of 160 observations (S2) vary significantly by a third depending on whether we have measurement errors placed on the foreign output equation or the terms of trade equation.

Setting up guidelines to deal with measurement errors is not a trivial matter. Our examples would suggest that it is preferable not to include measurement errors in the model solely for the sake of increasing the number of observables that we can use in our estimation exercise, as this may become a source of misspecification and introduce a bias in our estimation. It seems much more robust to add measurement errors when we have actual evidence that the data may be measured with error to help us both define the structure of the stochastic process that those non-structural shocks follow as well as to determine the proper measurement equations that would be affected in their presence. In any case, it seems plausible to suggest that the presence of measurement errors in the data may help us increase the number of observable variables that we can use for Bayesian estimation, but that does not necessarily mean we will necessarily end up with more information revealed through the posterior distributions of the key structural parameters of interest in our model.

\subsection{Other Insights}

As we have discussed extensively, the identification of the structural parameters given our priors about them through the implementation of conventional Bayesian techniques does not necessarily mean that the information revealed by the macro data is giving us greater economic insight about all of them. "Peso problems" will always be present when making inferences in small samples, and in those instances it truly matters the implementation strategy that the researcher follows (for example, regarding the pre-filtering of the data, the selection of observables or the inclusion of non-structural shocks as discussed before). One way in which we can assess the economic insight gained through Bayesian estimation is by looking at the Bayesian impulse response functions (IRFs) in relation to the theretical IRFs of the calibrated NOEM model that we are using as our data-generating process (DGP).

Figures 8.A, 8.B, 8.C and 8.D summarize those Bayesian IRFs for our benchmark implementation based on Home and Foreign output as well as Home and Foreign inflation as the observables. We easily conclude from those graphs that the posterior mean trajectory is often fairly well-aligned with the corresponding theoretical IRFs. However, the precision by which the model estimation can recover the true IRFs is dependent on the type of shock, and also dependent on the endogenous variable that is being shocked. For instance, the transmission of monetary shocks is less tightly estimated than the propagation of productivity shocks. The responses of terms of trade and inflation are also less precisely identified in this context. Naturally, we observe that the tightness of those estimated IRFs increases as we increase the sample size, but that (long sample size) is something that remains a luxury for international macroeconomists. It is interesting to point out, however, that consistently with our earlier discussion on the information content of terms of trade, we observe that in this benchmark implementation the estimation of the response of terms of trade to all the structural shocks remains significantly wider than that of other macro aggregates even 
when we are looking at a very long sample of 10,000 observations.

\section{[Insert Figures 8.A, 8.B, 8.C and 8.D about here.]}

Another important by-product of Bayesian estimation that we can exploit and investigate further in the context of small sample inference is the techniques of Bayesian model comparison and selection. In this case, we restrict ourselves to compare just two different model specifications: one is the Workhorse NOEM Model described in Tables 1 and 2, while the other is the Flexible Price (RBC) Model described in Table 3. We have already indicated that the optimal monetary policy within the NOEM framework would set inflation at zero in both countries, ensuring that the resulting allocation corresponds to the allocation under flexible prices (even though nominal rigidities are still present). Therefore, more aggressive policy responses to inflation should result - in general - in allocations that are increasingly more similar between the NOEM Model and the RBC Model, in turn, making it harder for the researcher to distinguish empirically between both of them based on observed macro data alone. Taking that into account, we use conventional posterior odds tests in Bayesian estimation to compare the two models. We keep the realization of the exogenous shocks invariant in each short sample of 160 periods and for the long sample of 10,000 periods for every comparison that we conduct, but we simulate the endogenous data that we will use for model comparison from the NOEM Model with nominal rigidities under different monetary policy regimes. Here, differences in the monetary policy regime correspond simply to differences in the aggressiveness of policy to inflation fluctuations.

All our results are summarized in Table 12, including up to five different monetary policy regimes of increasing anti-inflation aggressiveness. The first monetary policy regime corresponds to the benchmark monetary policy specification described in the calibration of the NOEM model. The priors are the same for the common parameters across the two models. Moreover, all prior distributions are kept invariant for estimation purposes in all exercises - except for the prior corresponding to the policy parameter $\psi_{\pi}$. In that case, we set the prior mean of the $\psi_{\pi}$ parameter to be equal to the true sensitivity of the monetary policy reaction function to inflation adopted, but we keep the standard deviation of the prior distribution equal to the one specified in the benchmark. We also specify a purely uninformative prior distribution on the two models we are comparing assigning half the probability to each one of them.

It is comforting to see that the posteriors odds test overwhelmingly favors the NOEM specification under the benchmark monetary policy regime, even for the case of the three short samples of 160 periods that we have investigated here. However, in all other comparison experiments with more aggressive monetary policy regimes, there is at least one subsample on which the $\mathrm{RBC}$ model is clearly favored or under which the posterior probability assigned to the RBC model is large. This empirical evidence in support of the RBC model naturally declines when we look at long samples, to the point that the evidence almost surely suggests the true DGP process is the one described by the NOEM model. Under these circumstances, however, it might be possible that our Bayesian estimates would end up supporting the RBC model under reasonably aggressive monetary policy regimes for shorter samples - even though the actual model specification behind the DGP process is the one corresponding to the NOEM Model with nominal rigidities.

This is another dimension of the so-called 'Peso Problem', as in short samples we might be inclined to prefer the wrong model. Also, it is an example of the practical limitations that researchers may encounter to successfully apply Bayesian model comparison techniques to recover the true model specification corresponding to the DGP of the data. The consequences could not be more severe on two grounds: First, because if we opt for the wrong model, then the structural parameter estimates themselves are subject to 
misspecification biases, which makes their economic interpretation more difficult; second, because the wrong model selection also gravely invalidates the policy analysis that can be conducted with the estimated model (as well as its forecasting ability). On the later point, it is important to recall that the crucial difference between the NOEM model and the RBC model is that one contemplates rigidities in the pricing behavior of firms that result in monetary non-neutrality, while the other (the RBC model) is one economic environment where monetary policy is neutral both in the long- and in the short-run. Therefore, if we were to conclude on the basis of the evidence available that the RBC model is favored by the data when it is actually the wrong model, then we may also conclude that a loosening of monetary policy will have no real effects on economic activity, when in reality it would have severe consequences that would only become obvious if the policy change were actually implemented and followed through.

[Insert Table 12 about here.]

\section{Concluding Remarks}

In this paper we have evaluated some of the challenges that researchers are faced with when attempting a structural evaluation of the global slack hypothesis. Working with the simplest possible specification of an open economy model that generates a short run relationship between global slack and domestic inflation we explore the problems assocaited with parameter identification and estimation in samples of the size typically available to international macroeconomists. We find that - even absent a problem of model misspecification - weak identification of key parameters is an issue in applying Bayesian techniques to these types of models. Using a convetional calibration of the basic model, we generate artificial data series which we then use to explore the ability of Bayesian techniques to recover the true parameter values. We find in our estimation with simulated data that identification is sensitive to the choice of observables and to the addition of non-structural shocks. We also show that misspecification can introduce severe biases and that model selection can be very difficult precisely when it is most needed (e.g., when monetary policy is near optimal). As a possible soloution to these problems in more complex model environmenst, we propose 'testing' the

model - as a preliminary step in Bayesian estimation - with simulated data to detect weak identification of key structural parameters. 


\section{Appendix}

\section{A Benchmark Open-Economy Model}

For tractability, we abstract from a number of relevant features like capital and investment (see, e.g., Chari et al. (2002), and Martínez-García and Søndergaard (2008)) and durable goods (see, e.g., Engel and Wang (2011)). We consider a two-country model with a continuum of unit mass of households and consumption varieties equally distributed between the Home country and the Foreign country. The structure of the model is a simplified version of the Martínez-García and Wynne (2010) with equal population sizes in both countries and local bias in consumption.

Households. The lifetime utility for a representative household in the Home country is additively separable in consumption, $C_{t}$, and labor, $L_{t}$, i.e.,

$$
\sum_{\tau=0}^{+\infty} \beta^{\tau} \mathbb{E}_{t}\left[\frac{1}{1-\gamma}\left(C_{t+\tau}\right)^{1-\gamma}-\frac{\kappa}{1+\varphi}\left(L_{t+\tau}\right)^{1+\varphi}\right]
$$

where $0<\beta<1$ is the subjective intertemporal discount factor. The inverse of the intertemporal elasticity of substitution and the inverse of the Frisch elasticity of labor supply satisfy that $\gamma>0$ and $\varphi>0$, respectively. The scaling factor $\kappa$ must be nonnegative. The Home household maximizes its lifetime utility subject to the sequence of budget constraints,

$$
P_{t} C_{t}+\int_{\omega_{t+1} \in \Omega} Q_{t}\left(\omega_{t+1}\right) B_{t}\left(\omega_{t+1}\right) \leq B_{t-1}\left(\omega_{t}\right)+W_{t} L_{t}+P r_{t}-T_{t}
$$

where $W_{t}$ is the nominal wage in the Home country, $P_{t}$ is the Home consumption price index $(\mathrm{CPI}), S_{t}$ is the nominal exchange rate, $T_{t}$ is the nominal lump-sum tax (or transfer) from the Home government, and $\operatorname{Pr}_{t}$ are (per-period) nominal profits from all firms producing the Home varieties. We assume a set of one-period Arrow-Debreu securities (contingent bonds) internationally traded and in zero net supply, $B_{t}\left(\omega_{t+1}\right)$. For simplicity, these contingent bonds are quoted in the units of account of the Home country. The Home price of the contingent bonds is denoted $Q_{t}\left(\omega_{t+1}\right)$, while the Foreign price is $Q_{t}^{*}\left(\omega_{t+1}\right)=\frac{1}{S_{t}} Q_{t}\left(\omega_{t+1}\right)$. Similarly, for the representative household in the Foreign country. The existence of a full set of one-period Arrow-Debreu securities suffices to complete the local and international asset markets recursively.

Under complete asset markets, households can perfectly share risks internationally and the intertemporal marginal rate of substitution is equalized across countries in every state of nature,

$$
\beta\left(\frac{C_{t+1}}{C_{t}}\right)^{-\gamma} \frac{P_{t}}{P_{t+1}}=\beta\left(\frac{C_{t+1}^{*}}{C_{t}^{*}}\right)^{-\gamma} \frac{P_{t}^{*} S_{t}}{P_{t+1}^{*} S_{t+1}},
$$

where $P_{t}^{*}$ is the Foreign CPI and $C_{t}^{*}$ stands for Foreign consumption. We define the real exchange rate as $R S_{t} \equiv \frac{S_{t} P_{t}^{*}}{P_{t}}$, so by backward recursion we can express this perfect international risk-sharing condition as,

$$
R S_{t}=v\left(\frac{C_{t}^{*}}{C_{t}}\right)^{-\gamma}
$$


where $v \equiv \frac{S_{0} P_{0}^{*}}{P_{0}}\left(\frac{C_{0}^{*}}{C_{0}}\right)^{\gamma}$ is a constant that depends on the initial conditions. ${ }^{34}$ We can also price a redundant one-period, uncontingent nominal bond with the price of the contigent Arrow-Debreu securities and obtain a conventional set of stochastic Euler equations for both countries,

$$
\begin{aligned}
& \frac{1}{1+i_{t}}=\beta \mathbb{E}_{t}\left[\left(\frac{C_{t+1}}{C_{t}}\right)^{-\gamma} \frac{P_{t}}{P_{t+1}}\right], \\
& \frac{1}{1+i_{t}^{*}}=\beta \mathbb{E}_{t}\left[\left(\frac{C_{t+1}^{*}}{C_{t}^{*}}\right)^{-\gamma} \frac{P_{t}^{*}}{P_{t+1}^{*}}\right],
\end{aligned}
$$

where $i_{t}$ is the riskless, nominal interest rate in the Home country and $i_{t}^{*}$ is its Foreign country counterpart. The households' optimization problem also defines a pair of labor supply equations which can be expressed as,

$$
\begin{aligned}
\frac{W_{t}}{P_{t}} & =\kappa\left(C_{t}\right)^{\gamma}\left(L_{t}\right)^{\varphi}, \\
\frac{W_{t}^{*}}{P_{t}^{*}} & =\kappa\left(C_{t}^{*}\right)^{\gamma}\left(L_{t}^{*}\right)^{\varphi},
\end{aligned}
$$

plus the appropriate no-Ponzi games, transversality conditions and the budget constraint of both representative households.

$C_{t}$ is a CES aggregator of Home and Foreign goods for a representative Home country household defined as,

$$
C_{t}=\left[\xi^{\frac{1}{\sigma}}\left(C_{t}^{H}\right)^{\frac{\sigma-1}{\sigma}}+(1-\xi)^{\frac{1}{\sigma}}\left(C_{t}^{F}\right)^{\frac{\sigma-1}{\sigma}}\right]
$$

where $\sigma>0$ is the elasticity of substitution between the Home-produced and the Foreign-produced consumption bundles $C_{t}^{H}$ and $C_{t}^{F}$. The share of Home-produced goods in the Home consumption basket must satisfy that $\frac{1}{2} \leq \xi<1$. Analogous preferences are assumed for the Foreign country representative household, except that $C_{t}^{*}$ is defined as a CES aggregator of Home and Foreign goods in the following terms,

$$
C_{t}^{*}=\left[\left(\xi^{*}\right)^{\frac{1}{\sigma}}\left(C_{t}^{H *}\right)^{\frac{\sigma-1}{\sigma}}+\left(1-\xi^{*}\right)^{\frac{1}{\sigma}}\left(C_{t}^{F *}\right)^{\frac{\sigma-1}{\sigma}}\right]
$$

where the share of Foreign-produced goods in the Foreign consumption basket must satisfy that $\left(1-\xi^{*}\right)=\xi$ ensuring symmetry while allowing the consumption basket to differ across countries (whenever $\xi \neq \frac{1}{2}$ ).

The sub-indexes $C_{t}^{H}$ and $C_{t}^{H *}$ indicate respectively Home and Foreign consumption of the bundle of differentiated variaties produced in the Home country. Similarly, $C_{t}^{F}$ and $C_{t}^{F *}$ denote Home and Foreign consumption of the bundle of differentiated varieties produced in the Foreign country. These sub-indexes

\footnotetext{
${ }^{34}$ In a symmetric steady state, the constant $v$ in the above expression is equal to one assuming the initial conditions are equated with the steady state.
} 
are defined as follows,

$$
\begin{aligned}
C_{t}^{H} & =\left[\left(\frac{1}{2}\right)^{-\frac{1}{\theta}} \int_{0}^{\frac{1}{2}} C_{t}(h)^{\frac{\theta-1}{\theta}} d h\right]^{\frac{\theta}{\theta-1}}, C_{t}^{F}=\left[\left(\frac{1}{2}\right)^{-\frac{1}{\theta}} \int_{\frac{1}{2}}^{1} C_{t}(f)^{\frac{\theta-1}{\theta}} d f\right]^{\frac{\theta}{\theta-1}}, \\
C_{t}^{H *} & =\left[\left(\frac{1}{2}\right)^{-\frac{1}{\theta}} \int_{0}^{\frac{1}{2}} C_{t}^{*}(h)^{\frac{\theta-1}{\theta}} d h\right]^{\frac{\theta}{\theta-1}}, C_{t}^{F *}=\left[\left(\frac{1}{2}\right)^{-\frac{1}{\theta}} \int_{\frac{1}{2}}^{1} C_{t}^{*}(f)^{\frac{\theta-1}{\theta}} d f\right]^{\frac{\theta}{\theta-1}},
\end{aligned}
$$

where $\theta>1$ is the elasticity of substitution across differentiated varieties within a country. Similarly, per-household total output and labor are expressed as,

$$
\begin{aligned}
& \frac{1}{2} Y_{t}=\left[\left(\frac{1}{2}\right)^{-\frac{1}{\theta}} \int_{0}^{\frac{1}{2}} Y_{t}(h)^{\frac{\theta-1}{\theta}} d h\right]^{\frac{\theta}{\theta-1}}, \frac{1}{2} Y_{t}^{*}=\left[\left(\frac{1}{2}\right)^{-\frac{1}{\theta}} \int_{\frac{1}{2}}^{1} Y_{t}^{*}(f)^{\frac{\theta-1}{\theta}} d f\right]^{\frac{\theta}{\theta-1}}, \\
& \frac{1}{2} L_{t}=\left[\left(\frac{1}{2}\right)^{-\frac{1}{\theta}} \int_{0}^{\frac{1}{2}} L_{t}(h)^{\frac{\theta-1}{\theta}} d h\right]^{\frac{\theta}{\theta-1}}, \frac{1}{2} L_{t}^{*}=\left[\left(\frac{1}{2}\right)^{-\frac{1}{\theta}} \int_{\frac{1}{2}}^{1} L_{t}^{*}(f)^{\frac{\theta-1}{\theta}} d f\right]^{\frac{\theta}{\theta-1}}
\end{aligned}
$$

where $Y_{t}$ and $Y_{t}^{*}$ denote the total output per household produced by firms in the Home and Foreign countries respectively, while $L_{t}$ and $L_{t}^{*}$ refer to the per-household total labor employed. The consumption-based price indexes which correspond to this specification of consumption preferences are,

$$
\begin{aligned}
P_{t} & =\left[\xi\left(P_{t}^{H}\right)^{1-\sigma}+(1-\xi)\left(P_{t}^{F}\right)^{1-\sigma}\right]^{\frac{1}{1-\sigma}} \\
P_{t}^{*} & =\left[\xi^{*}\left(P_{t}^{H *}\right)^{1-\sigma}+\left(1-\xi^{*}\right)\left(P_{t}^{F *}\right)^{1-\sigma}\right]^{\frac{1}{1-\sigma}}=\left[(1-\xi)\left(P_{t}^{H *}\right)^{1-\sigma}+\xi\left(P_{t}^{F *}\right)^{1-\sigma}\right]^{\frac{1}{1-\sigma}}
\end{aligned}
$$

and,

$$
\begin{aligned}
P_{t}^{H} & =\left[2 \int_{0}^{\frac{1}{2}} P_{t}(h)^{1-\theta} d h\right]^{\frac{1}{1-\theta}}, P_{t}^{F}=\left[2 \int_{\frac{1}{2}}^{1} P_{t}(f)^{1-\theta} d f\right]^{\frac{1}{1-\theta}}, \\
P_{t}^{H *} & =\left[2 \int_{0}^{\frac{1}{2}} P_{t}^{*}(h)^{1-\theta} d h\right]^{\frac{1}{1-\theta}}, P_{t}^{F *}=\left[2 \int_{\frac{1}{2}}^{1} P_{t}^{*}(f)^{1-\theta} d f\right]^{\frac{1}{1-\theta}},
\end{aligned}
$$

where $P_{t}^{H}$ and $P_{t}^{F}$ are the price sub-indexes for the Home-produced and Foreign-produced bundles of varieties in the Home market. The Home and Foreign price of the Home-produced variety $h$ is given by $P_{t}(h)$ and $P_{t}^{*}(h)$, respectively. Similarly for the sub-indexes $P_{t}^{H *}$ and $P_{t}^{F *}$ in the Foreign market and the prices of the Foreign-produced variety $f$ given by $P_{t}(f)$ and $P_{t}^{*}(f)$.

Firms. Each firm supplies the Home and Foreign markets under monopolistic competition in its own variety. Each firm produces only one differentiated variety, so there is a mass one of firms equally-distributed between the two countries. We assume producer currency pricing (PCP), so firms set Home and Foreign prices invoicing local sales and exports in their local currency. The PCP assumption implies complete exchange rate pass-through and also that the law of one price (LOOP) holds (i.e. $P_{t}(h)=S_{t} P_{t}^{*}(h)$ and $\left.P_{t}(f)=S_{t} P_{t}^{*}(f)\right)$. So it follows that $P_{t}^{H}=S_{t} P_{t}^{H *}$ and $P_{t}^{F}=S_{t} P_{t}^{F *}$. However, the assumption of Homeproduct bias in consumption leads to different consumption-based price indexes across countries and to 
deviations from purchasing power parity (PPP) in the model whenever $\xi \neq \frac{1}{2}$. For this reason, $P_{t} \neq S_{t} P_{t}^{*}$ and the real exchange rate becomes $R S_{t} \equiv \frac{S_{t} P_{t}^{*}}{P_{t}} \neq 1$.

Given the households' preferences, we can derive the demand for any Home variety $h$ and for any Foreign variety $f$ as,

$$
\begin{aligned}
& Y_{t}(h)=\frac{1}{2} C_{t}(h)+\frac{1}{2} C_{t}^{*}(h)=\left(\frac{P_{t}(h)}{P_{t}^{H}}\right)^{-\theta}\left\{\left(\frac{P_{t}^{H}}{P_{t}}\right)^{-\sigma}\left[\xi C_{t}+(1-\xi)\left(\frac{1}{R S_{t}}\right)^{-\sigma} C_{t}^{*}\right]\right\} \text {, if } h \in\left[0, \frac{1}{2}\right\} \text { 4) } \\
& Y_{t}^{*}(f)=\frac{1}{2} C_{t}(f)+\frac{1}{2} C_{t}^{*}(f)=\left(\frac{P_{t}(f)}{P_{t}^{F}}\right)^{-\theta}\left\{\left(\frac{P_{t}^{F}}{P_{t}}\right)^{-\sigma}\left[(1-\xi) C_{t}+\xi\left(\frac{1}{R S_{t}}\right)^{-\sigma} C_{t}^{*}\right]\right\}, \text { if } f \in\left(\frac{1}{2},(2) .5\right)
\end{aligned}
$$

Movements in the real exchange rate affect the total demand of all Home and Foreign varieties. Firms know the form of the individual demand functions given by (24) and (25), and produce their own varieties subject to a linear-in-labor technology. We impose competitive national labor markets, homogeneity of the labor input and labor immobility across countries, so wages equalize within a country. We assume that the local governments raises lump-sum taxes from households in order to subsize their local labor market, and define the (before-subsidy) nominal marginal cost in both countries as,

$$
M C_{t} \equiv\left(\frac{W_{t}}{A_{t}}\right), M C_{t}^{*} \equiv\left(\frac{W_{t}^{*}}{A_{t}^{*}}\right)
$$

where $W_{t}$ and $W_{t}^{*}$ are the Home and Foreign nominal wages. Home and Foreign productivity shocks are denoted by $A_{t}$ and $A_{t}^{*}$ respectively.

We model frictions in the goods market with firms maximizing profits subject to a partial adjustment rule à la Calvo (1983) on nominal prices at the variety level. In each period, every given firm receives with probability $0<\alpha<1$ a signal to maintain their prices and with probability $1-\alpha$ a signal to re-optimize. The fraction of re-optimizing Home firms in every period chooses a price $\widetilde{P}_{t}(h)$ optimally by maximizing the expected discounted value of their profits, i.e.,

$$
\sum_{\tau=0}^{+\infty} \mathbb{E}_{t}\left\{(\alpha \beta)^{\tau}\left(\frac{C_{t+\tau}}{C_{t}}\right)^{-\gamma} \frac{P_{t}}{P_{t+\tau}}\left[\widetilde{Y}_{t, t+\tau}(h)\left(\widetilde{P}_{t}(h)-(1-\phi) M C_{t+\tau}\right)\right]\right\}
$$

subject to the constraint of always satisfying demand at the chosen price $\widetilde{P}_{t}(h)$ as long as those prices remain unchanged. $\widetilde{Y}_{t, t+\tau}(h)$ indicates the total consumption demand of variety $h$ at time $t+\tau$ whenever the prevailing prices are unchanged since time $t$, i.e. whenever $P_{t+\tau}(h)=\widetilde{P}_{t}(h)$. We introduce the labor subsidy as proportional to the nominal marginal cost, with the proportion $\phi$ being time-invariant. Similarly, we describe the problem of the re-optimizing Foreign firms and define the optimal price $\widetilde{P}_{t}^{*}(f)$ and the corresponding demand schedule $\widetilde{Y}_{t, t+\tau}^{*}(f)$.

Therefore, the optimal choice of re-optimizing Home firms at time $t$ is given by,

$$
\widetilde{P}_{t}(h)=\left(\frac{\theta}{\theta-1}(1-\phi)\right) \frac{\sum_{\tau=0}^{+\infty}(\alpha \beta)^{\tau} \mathbb{E}_{t}\left[\left(\frac{C_{t+\tau}^{-\gamma}}{P_{t+\tau}}\right) \widetilde{Y}_{t, t+\tau}(h) M C_{t+\tau}\right]}{\sum_{\tau=0}^{+\infty}(\alpha \beta)^{\tau} \mathbb{E}_{t}\left[\left(\frac{C_{t+\tau}^{-\gamma}}{P_{t+\tau}}\right) \widetilde{Y}_{t, t+\tau}(h)\right]},
$$


and the optimal choice of re-optimizing Foreign firms is,

$$
\widetilde{P}_{t}^{*}(f)=\left(\frac{\theta}{\theta-1}(1-\phi)\right) \frac{\sum_{\tau=0}^{+\infty}(\alpha \beta)^{\tau} \mathbb{E}_{t}\left[\left(\frac{C_{t+\tau}^{*-\gamma}}{P_{t+\tau}^{*}}\right) \tilde{Y}_{t, t+\tau}^{*}(f) M C_{t+\tau}^{*}\right]}{\sum_{\tau=0}^{+\infty}(\alpha \beta)^{\tau} \mathbb{E}_{t}\left[\left(\frac{C_{t+\tau}^{*-\gamma}}{P_{t+\tau}^{*}}\right) \tilde{Y}_{t, t+\tau}^{*}(f)\right]},
$$

Monopolistic competition in production introduces a mark-up between prices and marginal costs, $\frac{\theta}{\theta-1}$, which is a function of the elasticity of substitution across varieties within a country $\theta>1$. We choose an optimal labor subsidy $\phi=\frac{1}{\theta}$ in both countries to neutralize this mark-up wedge, fully funded by lump-sum taxes raised on the local households. Given the inherent symmetry of the Calvo-type pricing scheme, the price sub-indexes $P_{t}^{H}$ and evolves according to the following pair of equations,

$$
\begin{aligned}
\left(P_{t}^{H}\right)^{1-\theta} & =\alpha\left(P_{t-1}^{H}\right)^{1-\theta}+(1-\alpha)\left(\widetilde{P}_{t}(h)\right)^{1-\theta}, \\
\left(P_{t}^{F *}\right)^{1-\theta} & =\alpha\left(P_{t-1}^{F *}\right)^{1-\theta}+(1-\alpha)\left(\widetilde{P}_{t}^{*}(f)\right)^{1-\theta} .
\end{aligned}
$$

The other pair of price sub-indexes, $P_{t}^{H *}$ and $P_{t}^{F}$, follow from these two and the LOOP condition.

Monetary Policy. We assume that the monetary authorities of the Home and Foreign countries set short term nominal interest rates, $i_{t}$ and $i_{t}^{*}$, according to Taylor (1993) type rules,

$$
\begin{aligned}
1+i_{t} & =(1+\bar{i}) M_{t}\left[\left(\frac{\Pi_{t}}{\bar{\Pi}}\right)^{\psi_{\pi}}\left(\frac{Y_{t}}{\bar{Y}_{t}}\right)^{\psi_{x}}\right], \\
1+i_{t}^{*} & =\left(1+\bar{i}^{*}\right) M_{t}^{*}\left[\left(\frac{\Pi_{t}^{*}}{\bar{\Pi}^{*}}\right)^{\psi_{\pi}}\left(\frac{Y_{t}^{*}}{\bar{Y}_{t}^{*}}\right)^{\psi_{x}}\right],
\end{aligned}
$$

where $M_{t}$ and $M_{t}^{*}$ are the Home and Foreign monetary policy shocks, $\bar{i}$ and $\bar{i}^{*}$ are the Home and Foreign nominal interest rates, and $\psi_{\pi}>1$ and $\psi_{x}>0$ represent the sensitivity of the monetary policy rule to movements in inflation and the output gap respectively. $\Pi_{t} \equiv \frac{P_{t}}{P_{t-1}}$ and $\Pi_{t}^{*} \equiv \frac{P_{t}^{*}}{P_{t-1}^{*}}$ are the (gross) CPI inflation rates, while $\bar{\Pi}$ and $\bar{\Pi}^{*}$ are the corresponding steady state inflation rates that are targeted by the national monetary authorities. $Y_{t}$ and $Y_{t}^{*}$ define the per capita output levels, while $\bar{Y}_{t}$ and $\bar{Y}_{t}^{*}$ are the potential per capita output levels that monetary policy tracks. The ratios $\frac{Y_{t}}{\bar{Y}_{t}}$ and $\frac{Y_{t}^{*}}{\bar{Y}_{t}^{*}}$ define the output gaps in levels for the Home and Foreign country. Potential per capita output is aggregated in the same way as per capita output, but it is defined as the output level that would prevail in the economy if all frictions could be eliminated - that is, in a frictionless economy with competitive firms and flexible prices. This index specification of the monetary policy rules take the standard form postulated by Taylor (1993) once they are log-linearized. 


\section{B Proofs}

Proof of Proposition 1. The potential output of both countries can be expressed as a linear transformation of the productivity shocks in the following terms,

$$
\left(\begin{array}{c}
\widehat{\bar{y}}_{t} \\
\widehat{\bar{y}}_{t}^{*}
\end{array}\right) \approx\left(\frac{1+\varphi}{\gamma+\varphi}\right)\left(\begin{array}{cc}
\Lambda & (1-\Lambda) \\
(1-\Lambda) & \Lambda
\end{array}\right)\left(\begin{array}{c}
\widehat{a}_{t} \\
\widehat{a}_{t}^{*}
\end{array}\right) .
$$

Assuming invertibility, the vector of potential output inherits the $V A R(1)$ stochastic structure of the productivity shocks. Accordingly, the potential output process takes the following stochastic form,

$$
\begin{aligned}
& \left(\begin{array}{c}
\widehat{\bar{y}}_{t} \\
\widehat{\bar{y}}_{t}^{*}
\end{array}\right) \approx\left(\begin{array}{cc}
\Lambda & 1-\Lambda \\
1-\Lambda & \Lambda
\end{array}\right)\left(\begin{array}{cc}
\delta_{a} & \delta_{a, a^{*}} \\
\delta_{a, a^{*}} & \delta_{a}
\end{array}\right)\left(\begin{array}{cc}
\Lambda & 1-\Lambda \\
1-\Lambda & \Lambda
\end{array}\right)^{-1}\left(\begin{array}{c}
\widehat{\bar{y}}_{t-1} \\
\widehat{\bar{y}}_{t-1}^{*}
\end{array}\right)+\left(\frac{1+\varphi}{\gamma+\varphi}\right)\left(\begin{array}{cc}
\Lambda & 1-\Lambda \\
1-\Lambda & \Lambda
\end{array}\right)\left(\begin{array}{c}
\widehat{\varepsilon}_{t}^{a} \\
\widehat{\varepsilon}_{t}^{a *}
\end{array}\right), \\
& \left(\begin{array}{c}
\widehat{\varepsilon}_{t}^{a} \\
\widehat{\varepsilon}_{t}^{a *}
\end{array}\right) \sim N\left(\left(\begin{array}{l}
0 \\
0
\end{array}\right),\left(\begin{array}{cc}
\sigma_{a}^{2} & \rho_{a, a^{*}} \sigma_{a}^{2} \\
\rho_{a, a^{*}} \sigma_{a}^{2} & \sigma_{a}^{2}
\end{array}\right)\right) .
\end{aligned}
$$

where,

$$
\left(\begin{array}{cc}
\Lambda & 1-\Lambda \\
1-\Lambda & \Lambda
\end{array}\right)^{-1}=\frac{1}{2 \Lambda-1}\left(\begin{array}{cc}
\Lambda & \Lambda-1 \\
\Lambda-1 & \Lambda
\end{array}\right) .
$$

Hence, it follows from here that,

$$
\begin{aligned}
& \left(\begin{array}{cc}
\Lambda & 1-\Lambda \\
1-\Lambda & \Lambda
\end{array}\right)\left(\begin{array}{cc}
\delta_{a} & \delta_{a, a^{*}} \\
\delta_{a, a^{*}} & \delta_{a}
\end{array}\right)\left(\begin{array}{cc}
\Lambda & 1-\Lambda \\
1-\Lambda & \Lambda
\end{array}\right) \\
& =\frac{1}{2 \Lambda-1}\left(\begin{array}{cc}
\Lambda & 1-\Lambda \\
1-\Lambda & \Lambda
\end{array}\right)\left(\begin{array}{cc}
\delta_{a} & \delta_{a, a^{*}} \\
\delta_{a, a^{*}} & \delta_{a}
\end{array}\right)\left(\begin{array}{cc}
\Lambda & \Lambda-1 \\
\Lambda-1 & \Lambda
\end{array}\right) \\
& =\left(\begin{array}{cc}
\delta_{a} & \delta_{a, a^{*}} \\
\delta_{a, a^{*}} & \delta_{a}
\end{array}\right)
\end{aligned}
$$

which implies that the structure of the $V A R(1)$ for the potential output inherits the persistence structure of the underlying productivity shocks. Moreover, we can simplify the notation by expressing the innovations to the output potential process in the following terms,

$$
\begin{aligned}
\left(\begin{array}{c}
\widehat{\varepsilon}_{t}^{r} \\
\widehat{\varepsilon}_{t}^{r *}
\end{array}\right) & \equiv\left(\frac{1+\varphi}{\gamma+\varphi}\right)\left(\begin{array}{cc}
\Lambda & 1-\Lambda \\
1-\Lambda & \Lambda
\end{array}\right)\left(\begin{array}{c}
\widehat{\varepsilon}_{t}^{a} \\
\widehat{\varepsilon}_{t}^{a *}
\end{array}\right), \\
\left(\begin{array}{c}
\widehat{\varepsilon}_{t}^{r} \\
\widehat{\varepsilon}_{t}^{r *}
\end{array}\right) & \sim N\left(\left(\begin{array}{c}
0 \\
0
\end{array}\right),\left(\frac{1+\varphi}{\gamma+\varphi}\right)^{2}\left(\begin{array}{cc}
\Lambda & 1-\Lambda \\
1-\Lambda & \Lambda
\end{array}\right)\left(\begin{array}{cc}
\sigma_{a}^{2} & \rho_{a, a^{*}} \sigma_{a}^{2} \\
\rho_{a, a^{*}} \sigma_{a}^{2} & \sigma_{a}^{2}
\end{array}\right)\left(\begin{array}{cc}
\Lambda & 1-\Lambda \\
1-\Lambda & \Lambda
\end{array}\right)^{T}\right),
\end{aligned}
$$


where,

$$
\begin{aligned}
& \left(\frac{1+\varphi}{\gamma+\varphi}\right)^{2}\left(\begin{array}{cc}
\Lambda & 1-\Lambda \\
1-\Lambda & \Lambda
\end{array}\right)\left(\begin{array}{cc}
\sigma_{a}^{2} & \rho_{a, a^{*}} \sigma_{a}^{2} \\
\rho_{a, a^{*}} \sigma_{a}^{2} & \sigma_{a}^{2}
\end{array}\right)\left(\begin{array}{cc}
\Lambda & 1-\Lambda \\
1-\Lambda & \Lambda
\end{array}\right)^{T} \\
& =\left(\frac{1+\varphi}{\gamma+\varphi}\right)^{2}\left(\begin{array}{cc}
\Lambda & 1-\Lambda \\
1-\Lambda & \Lambda
\end{array}\right)\left(\begin{array}{cc}
\sigma_{a}^{2} & \rho_{a, a^{*}} \sigma_{a}^{2} \\
\rho_{a, a^{*}} \sigma_{a}^{2} & \sigma_{a}^{2}
\end{array}\right)\left(\begin{array}{cc}
\Lambda & 1-\Lambda \\
1-\Lambda & \Lambda
\end{array}\right) \\
& =\sigma_{a}^{2}\left(\frac{1+\varphi}{\gamma+\varphi}\right)^{2}\left(\begin{array}{cc}
(\Lambda)^{2}+2 \rho_{a, a^{*}} \Lambda(1-\Lambda)+(1-\Lambda)^{2} & \rho_{a, a^{*}}(\Lambda)^{2}+2 \Lambda(1-\Lambda)+\rho_{a, a^{*}}(1-\Lambda)^{2} \\
\rho_{a, a^{*}}(\Lambda)^{2}+2 \Lambda(1-\Lambda)+\rho_{a, a^{*}}(1-\Lambda)^{2} & (\Lambda)^{2}+2 \rho_{a, a^{*}} \Lambda(1-\Lambda)+(1-\Lambda)^{2}
\end{array}\right) \\
& =\sigma_{a}^{2}\left(\frac{1+\varphi}{\gamma+\varphi}\right)^{2}\left((\Lambda)^{2}+2 \rho_{a, a^{*}} \Lambda(1-\Lambda)+(1-\Lambda)^{2}\right)\left(\begin{array}{cc}
1 & \frac{\rho_{a, a^{*}}(\Lambda)^{2}+2 \Lambda(1-\Lambda)+\rho_{a, a^{*}}(1-\Lambda)^{2}}{(\Lambda)^{2}+2 \rho_{a, a^{*}} \Lambda(1-\Lambda)+(1-\Lambda)^{2}} \\
\frac{\rho_{a, a^{*}}(\Lambda)^{2}+2 \Lambda(1-\Lambda)+\rho_{a, a^{*}}(1-\Lambda)^{2}}{(\Lambda)^{2}+2 \rho_{a, a^{*}} \Lambda(1-\Lambda)+(1-\Lambda)^{2}} & 1
\end{array}\right.
\end{aligned}
$$

Hence, we define the volatility and the correlation of the output potential innovations in the following fashion,

$$
\begin{aligned}
\sigma_{y}^{2} & =\sigma_{a}^{2}\left(\frac{1+\varphi}{\gamma+\varphi}\right)^{2}\left((\Lambda)^{2}+2 \rho_{a, a^{*}} \Lambda(1-\Lambda)+(1-\Lambda)^{2}\right), \\
\rho_{y, y^{*}} & =\frac{\rho_{a, a^{*}}(\Lambda)^{2}+2 \Lambda(1-\Lambda)+\rho_{a, a^{*}}(1-\Lambda)^{2}}{(\Lambda)^{2}+2 \rho_{a, a^{*}} \Lambda(1-\Lambda)+(1-\Lambda)^{2}}
\end{aligned}
$$

Proof of Corollary 1. The natural rates of both countries can be expressed as a linear transformation of the productivity shocks in the following terms,

$$
\begin{aligned}
\left(\begin{array}{c}
\widehat{\bar{r}}_{t} \\
\widehat{\bar{r}}_{t}^{*}
\end{array}\right) & \approx \gamma\left(\frac{1+\varphi}{\gamma+\varphi}\right)\left(\begin{array}{cc}
(\Theta \Lambda+(1-\Theta)(1-\Lambda)) & (1-(\Theta \Lambda+(1-\Theta)(1-\Lambda))) \\
(1-(\Theta \Lambda+(1-\Theta)(1-\Lambda))) & (\Theta \Lambda+(1-\Theta)(1-\Lambda))
\end{array}\right)\left(\begin{array}{c}
\mathbb{E}_{t}\left[\Delta \widehat{a}_{t+1}\right] \\
\mathbb{E}_{t}\left[\Delta \widehat{a}_{t+1}^{*}\right]
\end{array}\right) \\
& \approx \gamma\left(\frac{1+\varphi}{\gamma+\varphi}\right)\left(\begin{array}{cc}
(\Theta \Lambda+(1-\Theta)(1-\Lambda)) & (1-(\Theta \Lambda+(1-\Theta)(1-\Lambda))) \\
(1-(\Theta \Lambda+(1-\Theta)(1-\Lambda))) & (\Theta \Lambda+(1-\Theta)(1-\Lambda))
\end{array}\right)\left(\begin{array}{cc}
\delta_{a}-1 & \delta_{a, a^{*}} \\
\delta_{a, a^{*}} & \delta_{a}-1
\end{array}\right)\left(\begin{array}{c}
\widehat{a}_{t} \\
\widehat{a}_{t}^{*}
\end{array}\right) \\
& \approx \gamma\left(\frac{1+\varphi}{\gamma+\varphi}\right)\left(\begin{array}{cc}
\Pi_{1} & \Pi_{2} \\
\Pi_{2} & \Pi_{1}
\end{array}\right)\left(\begin{array}{c}
\widehat{a}_{t} \\
\widehat{a}_{t}^{*}
\end{array}\right),
\end{aligned}
$$

where,

$$
\begin{aligned}
\Theta \Lambda & +(1-\Theta)(1-\Lambda)=\xi\left(\frac{\varphi(\sigma \gamma-(\sigma \gamma-1)(2 \xi-1))+\gamma}{\varphi\left(\sigma \gamma-(\sigma \gamma-1)(2 \xi-1)^{2}\right)+\gamma}\right), \\
\Pi_{1} & \equiv(\Theta \Lambda+(1-\Theta)(1-\Lambda))\left(\delta_{a}-1\right)+(1-(\Theta \Lambda+(1-\Theta)(1-\Lambda))) \delta_{a, a^{*}} \\
& =\delta_{a, a^{*}}-\xi\left(\frac{\varphi(\sigma \gamma-(\sigma \gamma-1)(2 \xi-1))+\gamma}{\varphi\left(\sigma \gamma-(\sigma \gamma-1)(2 \xi-1)^{2}\right)+\gamma}\right)\left(1+\delta_{a, a^{*}}-\delta_{a}\right), \\
\Pi_{2} & \equiv(\Theta \Lambda+(1-\Theta)(1-\Lambda)) \delta_{a, a^{*}}+(1-(\Theta \Lambda+(1-\Theta)(1-\Lambda)))\left(\delta_{a}-1\right) \\
& =\left(\delta_{a}-1\right)+\xi\left(\frac{\varphi(\sigma \gamma-(\sigma \gamma-1)(2 \xi-1))+\gamma}{\varphi\left(\sigma \gamma-(\sigma \gamma-1)(2 \xi-1)^{2}\right)+\gamma}\right)\left(1+\delta_{a, a^{*}}-\delta_{a}\right) .
\end{aligned}
$$

Assuming invertibility, the vector of natural rates inherits the $V A R(1)$ stochastic structure of the produc- 
tivity shocks. Accordingly, the natural rates take the following stochastic form,

$$
\begin{aligned}
\left(\begin{array}{c}
\widehat{\bar{r}}_{t} \\
\widehat{\bar{r}}_{t}^{*}
\end{array}\right) & \approx\left(\begin{array}{cc}
\Pi_{1} & \Pi_{2} \\
\Pi_{2} & \Pi_{1}
\end{array}\right)\left(\begin{array}{cc}
\delta_{a} & \delta_{a, a^{*}} \\
\delta_{a, a^{*}} & \delta_{a}
\end{array}\right)\left(\begin{array}{cc}
\Pi_{1} & \Pi_{2} \\
\Pi_{2} & \Pi_{1}
\end{array}\right)^{-1}\left(\begin{array}{c}
\widehat{\bar{r}}_{t-1} \\
\widehat{\widehat{r}}_{t-1}^{*}
\end{array}\right)+\gamma\left(\frac{1+\varphi}{\gamma+\varphi}\right)\left(\begin{array}{cc}
\Pi_{1} & \Pi_{2} \\
\Pi_{2} & \Pi_{1}
\end{array}\right)\left(\begin{array}{c}
\widehat{\varepsilon}_{t}^{a} \\
\widehat{\varepsilon}_{t}^{a *}
\end{array}\right), \\
\left(\begin{array}{c}
\widehat{\varepsilon}_{t}^{a} \\
\widehat{\varepsilon}_{t}^{a *}
\end{array}\right) & \sim N\left(\left(\begin{array}{c}
0 \\
0
\end{array}\right),\left(\begin{array}{cc}
\sigma_{a}^{2} & \rho_{a, a^{*}} \sigma_{a}^{2} \\
\rho_{a, a^{*}} \sigma_{a}^{2} & \sigma_{a}^{2}
\end{array}\right)\right) .
\end{aligned}
$$

where,

$$
\begin{aligned}
\left(\begin{array}{ll}
\Pi_{1} & \Pi_{2} \\
\Pi_{2} & \Pi_{1}
\end{array}\right)^{-1} & =\frac{1}{\left(\Pi_{1}\right)^{2}-\left(\Pi_{2}\right)^{2}}\left(\begin{array}{cc}
\Pi_{1} & -\Pi_{2} \\
-\Pi_{2} & \Pi_{1}
\end{array}\right), \\
\left(\Pi_{1}\right)^{2}-\left(\Pi_{2}\right)^{2} & =\left(\frac{(\gamma+\varphi)(2 \xi-1)}{\varphi\left(\sigma \gamma-(\sigma \gamma-1)(2 \xi-1)^{2}\right)+\gamma}\right)\left(\left(\delta_{a}-1\right)^{2}-\left(\delta_{a, a^{*}}\right)^{2}\right) .
\end{aligned}
$$

Hence, it follows from here that,

$$
\begin{aligned}
& \left(\begin{array}{ll}
\Pi_{1} & \Pi_{2} \\
\Pi_{2} & \Pi_{1}
\end{array}\right)\left(\begin{array}{cc}
\delta_{a} & \delta_{a, a^{*}} \\
\delta_{a, a^{*}} & \delta_{a}
\end{array}\right)\left(\begin{array}{cc}
\Pi_{1} & \Pi_{2} \\
\Pi_{2} & \Pi_{1}
\end{array}\right) \\
& =\frac{1}{\left(\Pi_{1}\right)^{2}-\left(\Pi_{2}\right)^{2}}\left(\begin{array}{ll}
\Pi_{1} & \Pi_{2} \\
\Pi_{2} & \Pi_{1}
\end{array}\right)\left(\begin{array}{cc}
\delta_{a} & \delta_{a, a^{*}} \\
\delta_{a, a^{*}} & \delta_{a}
\end{array}\right)\left(\begin{array}{cc}
\Pi_{1} & -\Pi_{2} \\
-\Pi_{2} & \Pi_{1}
\end{array}\right) \\
& =\left(\begin{array}{cc}
\delta_{a} & \delta_{a, a^{*}} \\
\delta_{a, a^{*}} & \delta_{a}
\end{array}\right),
\end{aligned}
$$

which implies that the structure of the $V A R(1)$ for the natural rates inherits the persistence structure of the underlying productivity shocks. Moreover, we can simplify the notation by expressing the innovations to the natural interest rate process in the following terms,

$$
\begin{aligned}
& \left(\begin{array}{c}
\widehat{\varepsilon}_{t}^{r} \\
\widehat{\varepsilon}_{t}^{r *}
\end{array}\right) \equiv \gamma\left(\frac{1+\varphi}{\gamma+\varphi}\right)\left(\begin{array}{cc}
\Pi_{1} & \Pi_{2} \\
\Pi_{2} & \Pi_{1}
\end{array}\right)\left(\begin{array}{c}
\widehat{\varepsilon}_{t}^{a} \\
\widehat{\varepsilon}_{t}^{a *}
\end{array}\right), \\
& \left(\begin{array}{c}
\widehat{\varepsilon}_{t}^{r} \\
\widehat{\varepsilon}_{t}^{r *}
\end{array}\right) \sim N\left(\left(\begin{array}{c}
0 \\
0
\end{array}\right), \gamma^{2}\left(\frac{1+\varphi}{\gamma+\varphi}\right)^{2}\left(\begin{array}{cc}
\Pi_{1} & \Pi_{2} \\
\Pi_{2} & \Pi_{1}
\end{array}\right)\left(\begin{array}{cc}
\sigma_{a}^{2} & \rho_{a, a^{*}} \sigma_{a}^{2} \\
\rho_{a, a^{*}} \sigma_{a}^{2} & \sigma_{a}^{2}
\end{array}\right)\left(\begin{array}{cc}
\Pi_{1} & \Pi_{2} \\
\Pi_{2} & \Pi_{1}
\end{array}\right)^{T}\right),
\end{aligned}
$$


where,

$$
\begin{aligned}
& \gamma^{2}\left(\frac{1+\varphi}{\gamma+\varphi}\right)^{2}\left(\begin{array}{ll}
\Pi_{1} & \Pi_{2} \\
\Pi_{2} & \Pi_{1}
\end{array}\right)\left(\begin{array}{cc}
\sigma_{a}^{2} & \rho_{a, a^{*}} \sigma_{a}^{2} \\
\rho_{a, a^{*}} \sigma_{a}^{2} & \sigma_{a}^{2}
\end{array}\right)\left(\begin{array}{cc}
\Pi_{1} & \Pi_{2} \\
\Pi_{2} & \Pi_{1}
\end{array}\right)^{T} \\
& \quad=\gamma^{2}\left(\frac{1+\varphi}{\gamma+\varphi}\right)^{2}\left(\begin{array}{ll}
\Pi_{1} & \Pi_{2} \\
\Pi_{2} & \Pi_{1}
\end{array}\right)\left(\begin{array}{cc}
\sigma_{a}^{2} & \rho_{a, a^{*}} \sigma_{a}^{2} \\
\rho_{a, a^{*}} \sigma_{a}^{2} & \sigma_{a}^{2}
\end{array}\right)\left(\begin{array}{cc}
\Pi_{1} & \Pi_{2} \\
\Pi_{2} & \Pi_{1}
\end{array}\right) \\
& \quad=\sigma_{a}^{2} \gamma^{2}\left(\frac{1+\varphi}{\gamma+\varphi}\right)^{2}\left(\begin{array}{cc}
\left(\Pi_{1}\right)^{2}+2 \rho_{a, a^{*}} \Pi_{1} \Pi_{2}+\left(\Pi_{2}\right)^{2} & \rho_{a, a^{*}}\left(\Pi_{1}\right)^{2}+2 \Pi_{1} \Pi_{2}+\rho_{a, a^{*}}\left(\Pi_{2}\right)^{2} \\
\rho_{a, a^{*}}\left(\Pi_{1}\right)^{2}+2 \Pi_{1} \Pi_{2}+\rho_{a, a^{*}}\left(\Pi_{2}\right)^{2} & \left(\Pi_{1}\right)^{2}+2 \rho_{a, a^{*}} \Pi_{1} \Pi_{2}+\left(\Pi_{2}\right)^{2}
\end{array}\right) \\
& \quad=\sigma_{a}^{2} \gamma^{2}\left(\frac{1+\varphi}{\gamma+\varphi}\right)^{2}\left(\left(\Pi_{1}\right)^{2}+2 \rho_{a, a^{*}} \Pi_{1} \Pi_{2}+\left(\Pi_{2}\right)^{2}\right)\left(\begin{array}{cc}
1 & \frac{\rho_{a, a^{*}}\left(\Pi_{1}\right)^{2}+2 \Pi_{1} \Pi_{2}+\rho_{a, a^{*}}\left(\Pi_{2}\right)^{2}}{\left(\Pi_{1}\right)^{2}+2 \rho_{a, a^{*}} \Pi_{1} \Pi_{2}+\left(\Pi_{2}\right)^{2}} \\
\frac{\rho_{a, a^{*}}\left(\Pi_{1}\right)^{2}+2 \Pi_{1} \Pi_{2}+\rho_{a, a^{*}}\left(\Pi_{2}\right)^{2}}{\left(\Pi_{1}\right)^{2}+2 \rho_{a, a^{*}} \Pi_{1} \Pi_{2}+\left(\Pi_{2}\right)^{2}} & 1
\end{array}\right) .
\end{aligned}
$$

Hence, we define the volatility and the correlation of the natural rate innovations in the following fashion,

$$
\begin{aligned}
\sigma_{r}^{2} & =\sigma_{a}^{2} \gamma^{2}\left(\frac{1+\varphi}{\gamma+\varphi}\right)^{2}\left(\left(\Pi_{1}\right)^{2}+2 \rho_{a, a^{*}} \Pi_{1} \Pi_{2}+\left(\Pi_{2}\right)^{2}\right), \\
\rho_{r, r^{*}} & =\frac{\rho_{a, a^{*}}\left(\Pi_{1}\right)^{2}+2 \Pi_{1} \Pi_{2}+\rho_{a, a^{*}}\left(\Pi_{2}\right)^{2}}{\left(\Pi_{1}\right)^{2}+2 \rho_{a, a^{*}} \Pi_{1} \Pi_{2}+\left(\Pi_{2}\right)^{2}} .
\end{aligned}
$$


C Tables and Figures 


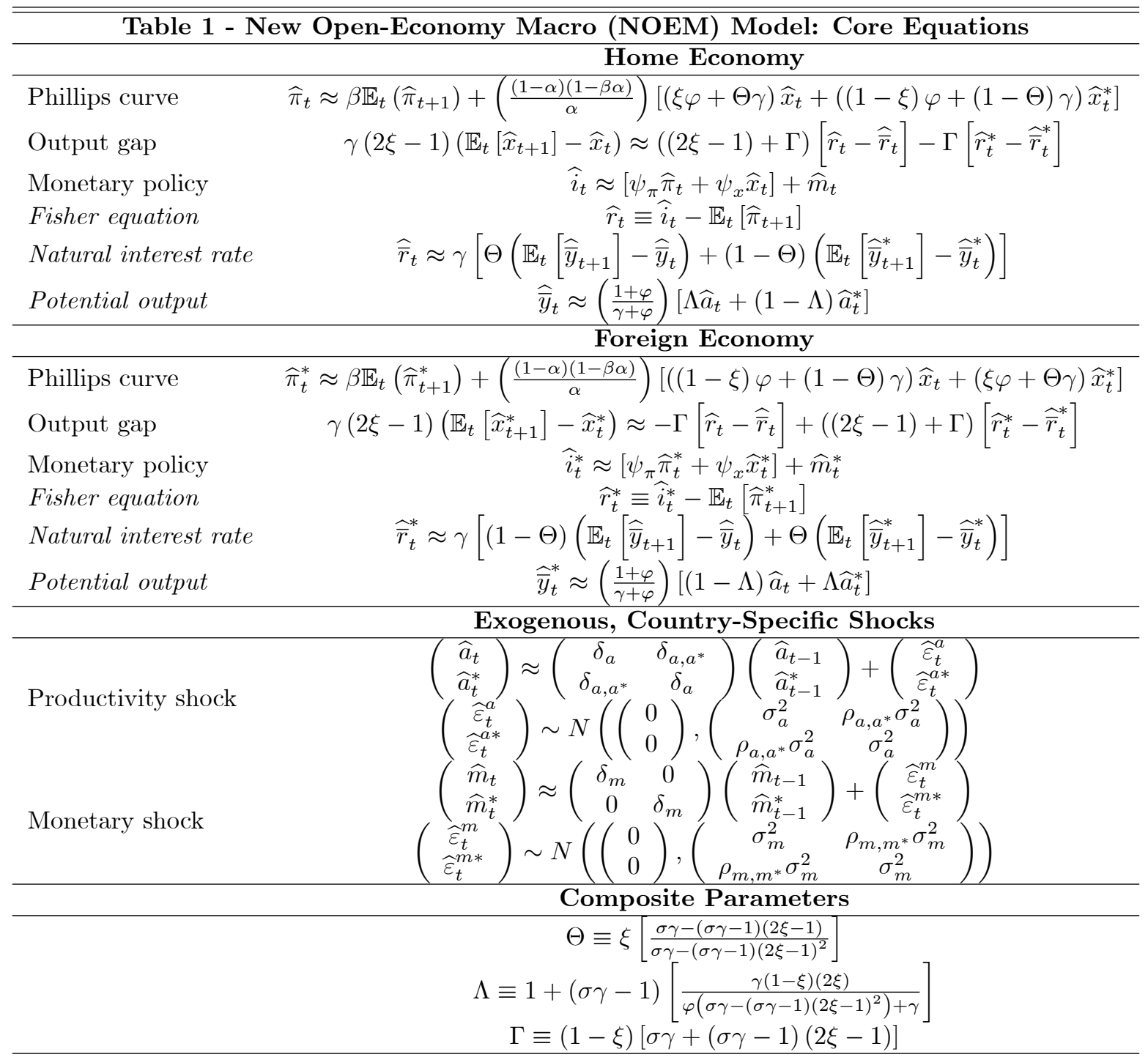




\begin{tabular}{|c|c|}
\hline Table 2 - New Open-1 & $\begin{array}{c}\text { omy Macro (NOEM) Model: Non-Core Equatio } \\
\text { Home Economy }\end{array}$ \\
\hline Output & $\widehat{y}_{t}=\widehat{\bar{y}}_{t}+\widehat{x}_{t}$ \\
\hline Consumption & $\widehat{c}_{t} \approx \Theta \widehat{y}_{t}+(1-\Theta) \widehat{y}_{t}^{*}$ \\
\hline Employment & $\widehat{l}_{t} \approx \widehat{y}_{t}-\widehat{a}_{t}$ \\
\hline \multirow[t]{2}{*}{ Real wages } & $\left(\widehat{w}_{t}-\widehat{p}_{t}\right) \approx \gamma \widehat{c}_{t}+\varphi \widehat{l}_{t} \approx(\varphi+\gamma \Theta) \widehat{y}_{t}+\gamma(1-\Theta) \widehat{y}_{t}^{*}-\varphi \widehat{a}_{t}$ \\
\hline & Foreign Economy \\
\hline Output & $\widehat{y}_{t}^{*}=\widehat{\bar{y}}_{t}^{*}+\widehat{x}_{t}^{*}$ \\
\hline Consumption & $\widehat{c}_{t}^{*} \approx(1-\Theta) \widehat{y}_{t}+\Theta \widehat{y}_{t}^{*}$ \\
\hline Employment & $\widehat{l}_{t}^{*} \approx \widehat{y}_{t}^{*}-\widehat{a}_{t}^{*}$ \\
\hline \multirow[t]{2}{*}{ Real wages } & $\left(\widehat{w}_{t}^{*}-\widehat{p}_{t}^{*}\right) \approx \gamma \widehat{c}_{t}^{*}+\varphi \widehat{l}_{t}^{*} \approx \gamma(1-\Theta) \widehat{y}_{t}+(\varphi+\gamma \Theta) \widehat{y}_{t}^{*}-\varphi \widehat{a}_{t}^{*}$ \\
\hline & International Relative Prices and Trade \\
\hline Real exchange rate & $\widehat{r s}_{t} \approx(2 \xi-1) \widehat{t o t}_{t}$ \\
\hline Terms of trade & $\widehat{\operatorname{tot}}_{t} \approx\left|\frac{\gamma}{\sigma \gamma-(\sigma \gamma-1)(2 \xi-1)^{2}}\right|\left(\widehat{y}_{t}-\widehat{y}_{t}^{*}\right)$ \\
\hline Home real exports & $\overline{\exp }_{t} \approx \Xi \widehat{y}_{t}+(1-\Xi) \widehat{y}_{t}^{*}$ \\
\hline Home real imports & $\widehat{i m p}_{t} \approx-(1-\Xi) \widehat{y}_{t}-\Xi \widehat{y}_{t}^{*}$ \\
\hline \multirow[t]{4}{*}{ Home real trade balance } & $\widehat{t b}_{t} \equiv \widehat{y}_{t}-\widehat{c}_{t}=(1-\xi)\left(\widehat{\exp }_{t}-\widehat{i m p}_{t}\right) \approx(1-\Theta)\left(\widehat{y}_{t}-\widehat{y}_{t}^{*}\right)$ \\
\hline & Composite Parameters \\
\hline & $\Theta \equiv \xi\left[\frac{\sigma \gamma-(\sigma \gamma-1)(2 \xi-1)}{\sigma \gamma-(\sigma \gamma-1)(2 \xi-1)^{2}}\right]$ \\
\hline & $\Xi \equiv\left[\frac{\sigma \gamma+(\sigma \gamma-1)(2 \xi-1)(1-\xi)}{\sigma \gamma-(\sigma \gamma-1)(2 \xi-1)^{2}}\right]$ \\
\hline
\end{tabular}




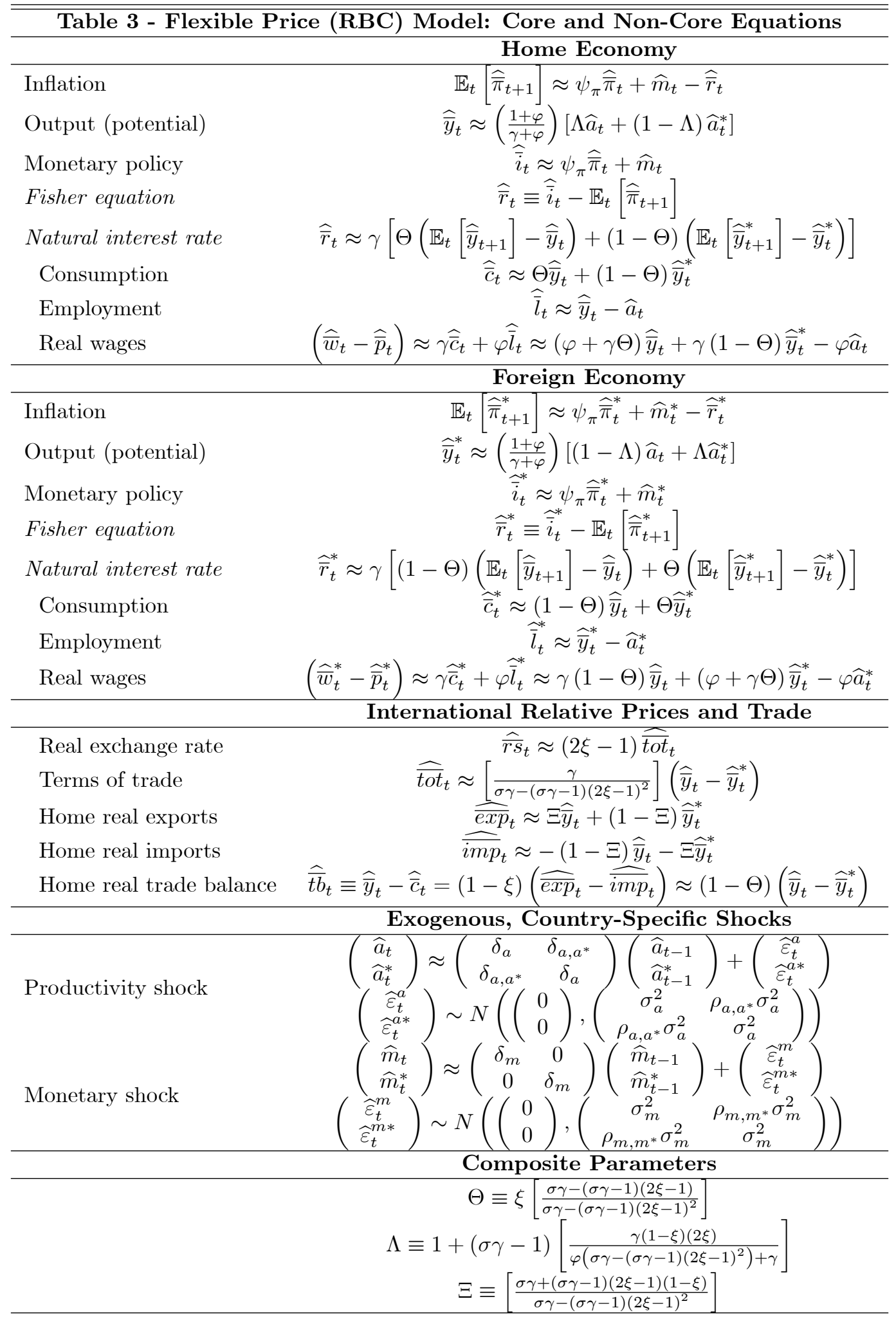




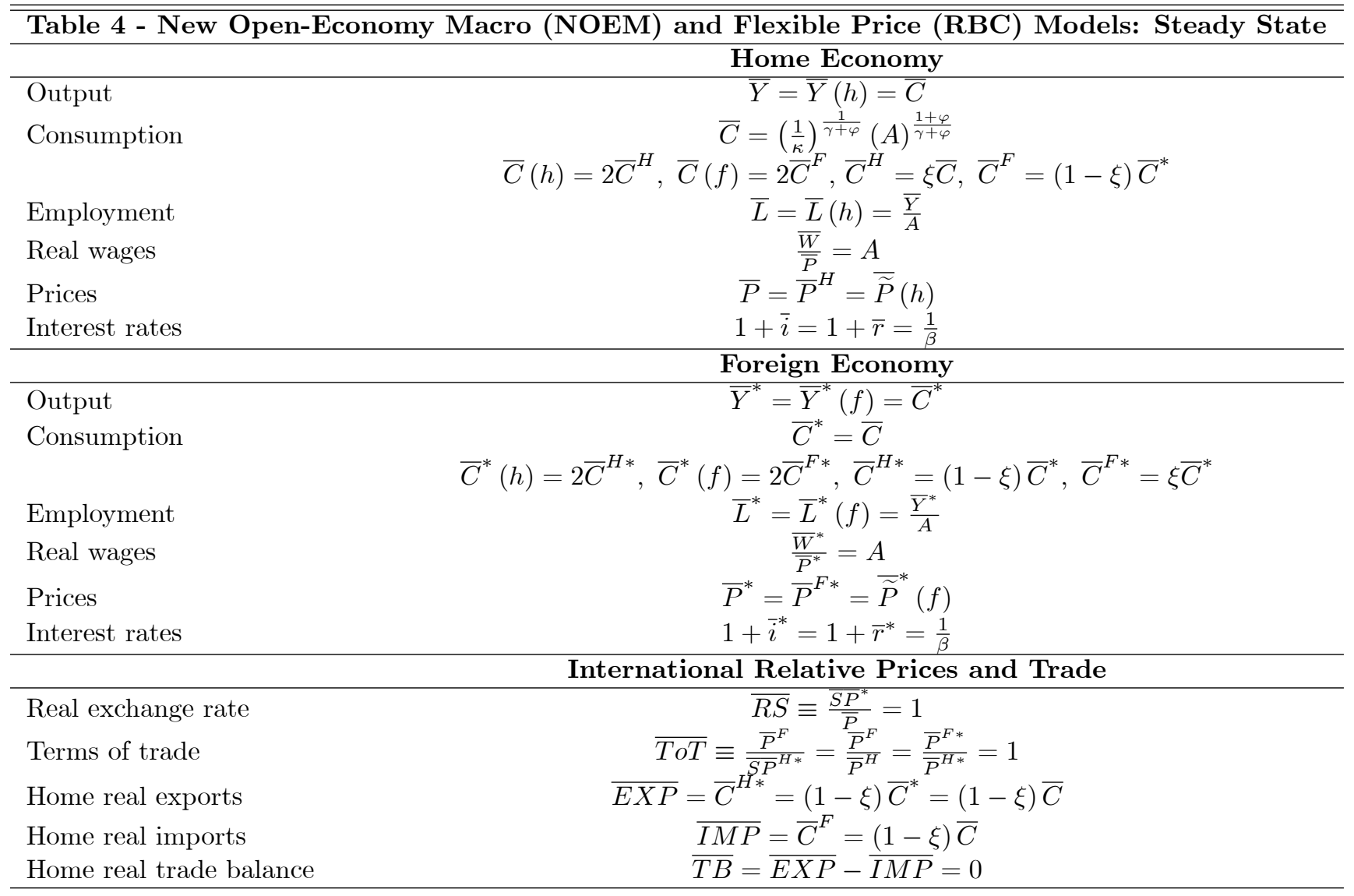




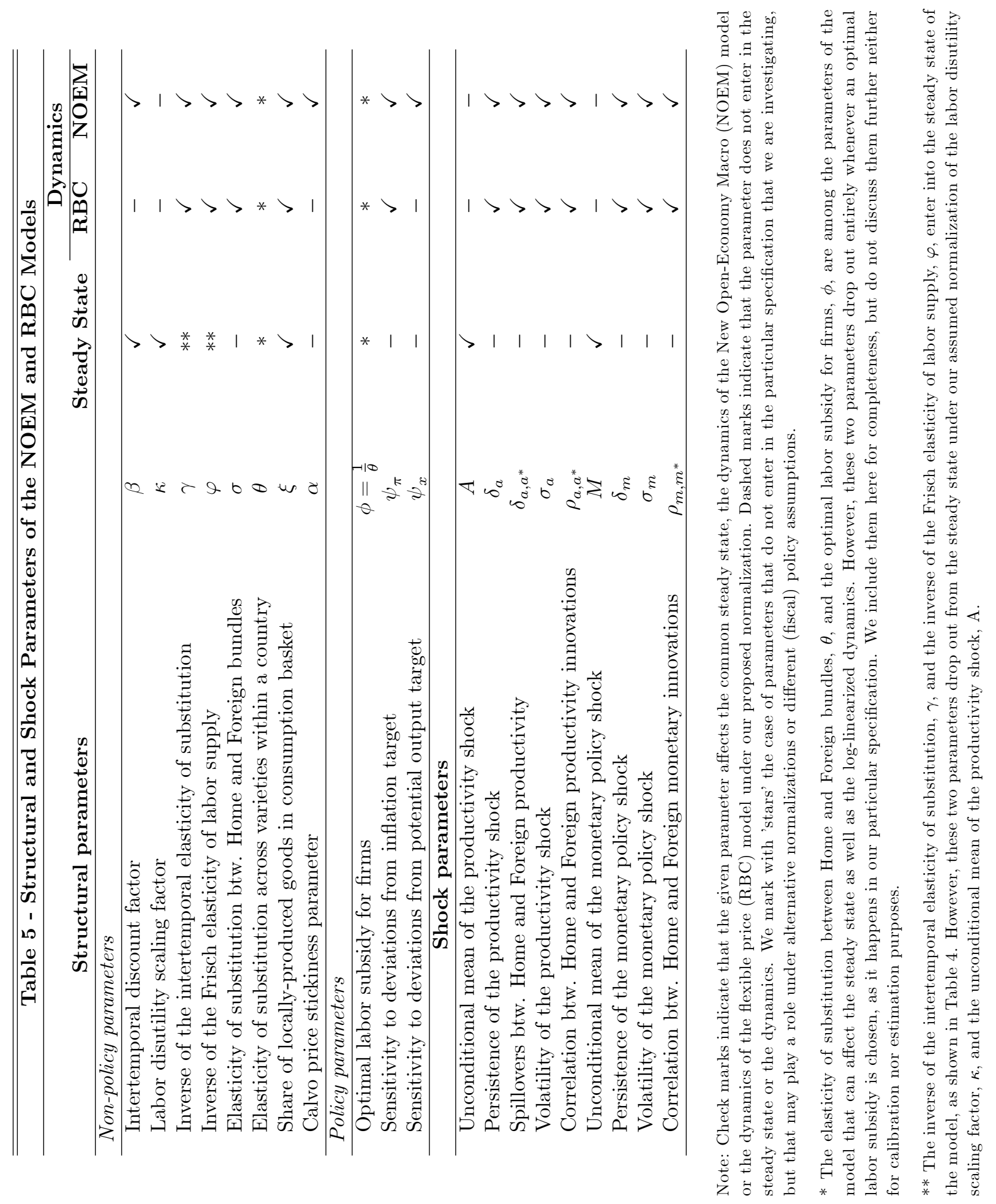




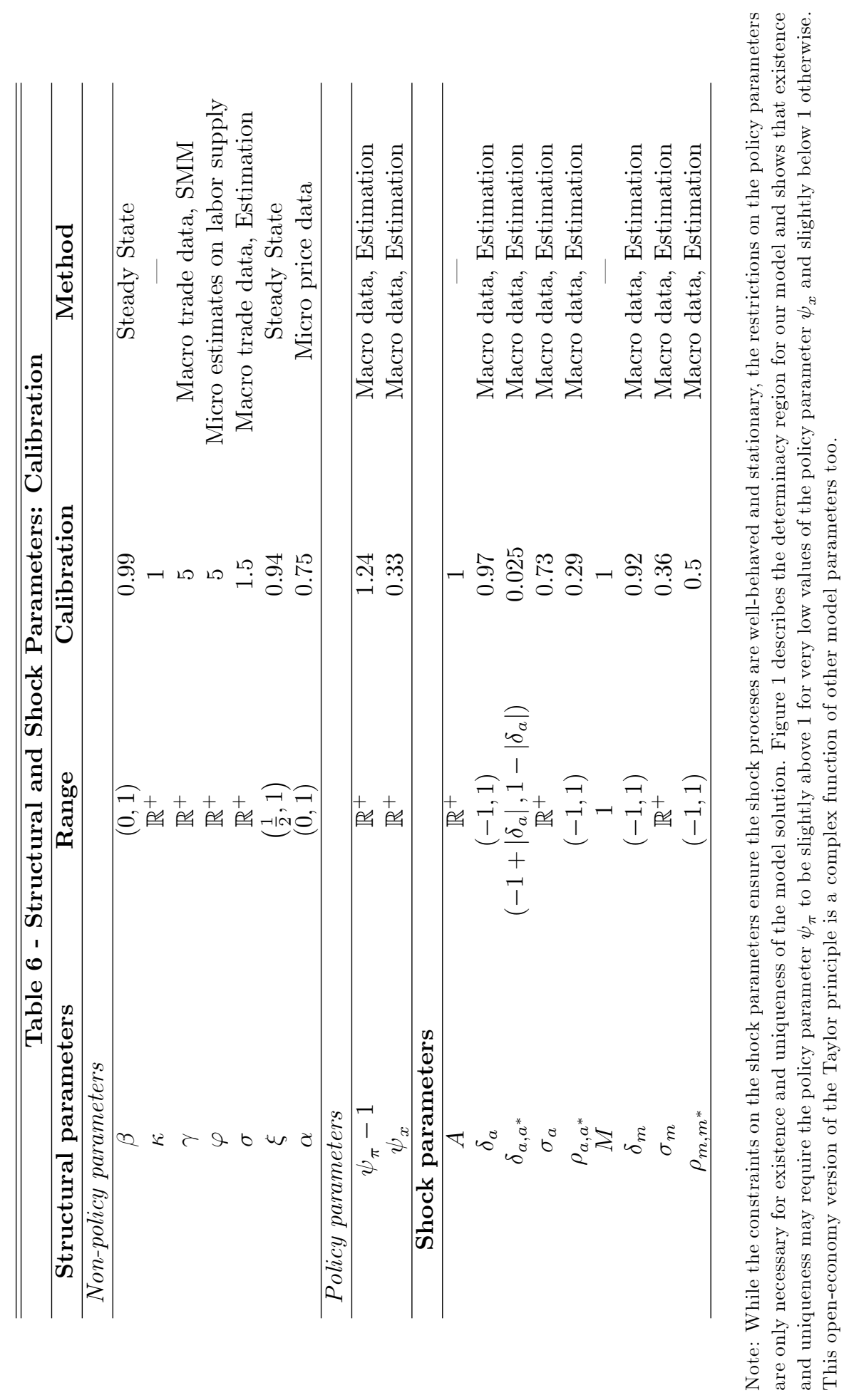




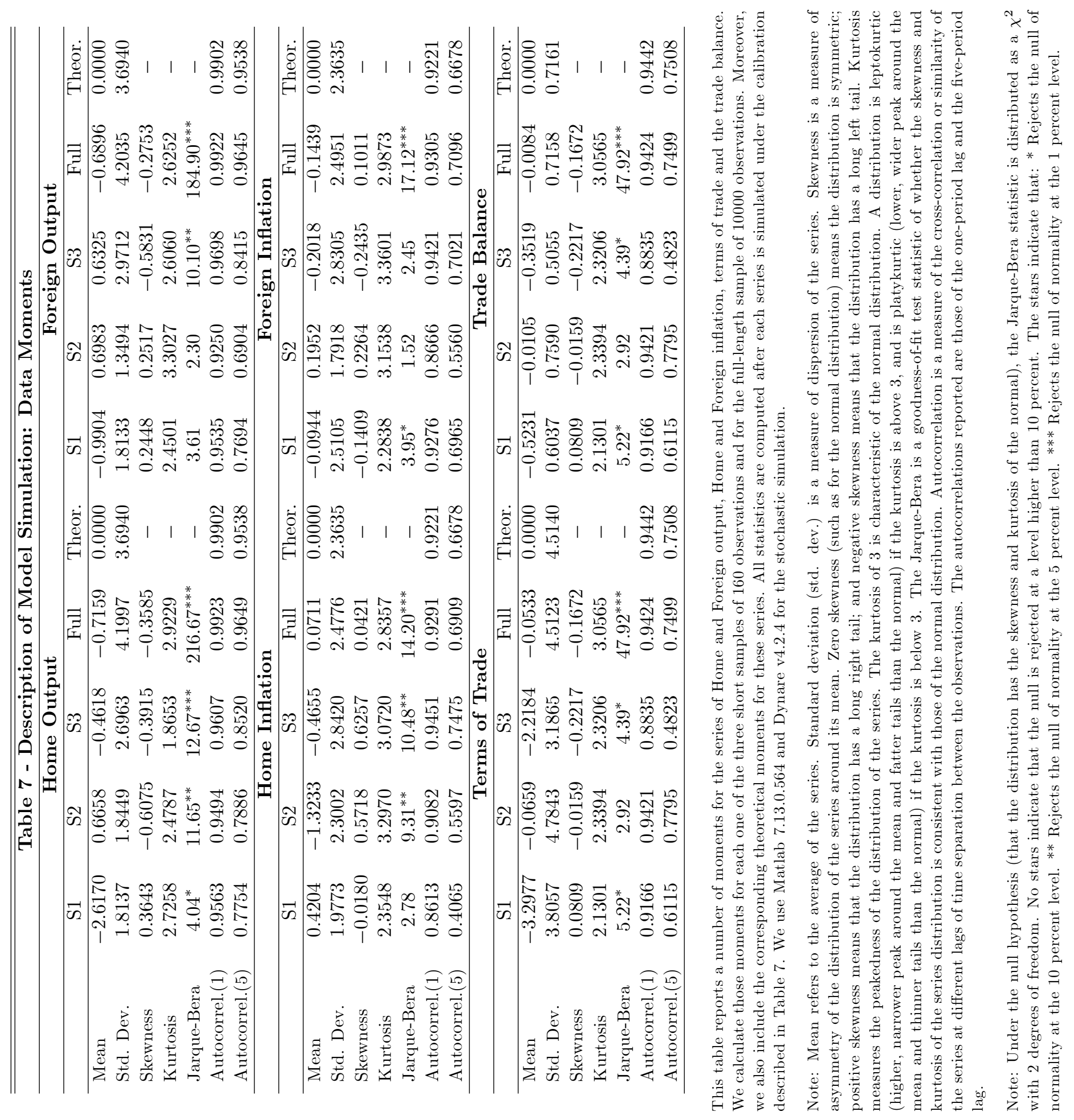




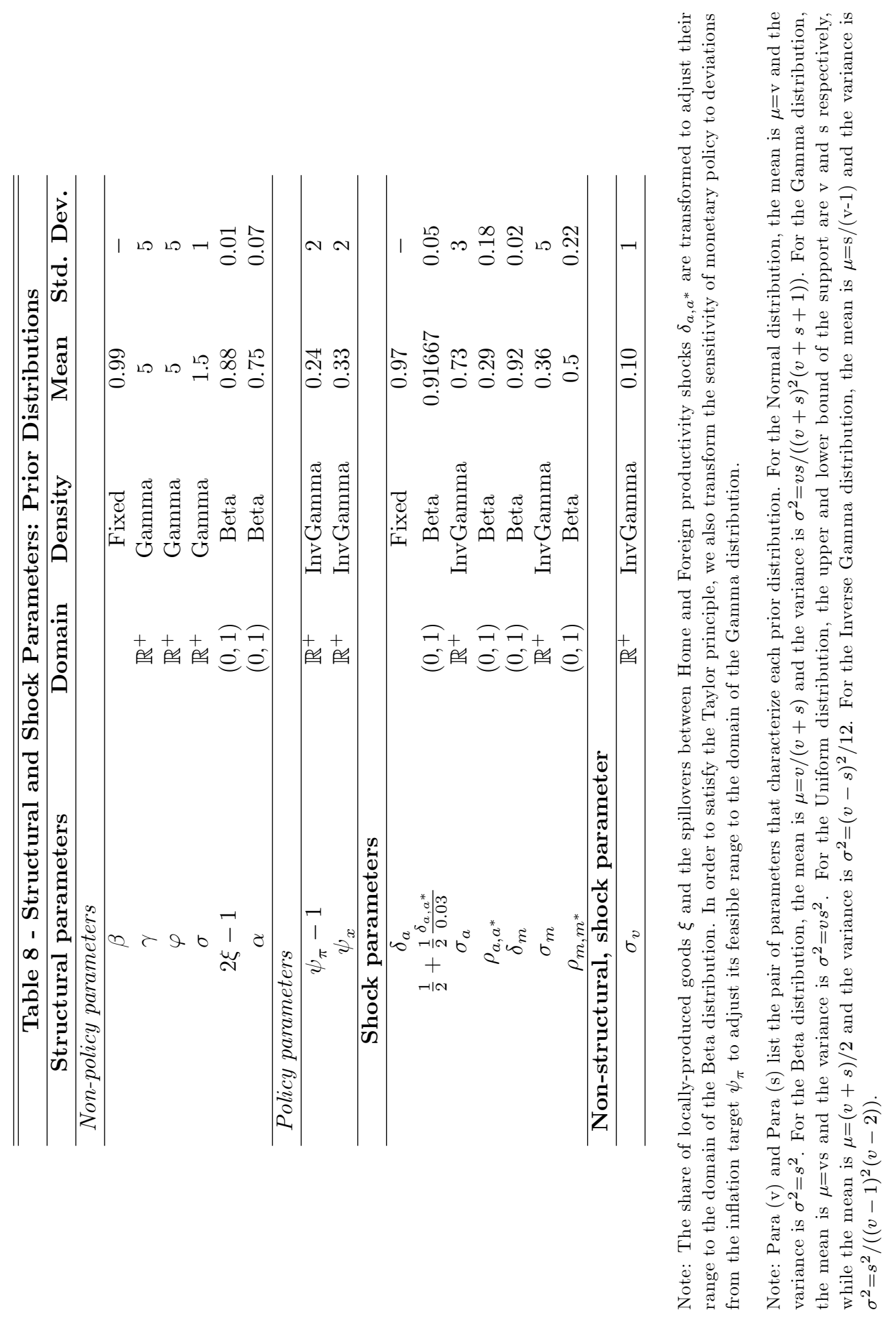




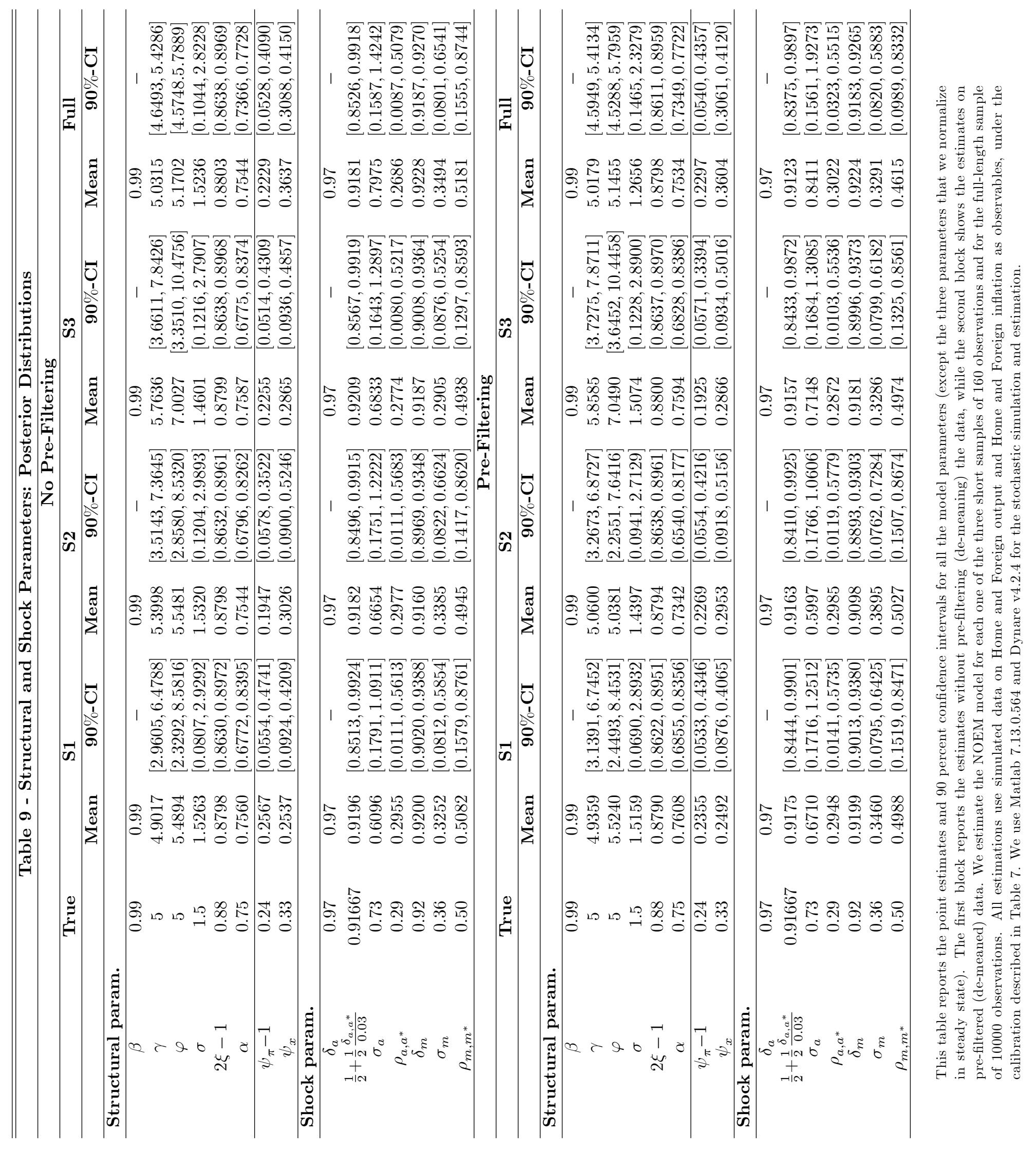




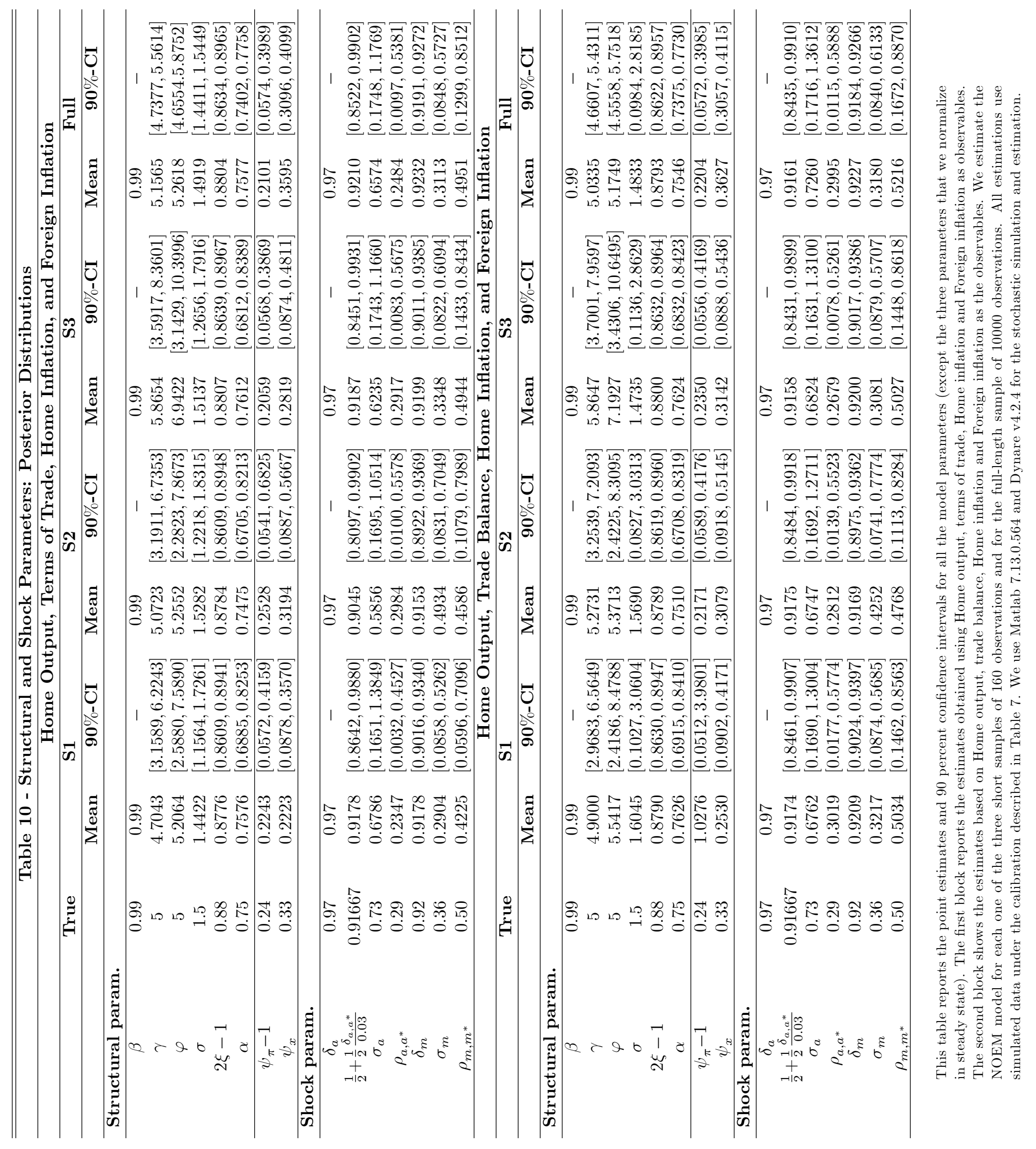




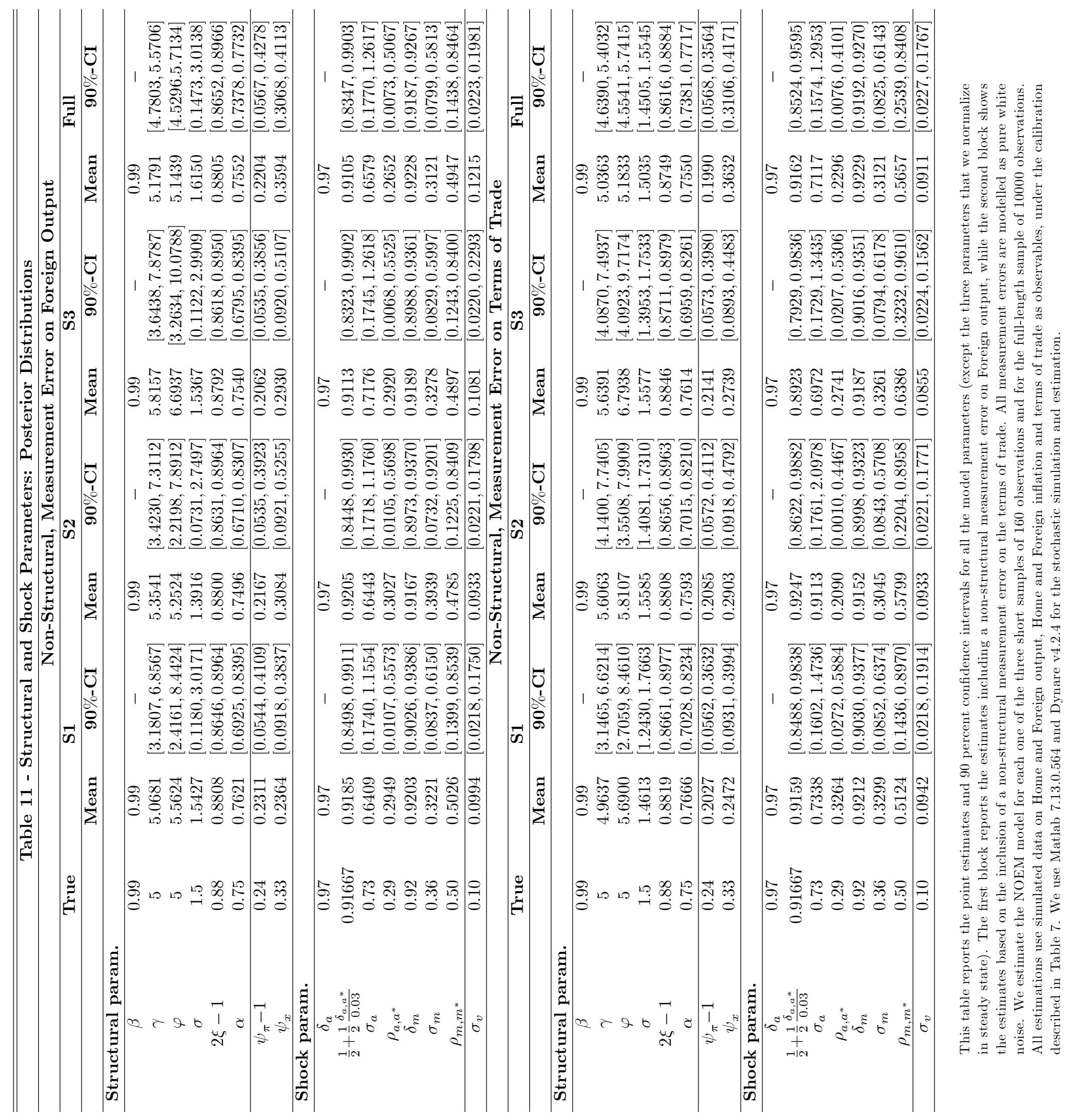




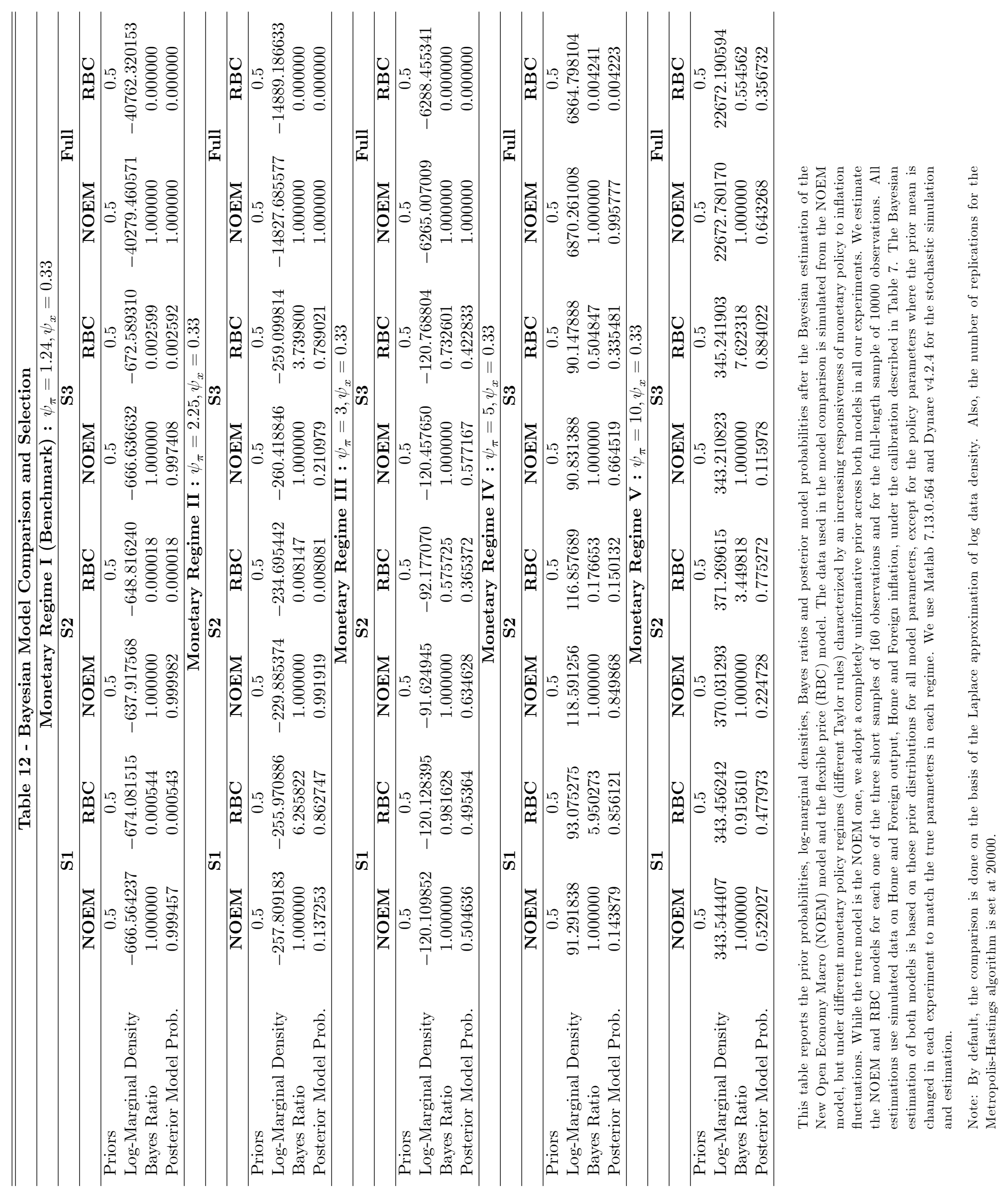


FIGURE 1. Determinacy Region of the Parameter Space (Open-Economy Taylor Principle)

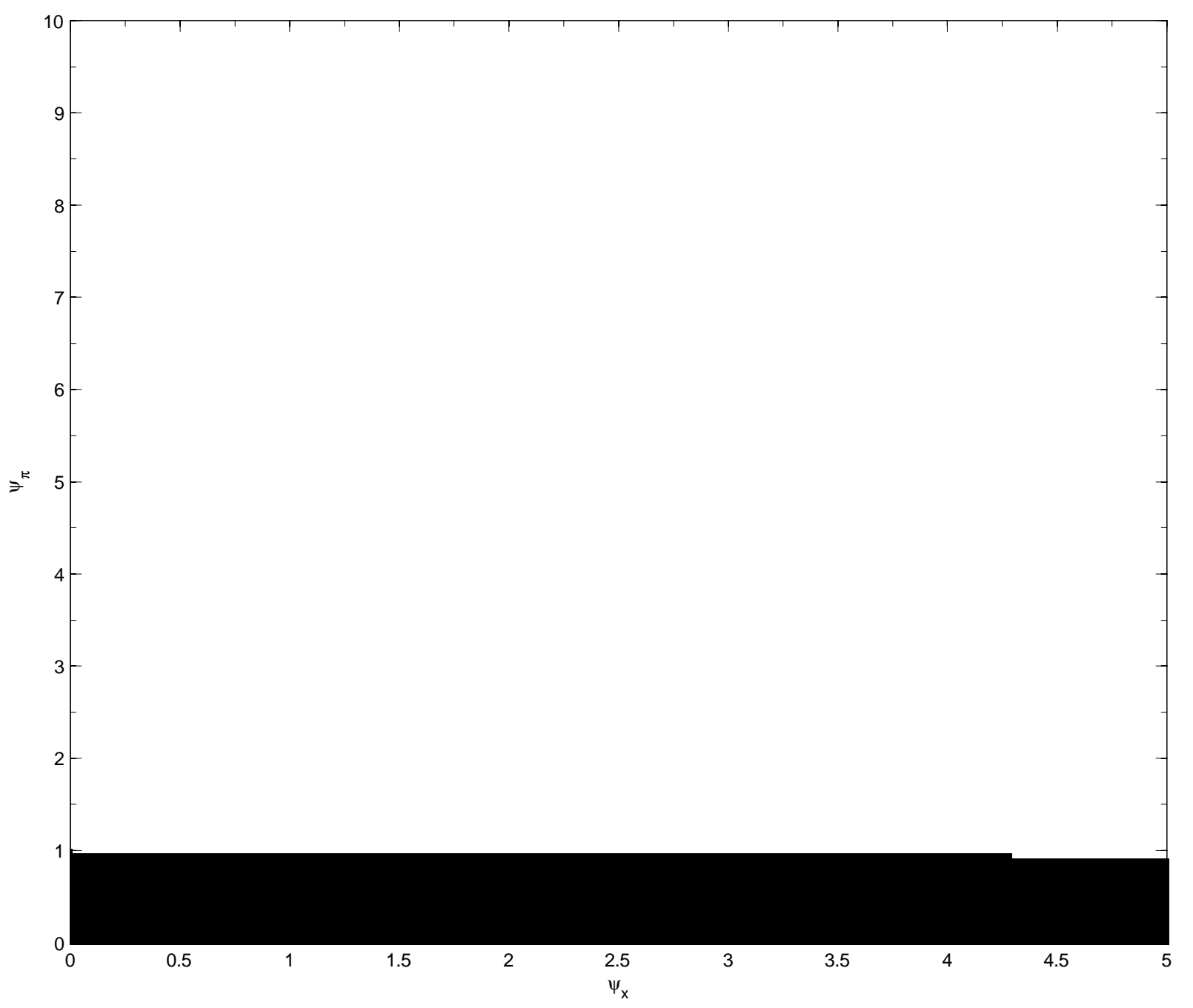

Note: The model is solved for the eigenvalues of its solution with code written for Dynare version 4.2 .2 and Matlab version 7.13.0.564, taking the non-policy structural and exogenous parameters of the NOEM model as given and spanning the space for the policy parameters over a grid. This figure plots the relevant subset of the policy parameter space for our Bayesian investigation of the NOEM model and marks in black the area where the solution is indeterminate (multiple solutions) based on the Blanchard-Kahn conditions and in white where the solution exists and is unique. The code used to describe the determinacy region of the parameter space is available upon request from the authors. 


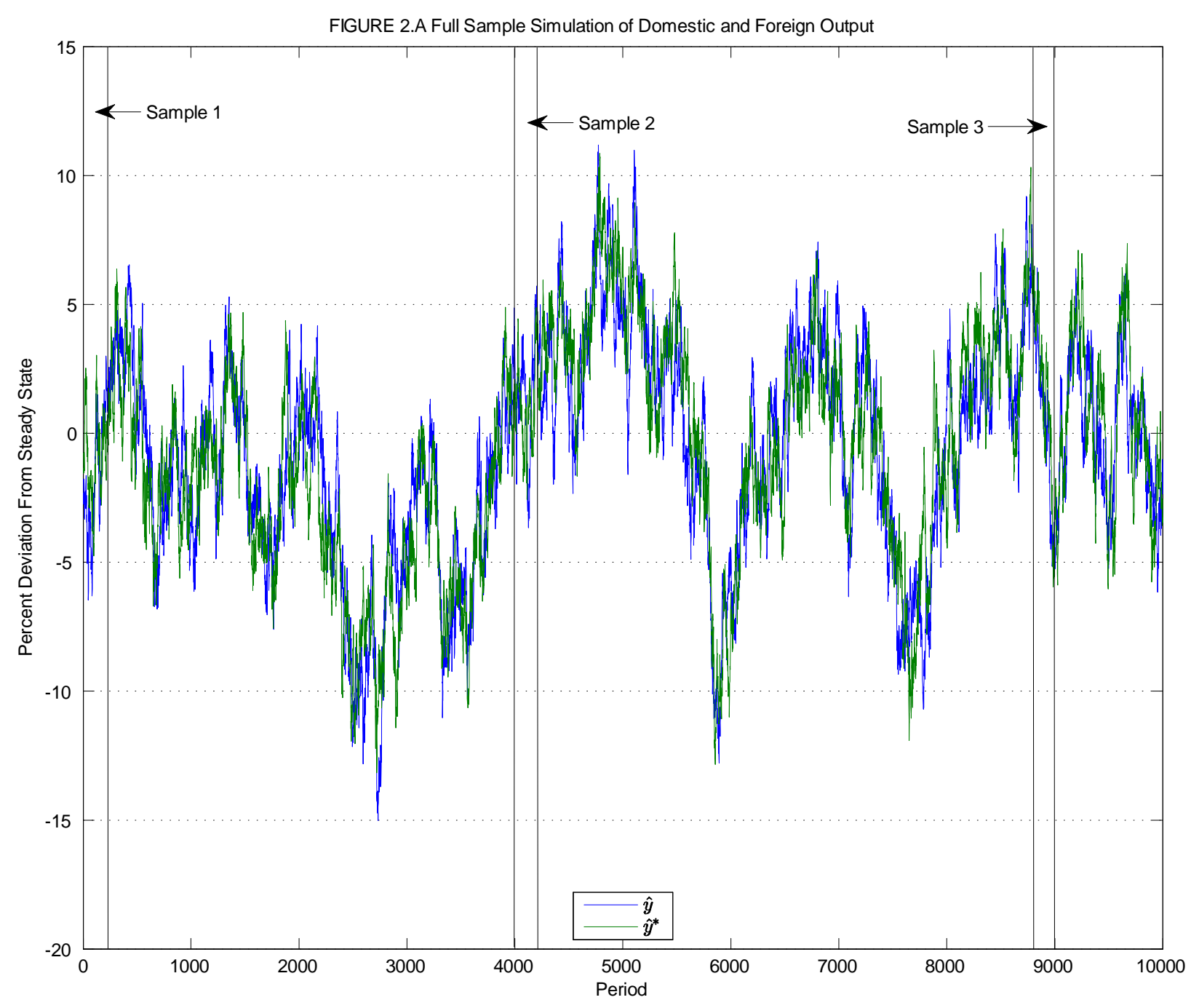

Note: The model is simulated over 10000 periods with code written for Dynare version 4.2.2 and Matlab version 7.13.0.564. This figure plots the entire realization for domestic and foreign output. The code for the simulation is available upon request from the authors. 


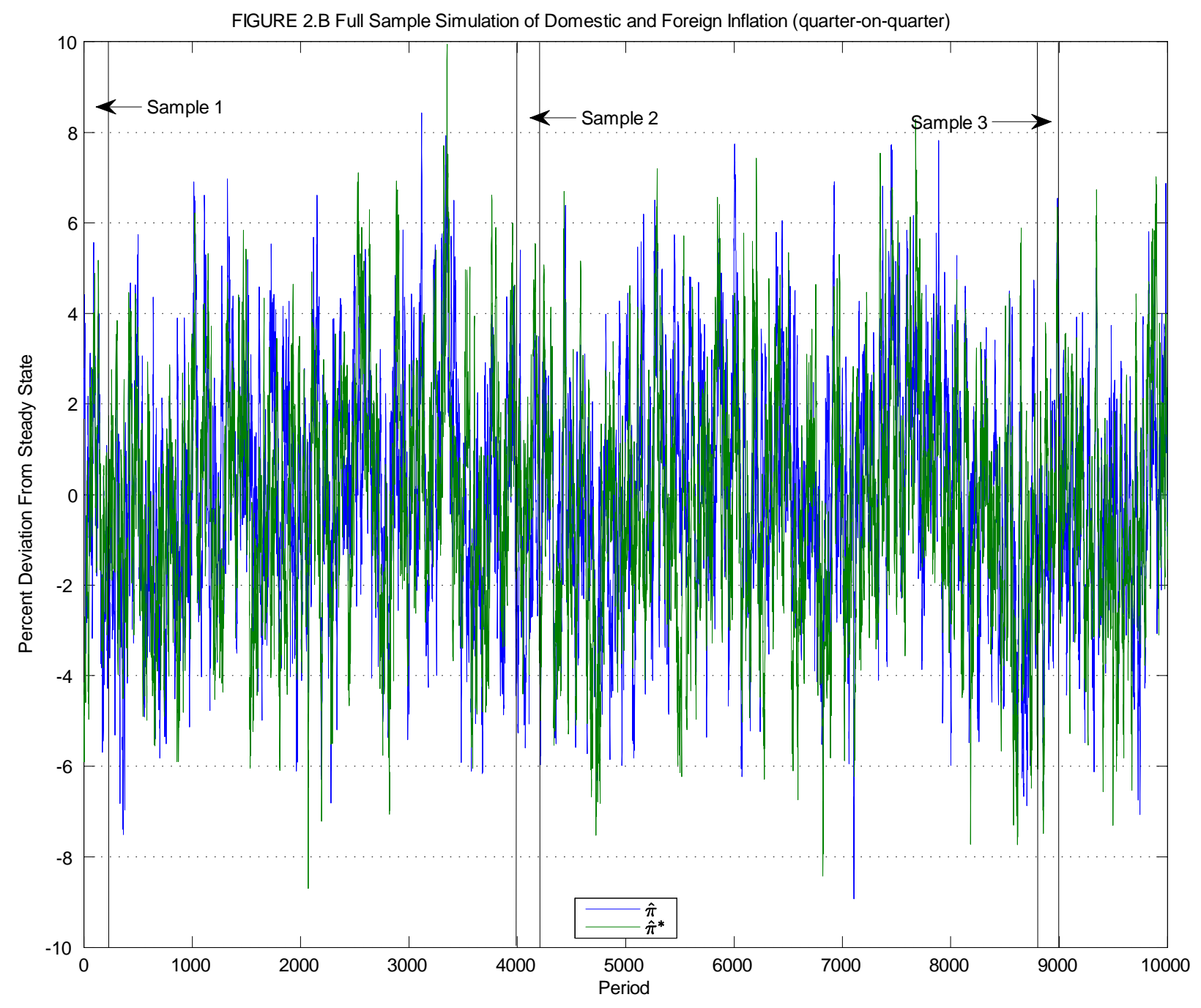

Note: The model is simulated over 10000 periods with code written for Dynare version 4.2.2 and Matlab version 7.13.0.564. This figure plots the entire realization for domestic and foreign inflation. The code for the simulation is available upon request from the authors. 


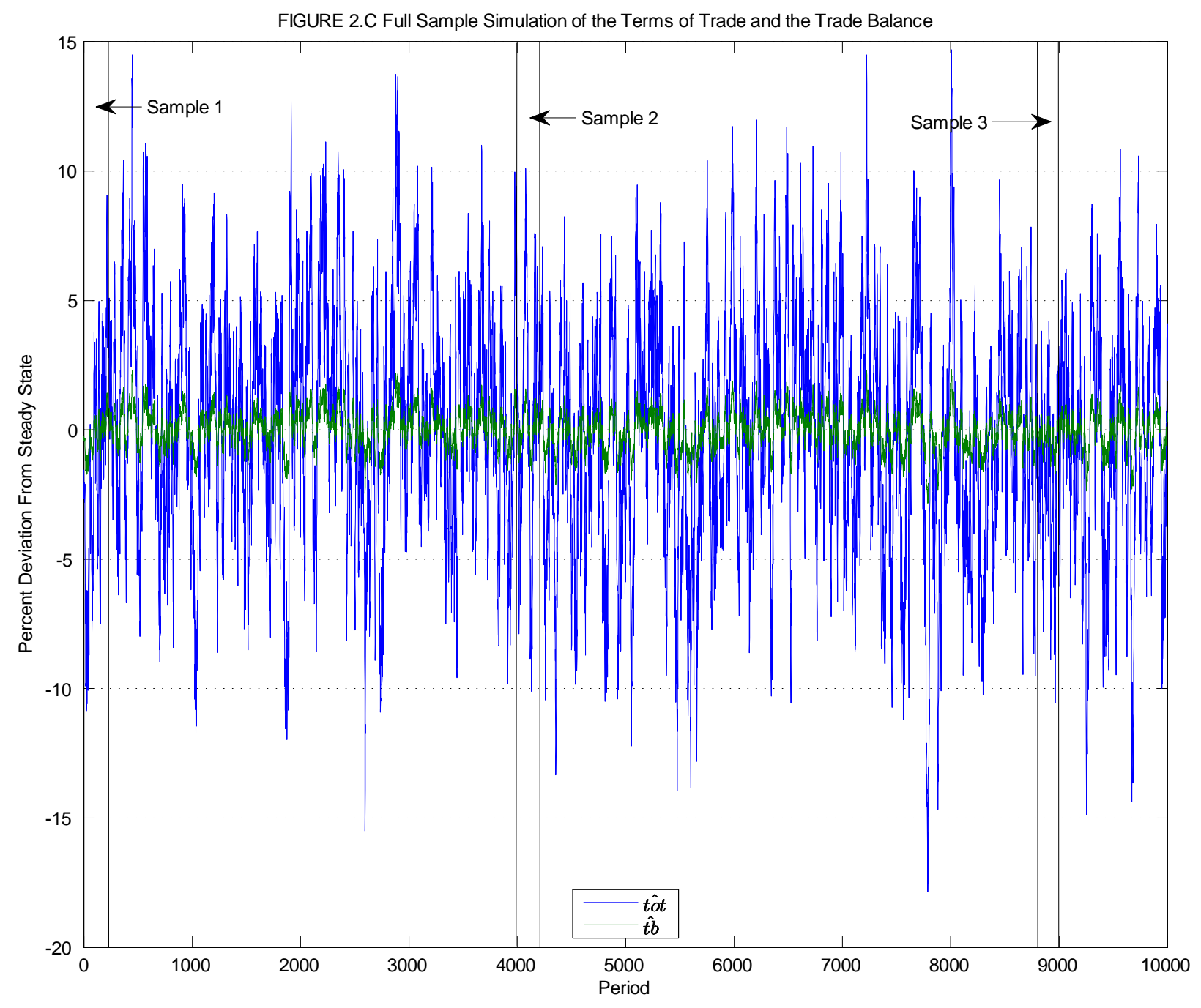

Note: The model is simulated over 10000 periods with code written for Dynare version 4.2.2 and Matlab version 7.13.0.564. This figure plots the entire realization for terms of trade and the trade balance. The code for the simulation is available upon request from the authors. 
FIGURE 3. The Three Sub-Samples Of Simulated Data Used for Bayesian Estimation
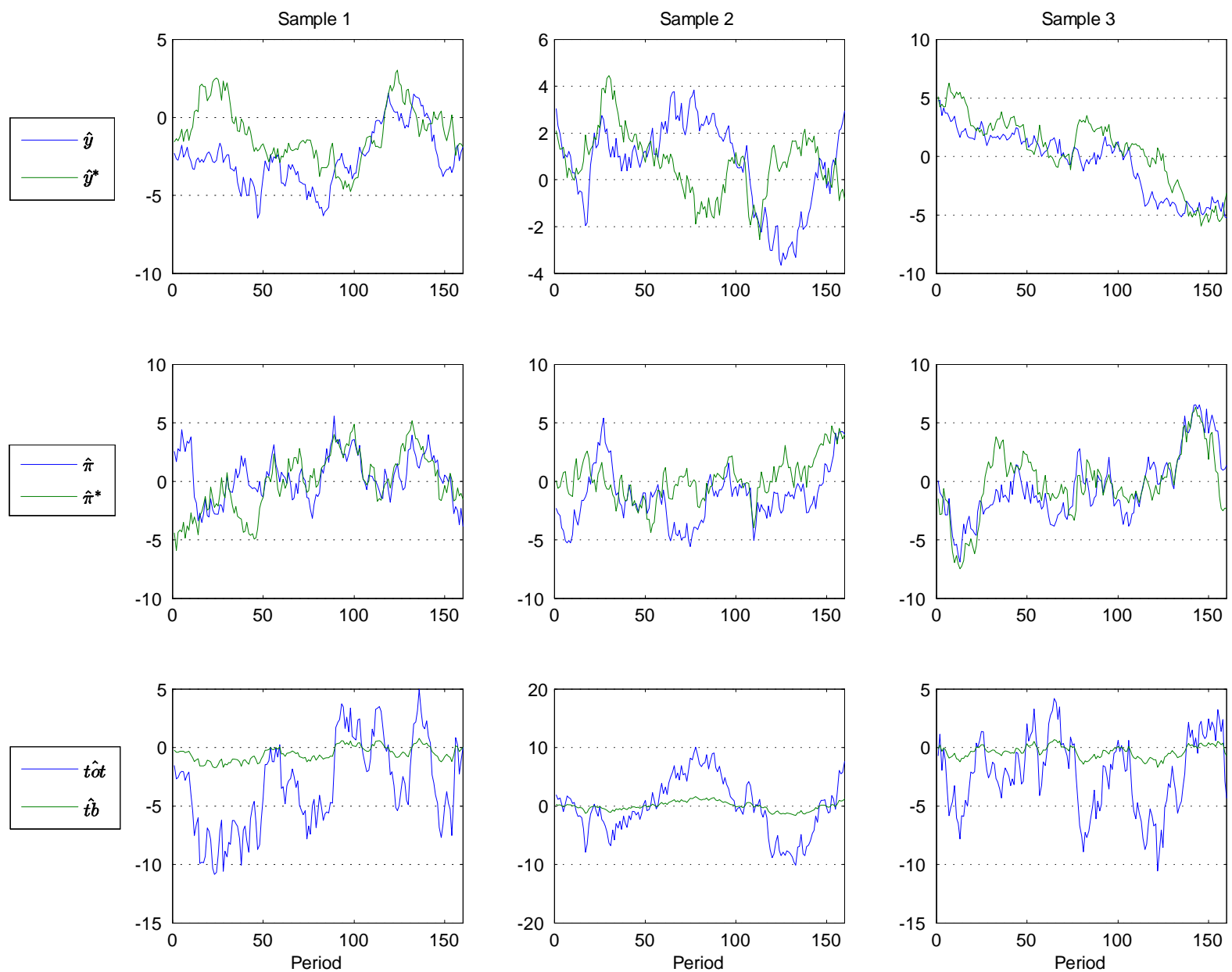

Note: The model is simulated over 10000 periods with code written for Dynare version 4.2.2 and Matlab version 7.13.0.564. We select three sub-samples of 160 periods each for estimation purposes. This figure illustrates the realization of each sub-sample including domestic and foreign output in the top panel, domestic and foreign inflation in the middle panel, and the terms of trade and trade balance in the bottom panel. Each sub-sample is plotted in a different column. The code for the simulation is available upon request from the authors. 
FIGURE 4. Scatter Plots Of Domestic Inflation Against Other Endogenous Variables For The Three Simulated Sub-Samples Used
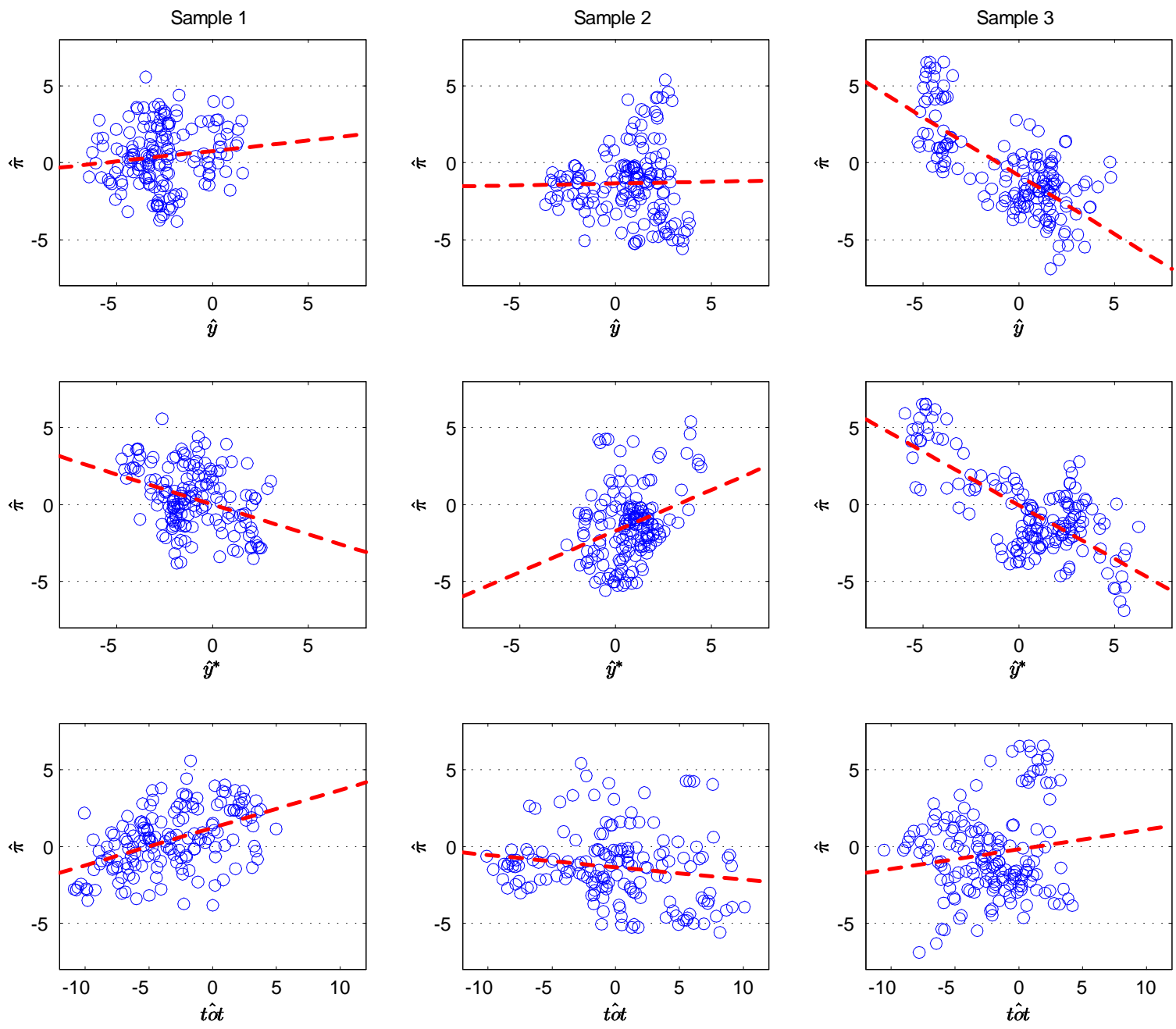

○ Simulated Data - - - Least-Squares Line

Note: The model is simulated over 10000 periods with code written for Dynare version 4.2 .2 and Matlab version 7.13.0.564. We select three sub-samples of 160 periods each for estimation purposes. Each sub-sample is plotted in a different column. This figure illustrates the linear relationship apparent in each sub-sample between domestic inflation and domestic output (top panel), domestic inflation and foreign output (middle panel), and domestic inflation and terms of trade (bottom panel). The dashed red line is the least-squares line that summarizes the type of relationship that we find between the data in each scatter plot. The code for the simulation is available upon request from the authors. 

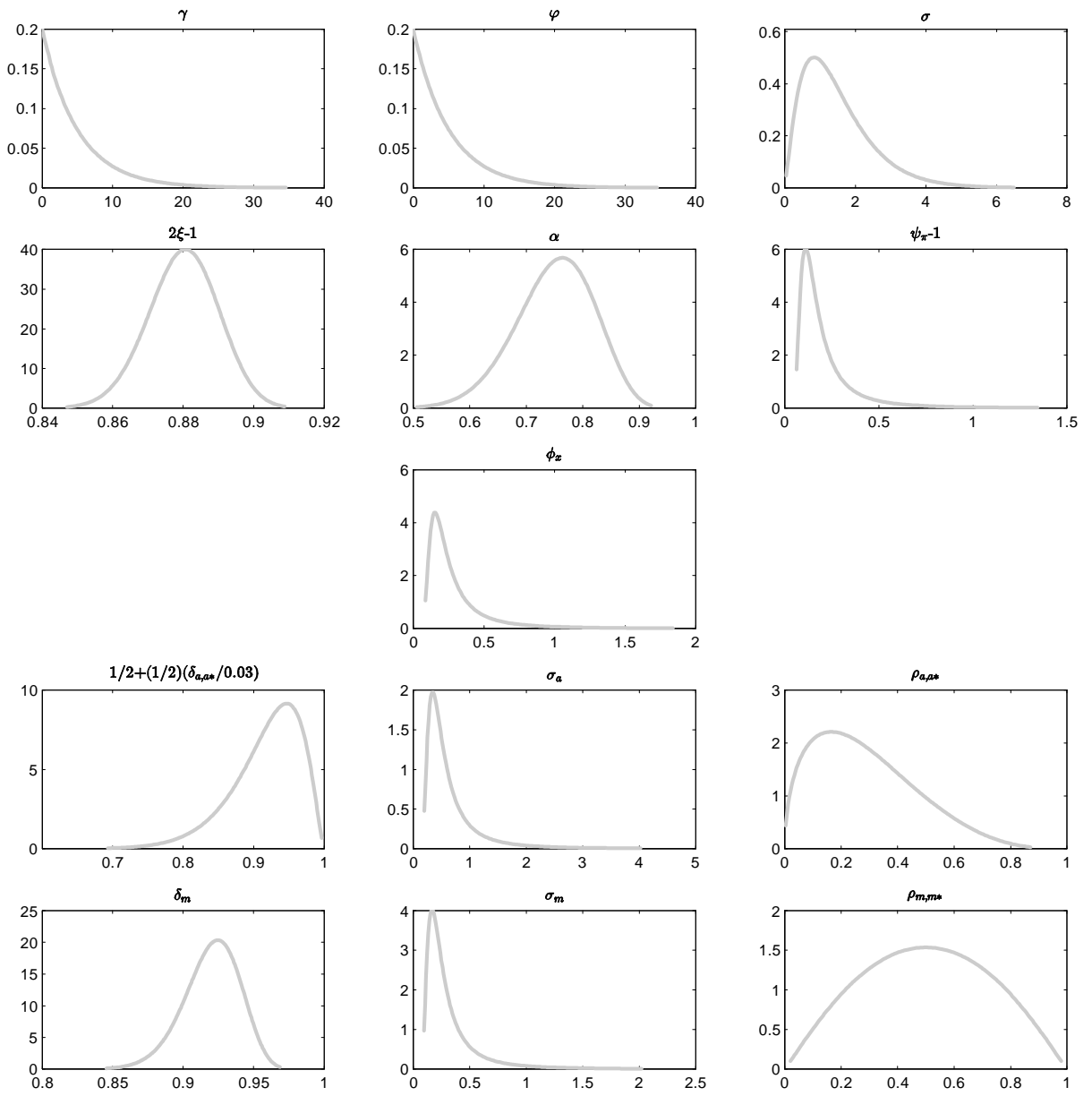

Note: The code is written for Matlab version 7.13.0.564. This figure plots the prior distributions of all 13 structural (policy and non-policy) and shock parameters that do not receive a degenerate prior distribution, The code for this chart is available upon request from the authors. 
FIGURE 6.A Prior and Posterior Distributions for the Structural and Shock Parameters - Short Sample, S1
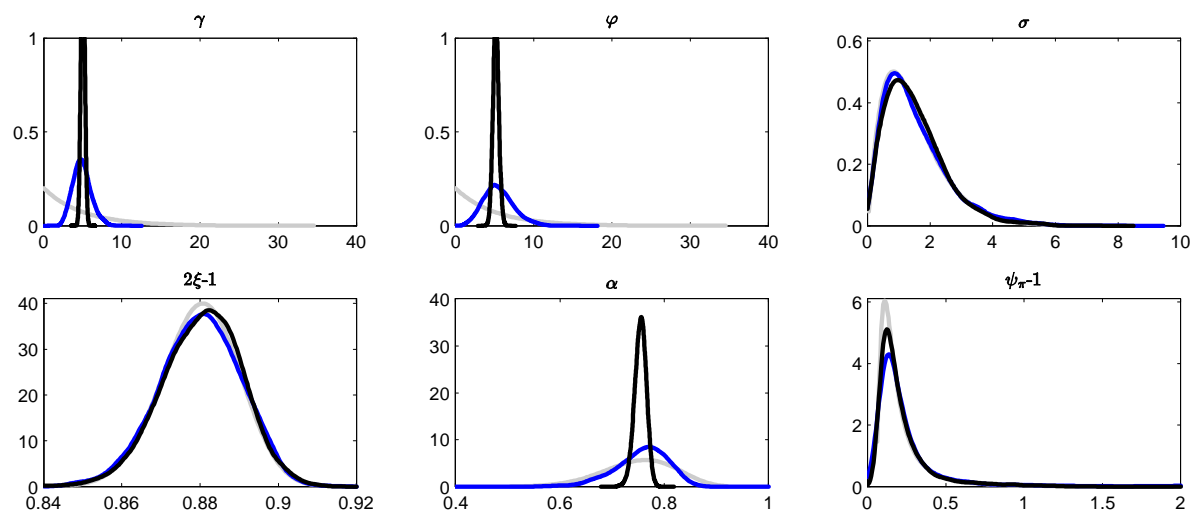

$\phi_{x}$
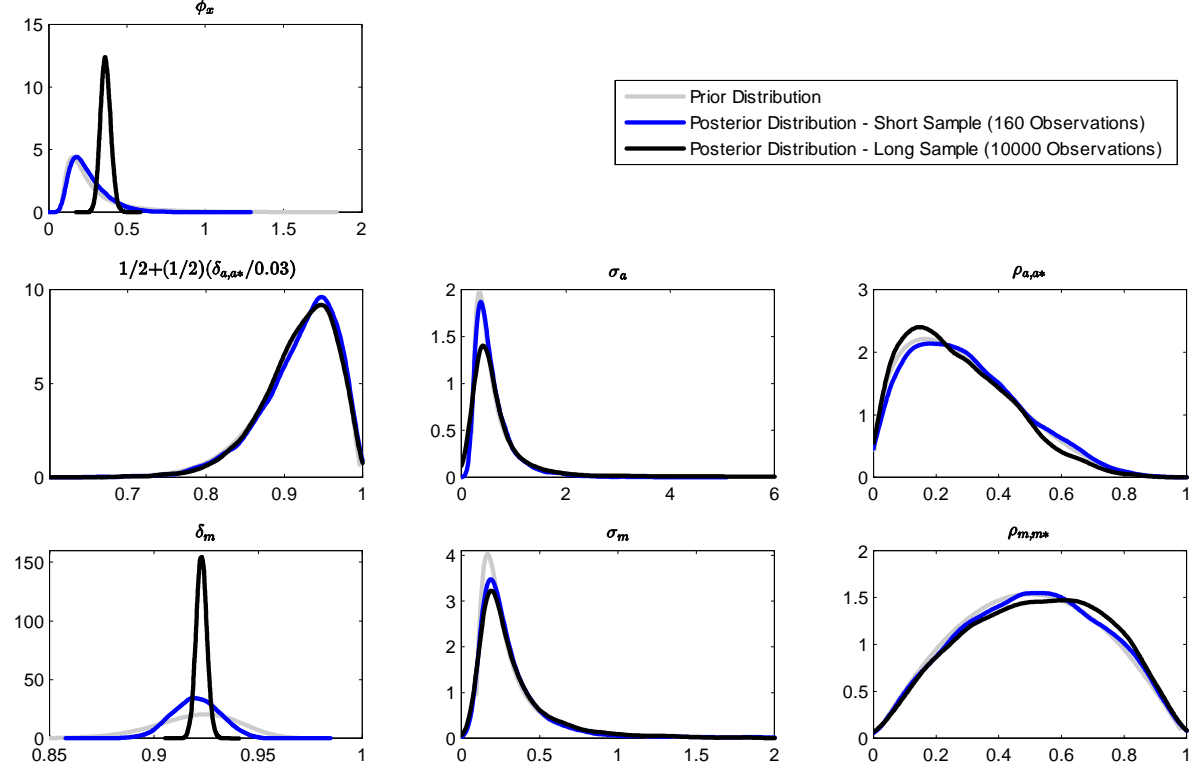

Note: The code is written for Matlab version 7.13.0.564. This figure plots the prior distributions of all 13 structural (policy and non-policy) and shock parameters that do not receive a degenerate prior distribution, It also includes the posterior distribution estimated from a short sample of 160 observations (S1) and the long sample of 10000 observations under the benchmark implementation based on four observables (Home and Foreign output, Home and Foreign inflation). The code for this chart is available upon request from the authors. 

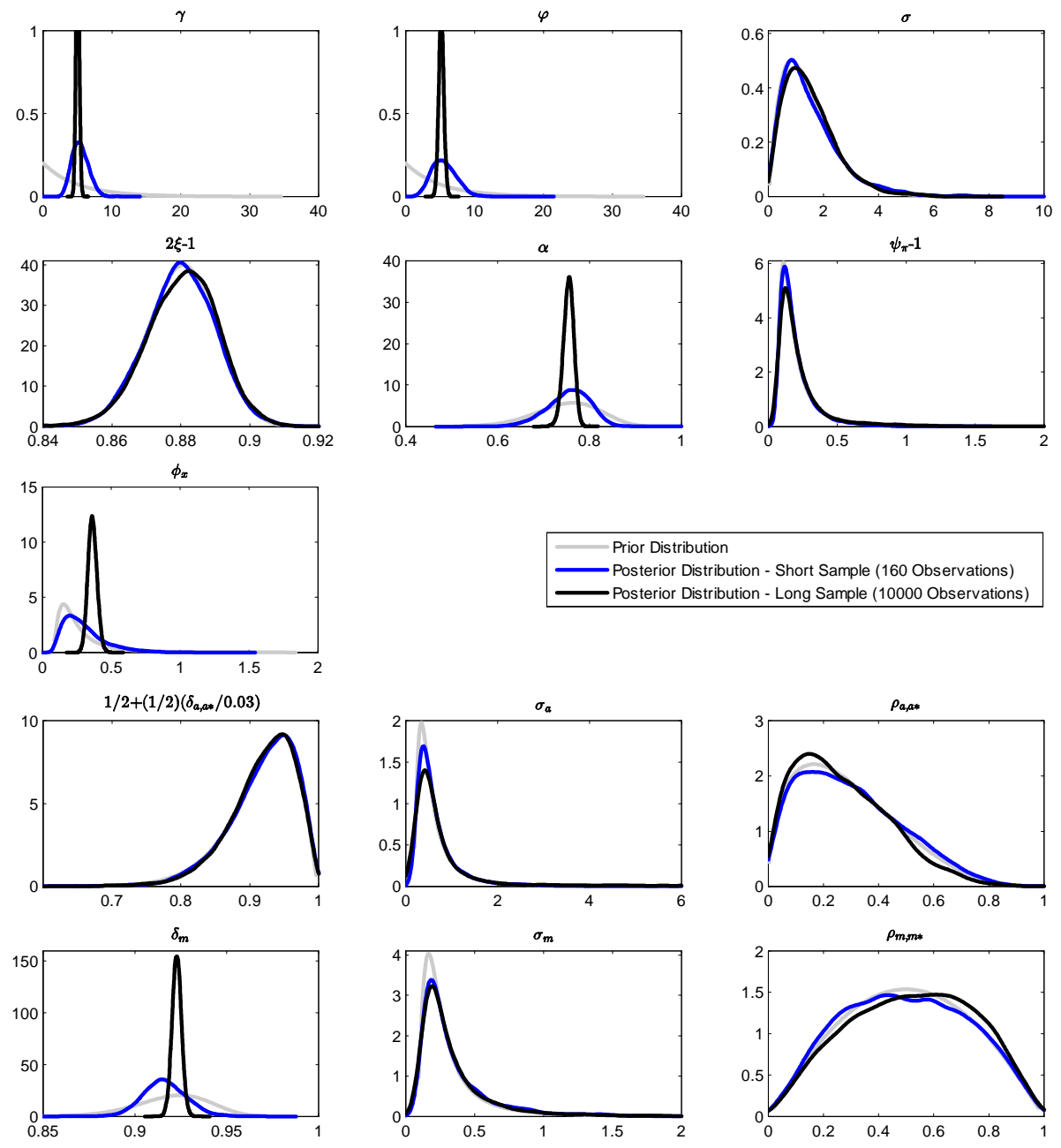

Note: The code is written for Matlab version 7.13.0.564. This figure plots the prior distributions of all 13 structural (policy and non-policy) and shock parameters that do not receive a degenerate prior distribution, It also includes the posterior distribution estimated from a short sample of 160 observations (S2) and the long sample of 10000 observations under the benchmark implementation based on four observables (Home and Foreign output, Home and Foreign inflation). The code for this chart is available upon request from the authors. 

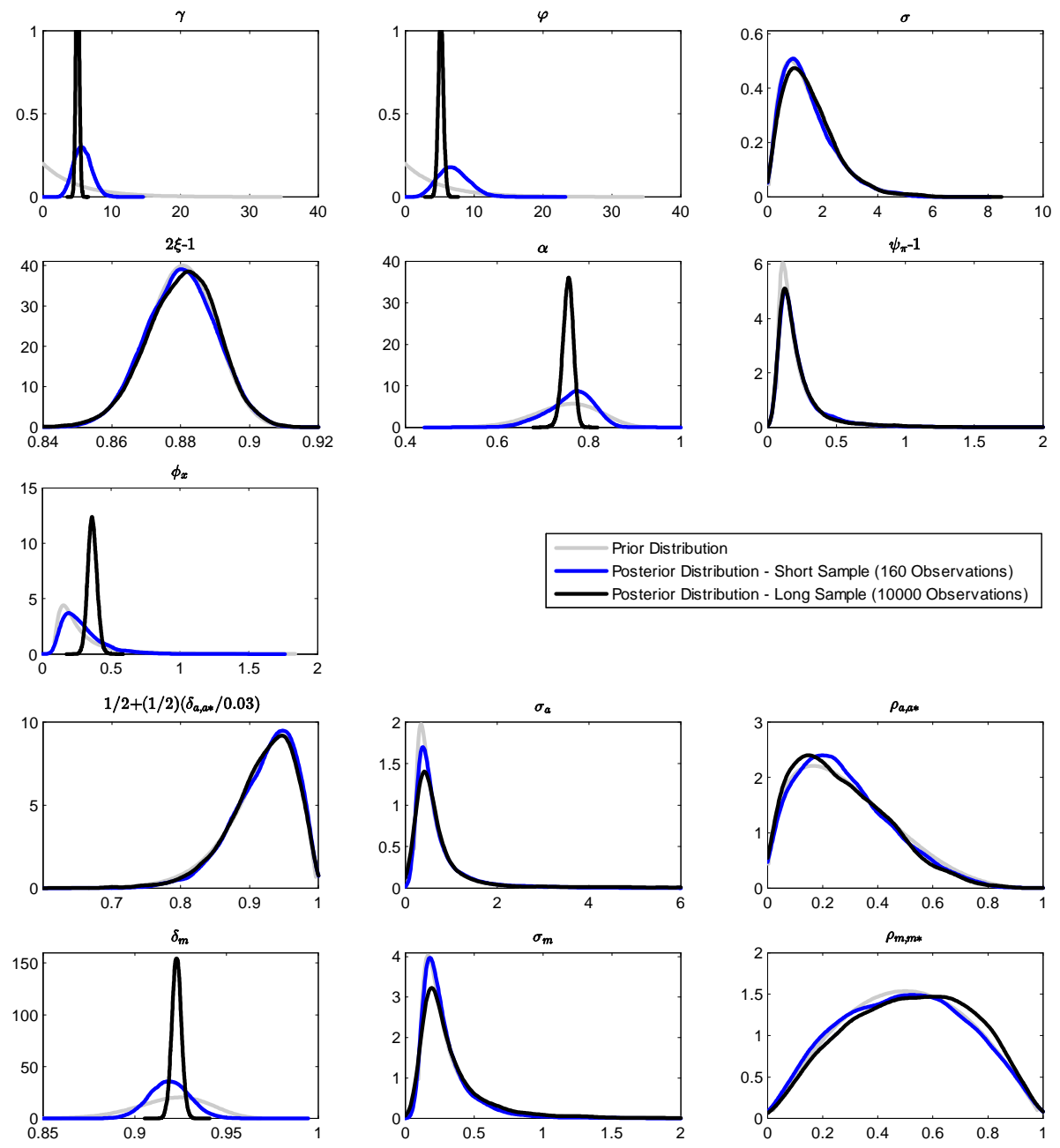

Note: The code is written for Matlab version 7.13.0.564. This figure plots the prior distributions of all 13 structural (policy and non-policy) and shock parameters that do not receive a degenerate prior distribution, It also includes the posterior distribution estimated from a short sample of 160 observations (S3) and the long sample of 10000 observations under the benchmark implementation based on four observables (Home and Foreign output, Home and Foreign inflation). The code for this chart is available upon request from the authors. 
FIGURE 7.A Prior and Posterior Distributions for the Structural and Shock Parameters - Short Sample, S1
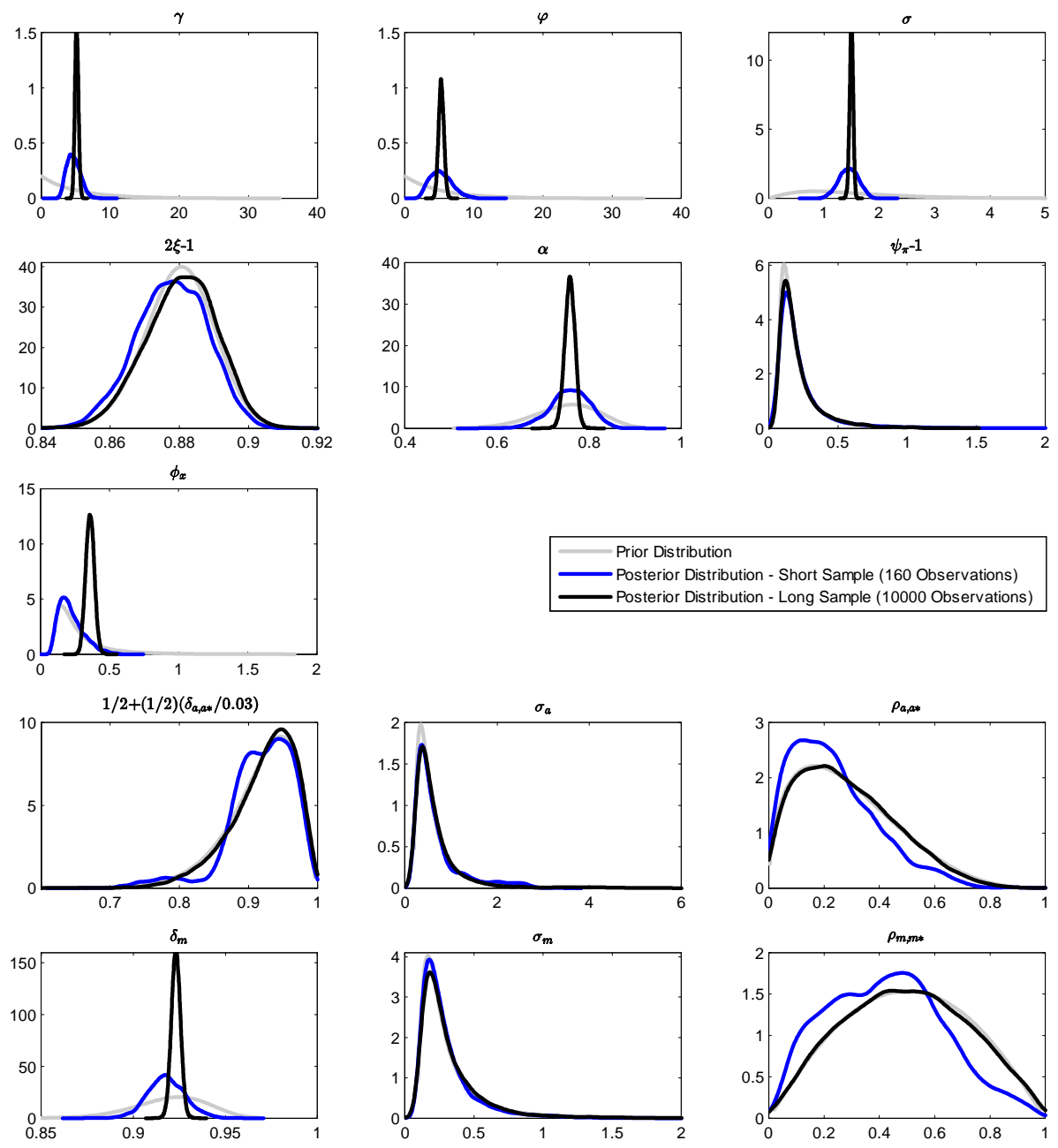

Note: The code is written for Matlab version 7.13.0.564. This figure plots the prior distributions of all 13 structural (policy and non-policy) and shock parameters that do not receive a degenerate prior distribution, It also includes the posterior distribution estimated from a short sample of 160 observations (S1) and the long sample of 10000 observations under the alternative implementation based on four observables (Home output, Home and Foreign inflation, and terms of trade). The code for this chart is available upon request from the authors. 

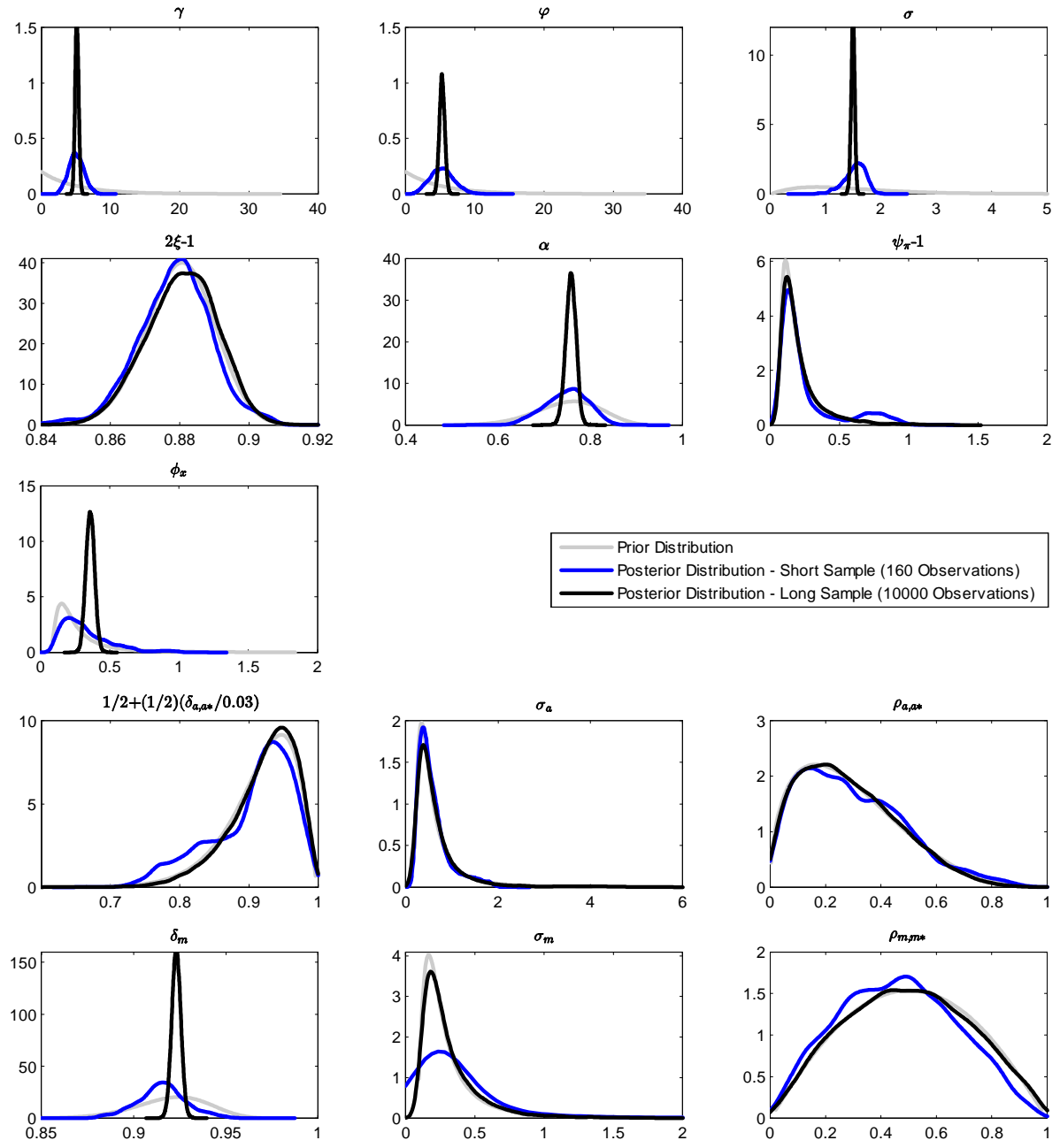

Note: The code is written for Matlab version 7.13.0.564. This figure plots the prior distributions of all 13 structural (policy and non-policy) and shock parameters that do not receive a degenerate prior distribution, It also includes the posterior distribution estimated from a short sample of 160 observations (S2) and the long sample of 10000 observations under the alternative implementation based on four observables (Home output, Home and Foreign inflation, and terms of trade). The code for this chart is available upon request from the authors. 

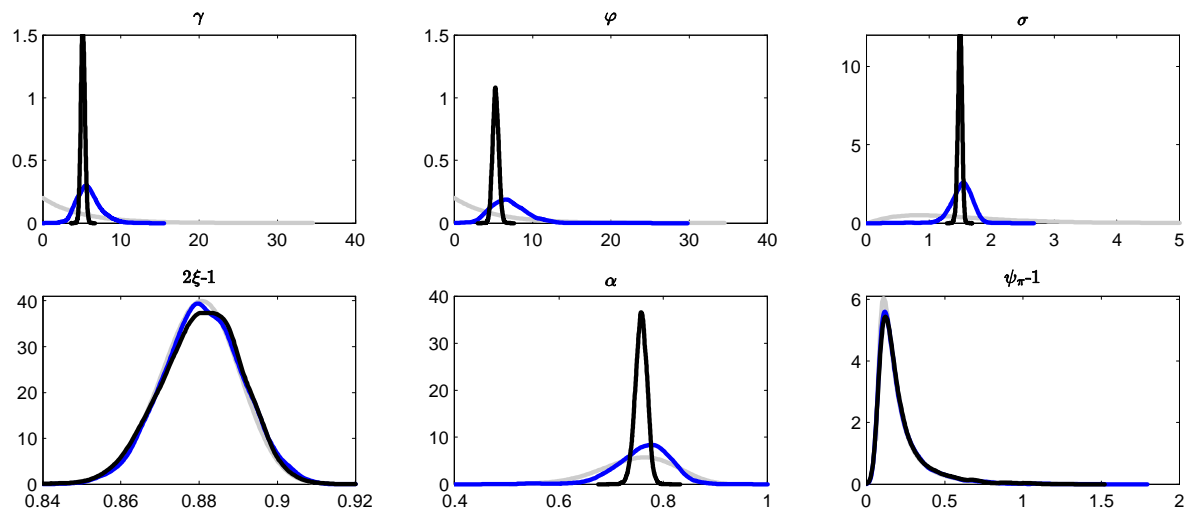

$\phi_{x}$

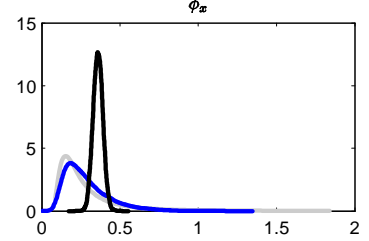

- Posterior Distribution - Short Sample (160 Observations) Posterior Distribution - Long Sample (10000 Observations)
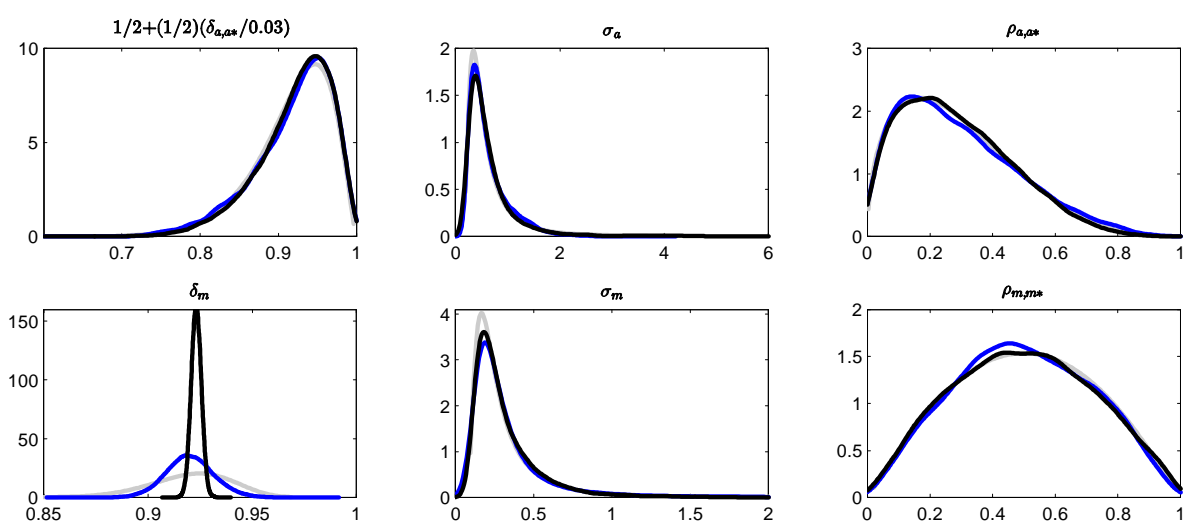

Note: The code is written for Matlab version 7.13.0.564. This figure plots the prior distributions of all 13 structural (policy and non-policy) and shock parameters that do not receive a degenerate prior distribution, It also includes the posterior distribution estimated from a short sample of 160 observations (S3) and the long sample of 10000 observations under the alternative implementation based on four observables (Home output, Home and Foreign inflation, and terms of trade). The code for this chart is available upon request from the authors. 

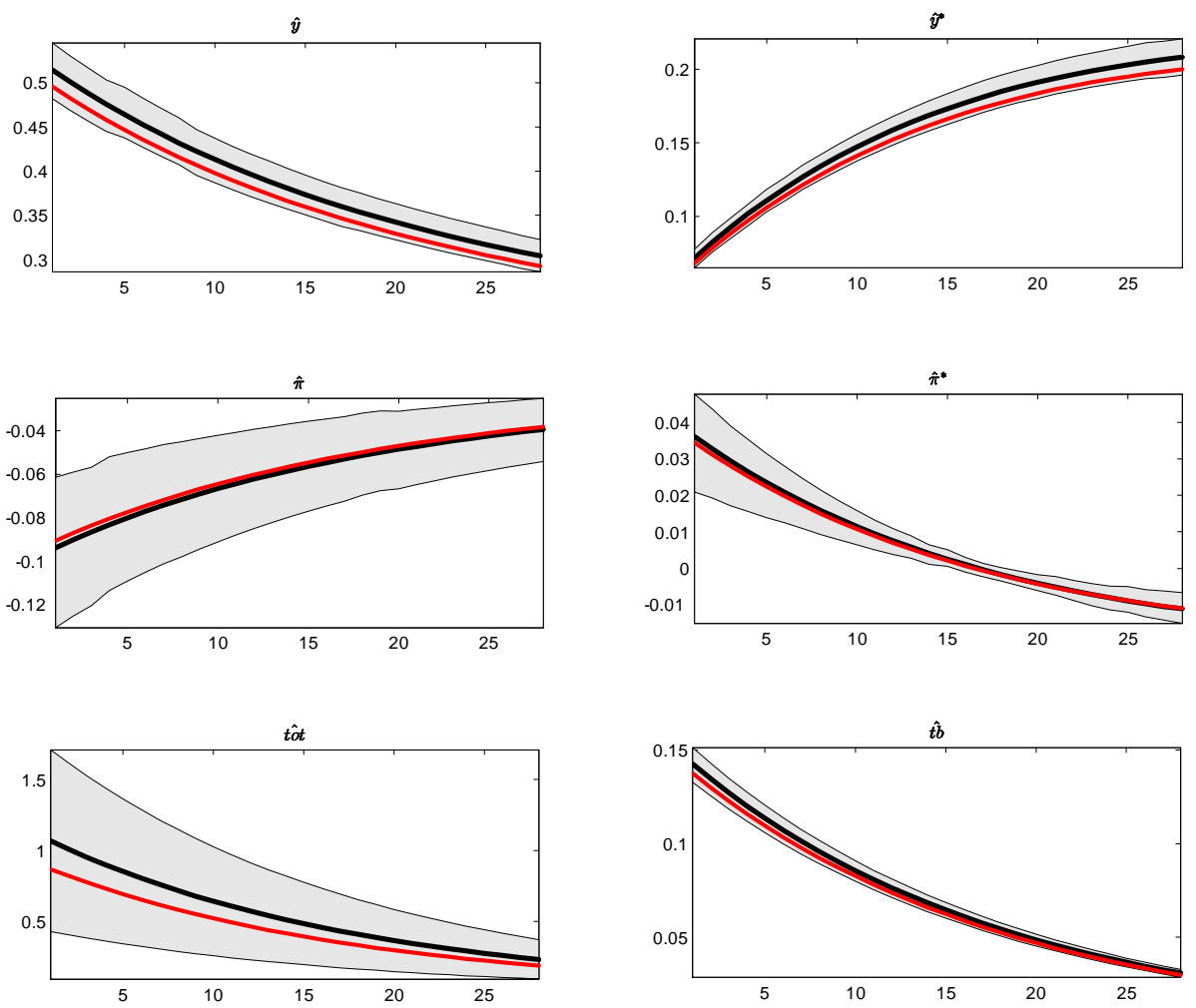

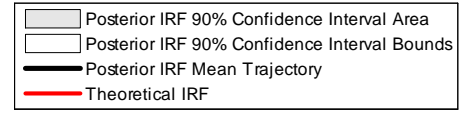

Note: The code is written for Matlab version 7.13.0.564. This figure plots the Bayesian impulse response functions (IRFs) for Home output, Foreign output, Home inflation, Foreign inflation, terms of trade and the trade balance in response to Home productivity shock innovations. The IRFs are estimated from a short sample of 160 observations (S1) under the benchmark implementation of the model based on four observables (Home and Foreign output, Home and Foreign inflation). The code for this chart is available upon request from the authors. 

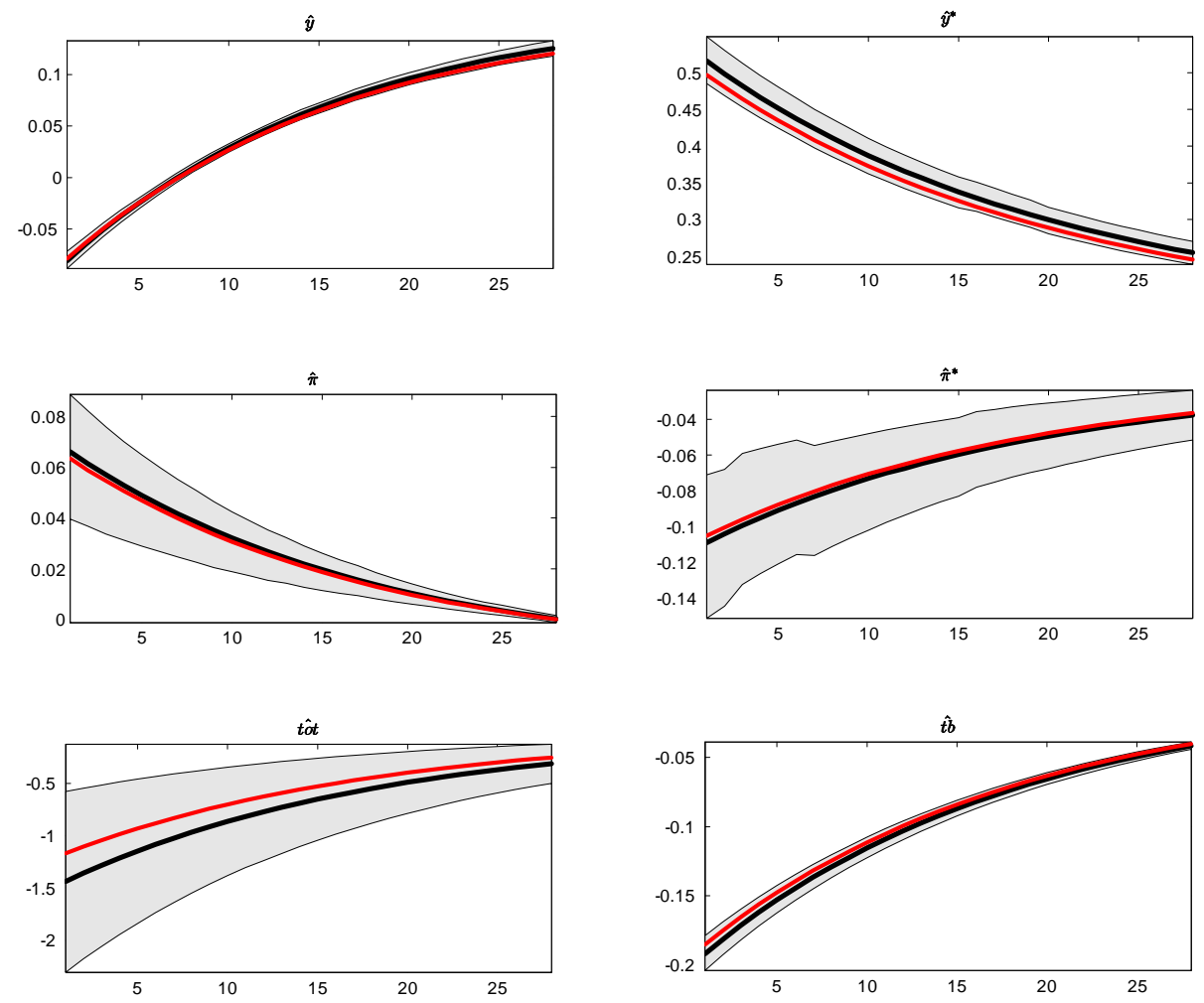

\begin{tabular}{|c|c|}
\hline & $\begin{array}{l}\text { Posterior IRF 90\% Confidence Interval Area } \\
\text { Posterior IRF } 90 \% \text { Confidence Interval Bounds }\end{array}$ \\
\hline & $\begin{array}{l}\text { Posterior IRF Mean Trajectory } \\
\text { Theoretical IRF }\end{array}$ \\
\hline
\end{tabular}

Note: The code is written for Matlab version 7.13.0.564. This figure plots the Bayesian impulse response functions (IRFs) for Home output, Foreign output, Home inflation, Foreign inflation, terms of trade and the trade balance in response to Foreign productivity shock innovations. The IRFs are estimated from a short sample of 160 observations (S1) under the benchmark implementation of the model based on four observables (Home and Foreign output, Home and Foreign inflation). The code for this chart is available upon request from the authors. 

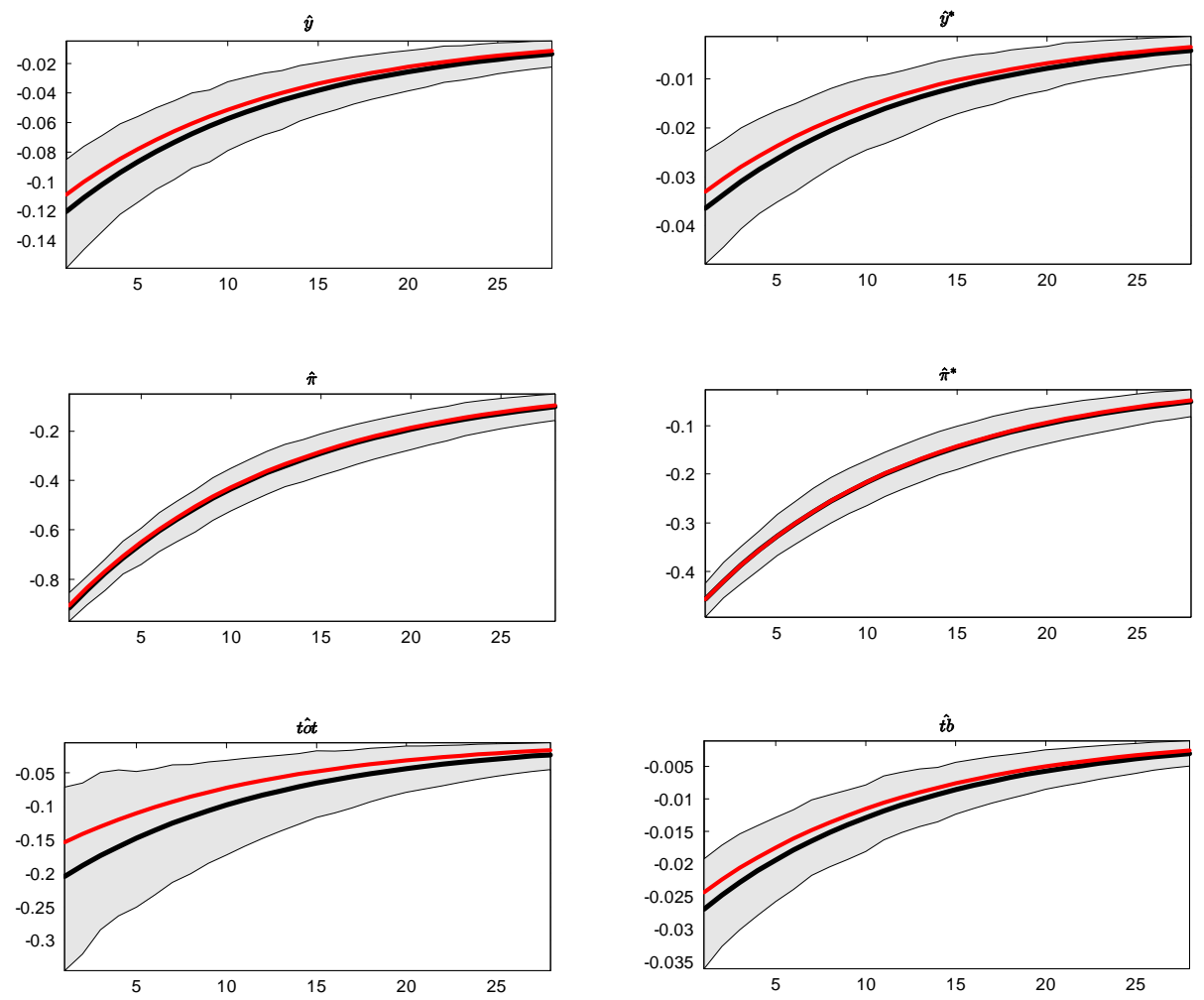

\begin{tabular}{|c|c|}
\hline & $\begin{array}{l}\text { Posterior IRF } 90 \% \text { Confidence Interval Area } \\
\text { Posterior IRF } 90 \% \text { Confidence Interval Bounds }\end{array}$ \\
\hline & $\begin{array}{l}\text {-Posterior IRF Mean Trajectory } \\
\text { - Theoretical IRF }\end{array}$ \\
\hline
\end{tabular}

Note: The code is written for Matlab version 7.13.0.564. This figure plots the Bayesian impulse response functions (IRFs) for Home output, Foreign output, Home inflation, Foreign inflation, terms of trade and the trade balance in response to Home monetary shock innovations. The IRFs are estimated from a short sample of 160 observations (S1) under the benchmark implementation of the model based on four observables (Home and Foreign output, Home and Foreign inflation). The code for this chart is available upon request from the authors. 

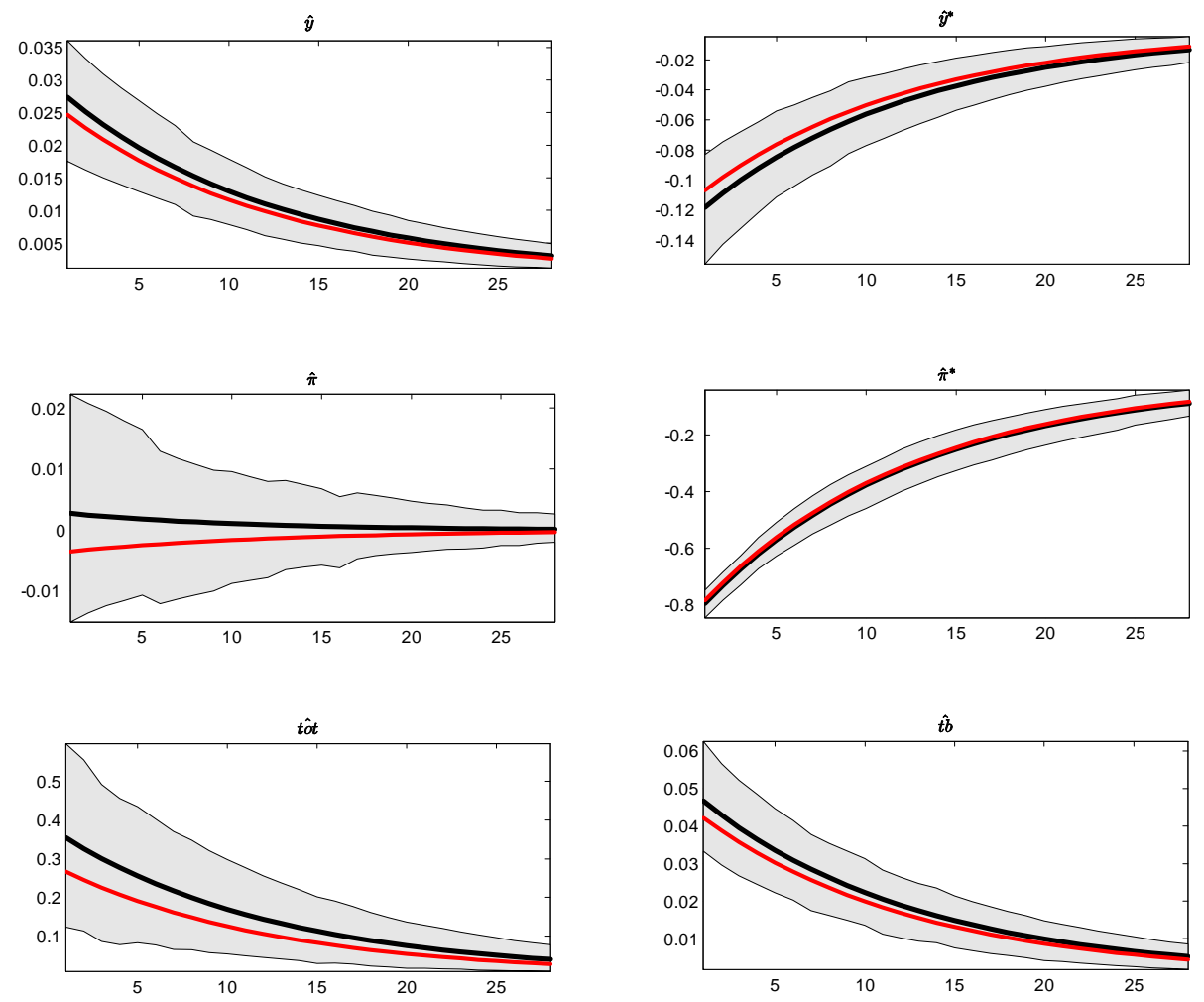

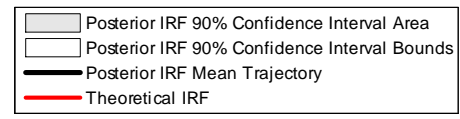

Note: The code is written for Matlab version 7.13.0.564. This figure plots the Bayesian impulse response functions (IRFs) for Home output, Foreign output, Home inflation, Foreign inflation, terms of trade and the trade balance in response to Foreign monetary shock innovations. The IRFs are estimated from a short sample of 160 observations (S1) under the benchmark implementation of the model based on four observables (Home and Foreign output, Home and Foreign inflation). The code for this chart is available upon request from the authors. 

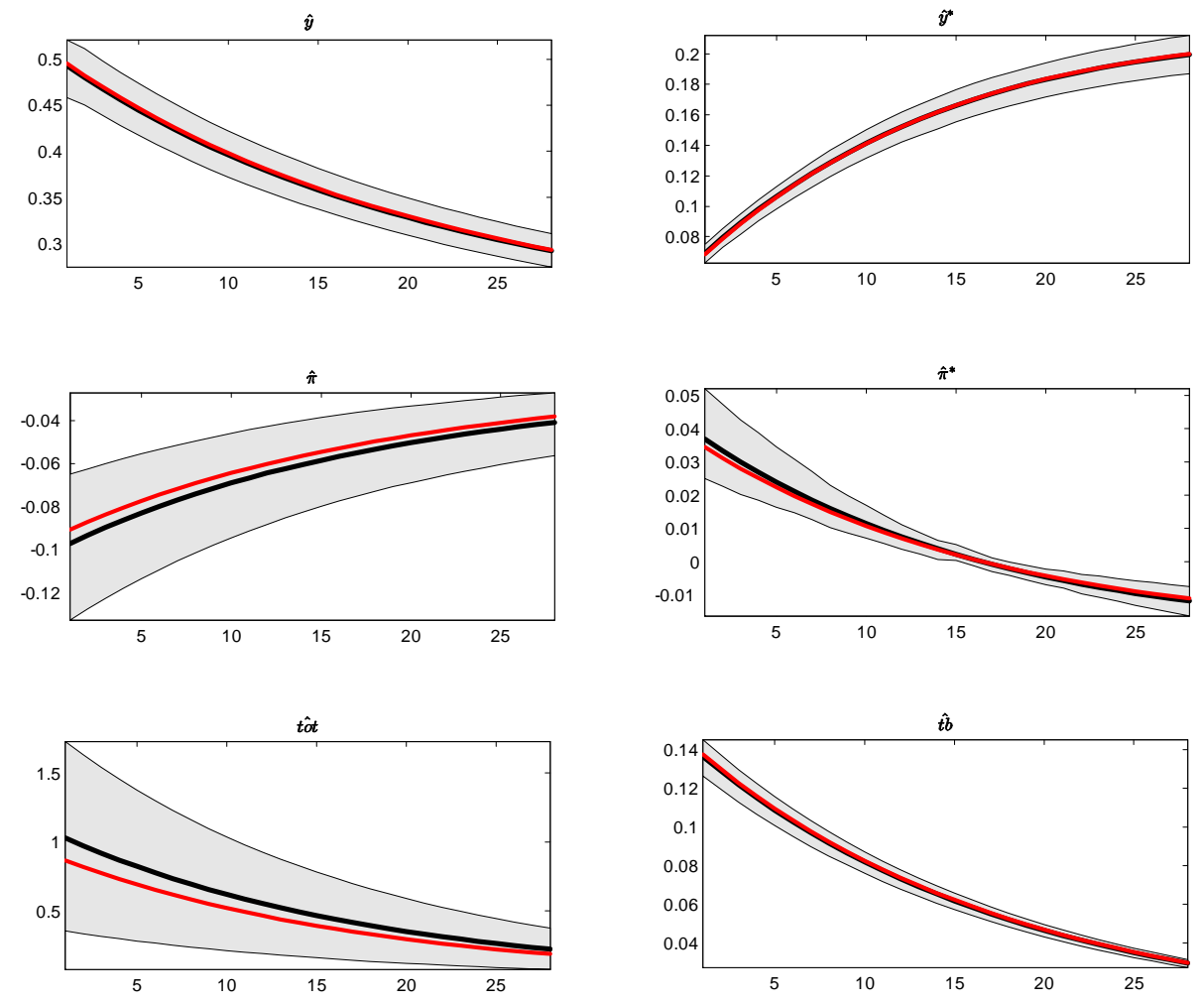

\begin{tabular}{|c|c|}
\hline L & Posterior IRF $90 \%$ Confidence Interval Area \\
\hline & Posterior IRF $90 \%$ Confidence Interval Bounds \\
\hline & $\begin{array}{l}\text { - Posterior IRF Mean Trajectory } \\
\text {-Theoretical IRF }\end{array}$ \\
\hline
\end{tabular}

Note: The code is written for Matlab version 7.13.0.564. This figure plots the Bayesian impulse response functions (IRFs) for Home output, Foreign output, Home inflation, Foreign inflation, terms of trade and the trade balance in response to Home productivity shock innovations. The IRFs are estimated from a short sample of 160 observations (S2) under the benchmark implementation of the model based on four observables (Home and Foreign output, Home and Foreign inflation). The code for this chart is available upon request from the authors. 

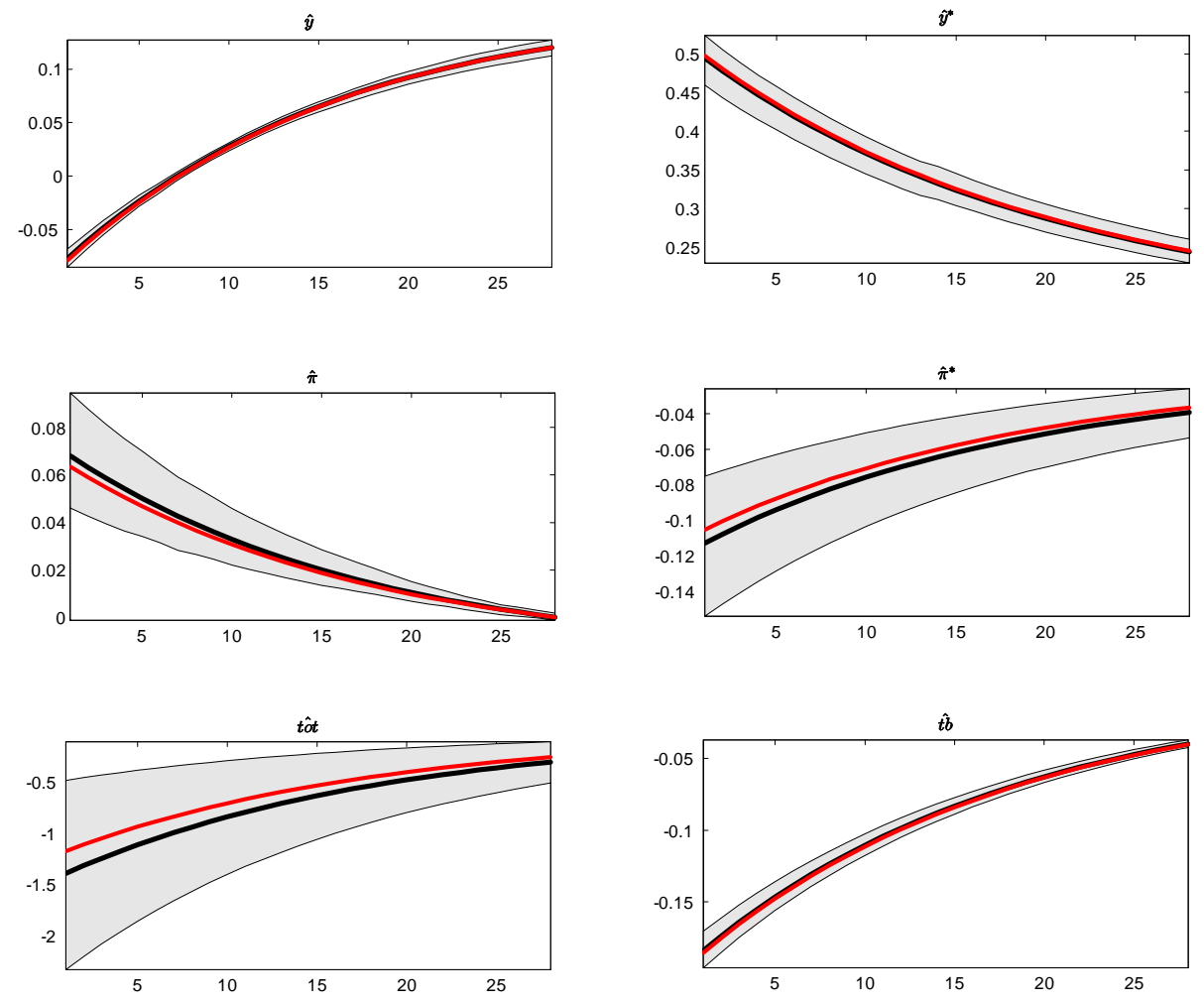

\begin{tabular}{|c|c|}
\hline & $\begin{array}{l}\text { Posterior IRF } 90 \% \text { Confidence Interval Area } \\
\text { Posterior IRF } 90 \% \text { Confidence Interval Bounds }\end{array}$ \\
\hline & $\begin{array}{l}\text {-Posterior IRF Mean Trajectory } \\
\text { - Theoretical IRF }\end{array}$ \\
\hline
\end{tabular}

Note: The code is written for Matlab version 7.13.0.564. This figure plots the Bayesian impulse response functions (IRFs) for Home output, Foreign output, Home inflation, Foreign inflation, terms of trade and the trade balance in response to Foreign productivity shock innovations. The IRFs are estimated from a short sample of 160 observations (S2) under the benchmark implementation of the model based on four observables (Home and Foreign output, Home and Foreign inflation). The code for this chart is available upon request from the authors. 

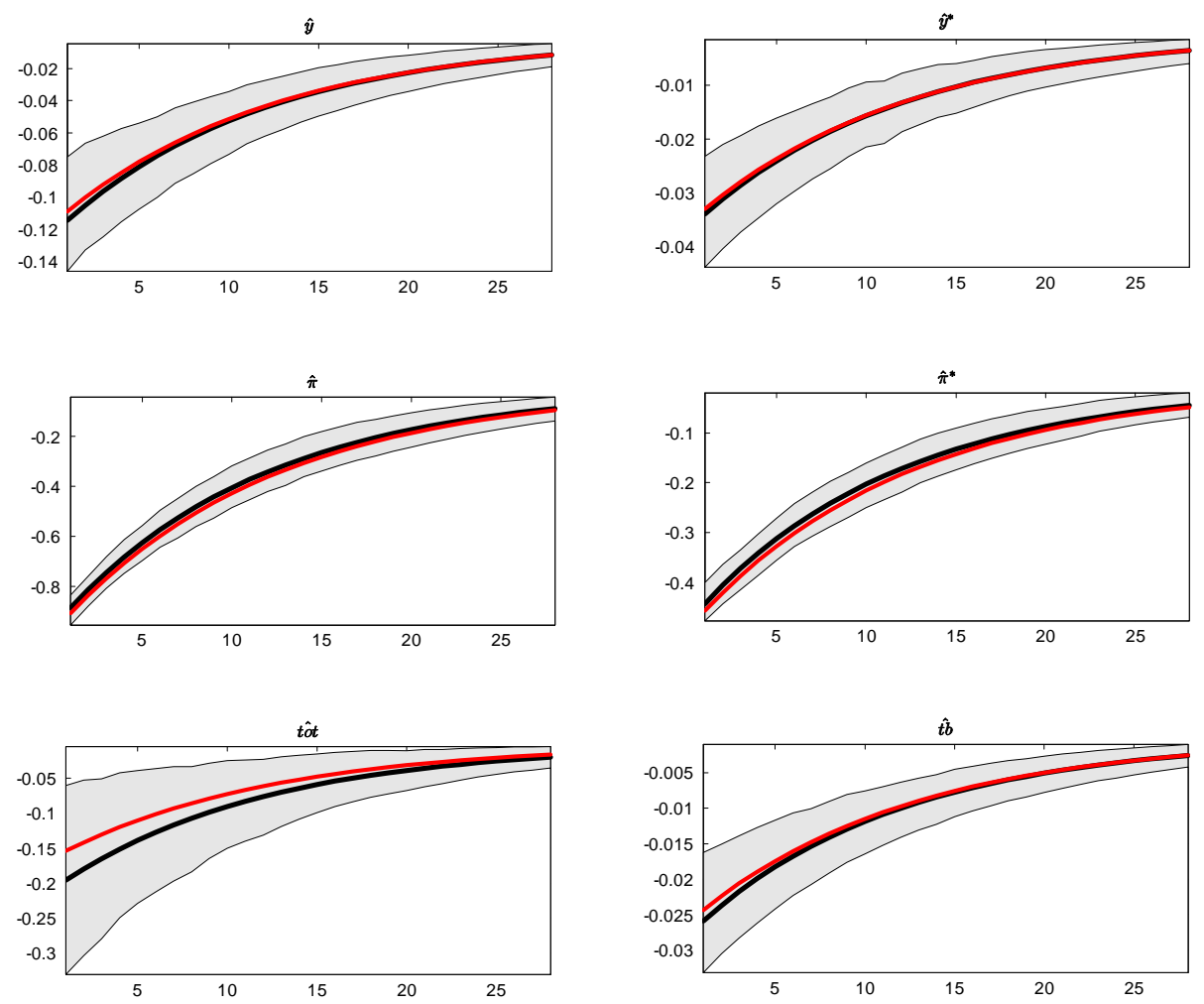

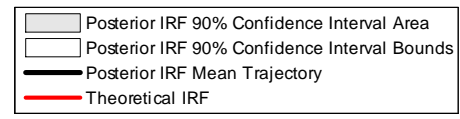

Note: The code is written for Matlab version 7.13.0.564. This figure plots the Bayesian impulse response functions (IRFs) for Home output, Foreign output, Home inflation, Foreign inflation, terms of trade and the trade balance in response to Home monetary shock innovations. The IRFs are estimated from a short sample of 160 observations (S2) under the benchmark implementation of the model based on four observables (Home and Foreign output, Home and Foreign inflation). The code for this chart is available upon request from the authors. 

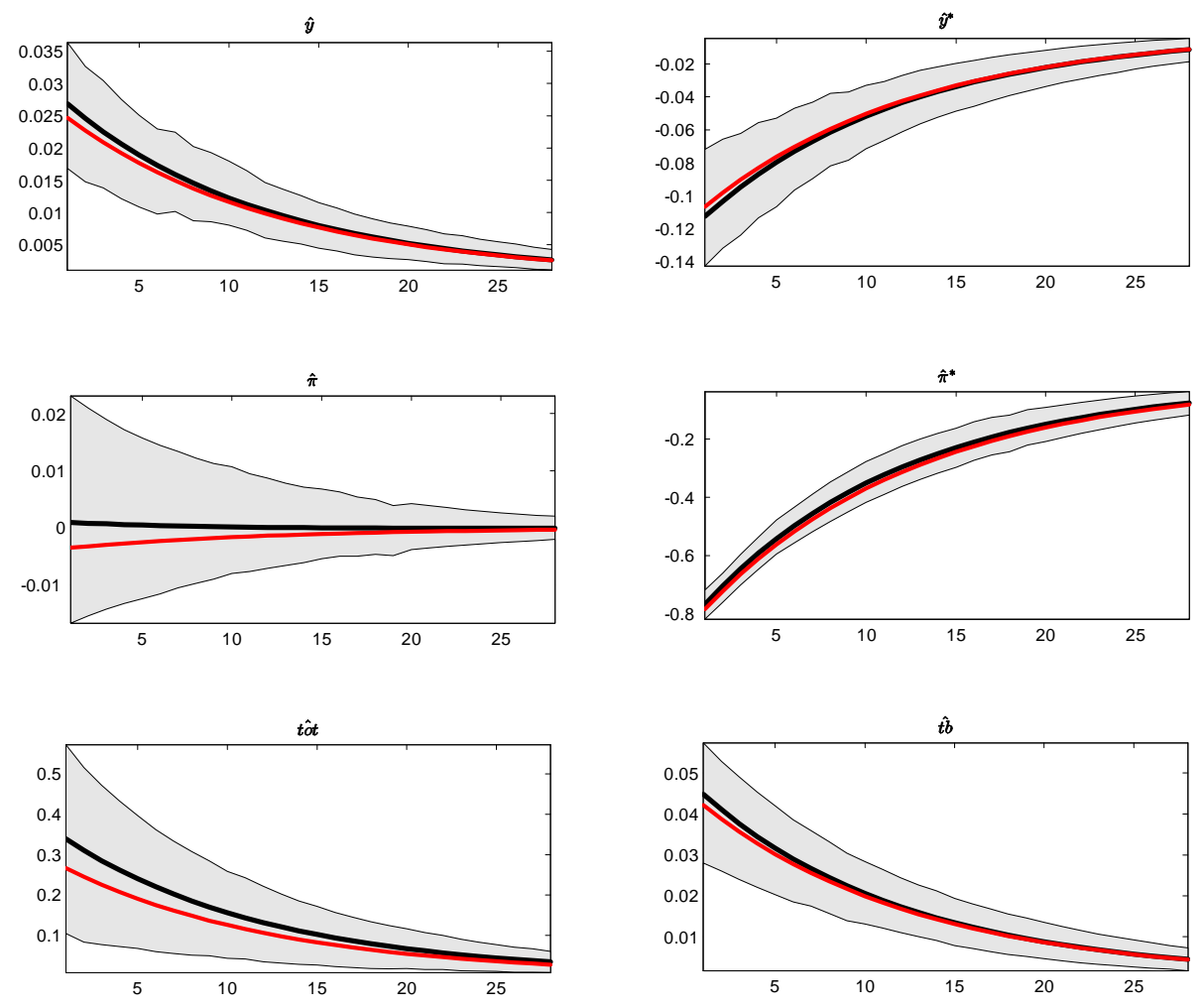

\begin{tabular}{|c|c|}
\hline & Posterior IRF $90 \%$ Confidence Interval Area \\
\hline & ] Posterior IRF $90 \%$ Confidence Interval Bounds \\
\hline & $\begin{array}{l}\text { Posterior IRF Mean Trajectory } \\
\text { - Theoretical IRF }\end{array}$ \\
\hline
\end{tabular}

Note: The code is written for Matlab version 7.13.0.564. This figure plots the Bayesian impulse response functions (IRFs) for Home output, Foreign output, Home inflation, Foreign inflation, terms of trade and the trade balance in response to Foreign monetary shock innovations. The IRFs are estimated from a short sample of 160 observations (S2) under the benchmark implementation of the model based on four observables (Home and Foreign output, Home and Foreign inflation). The code for this chart is available upon request from the authors. 

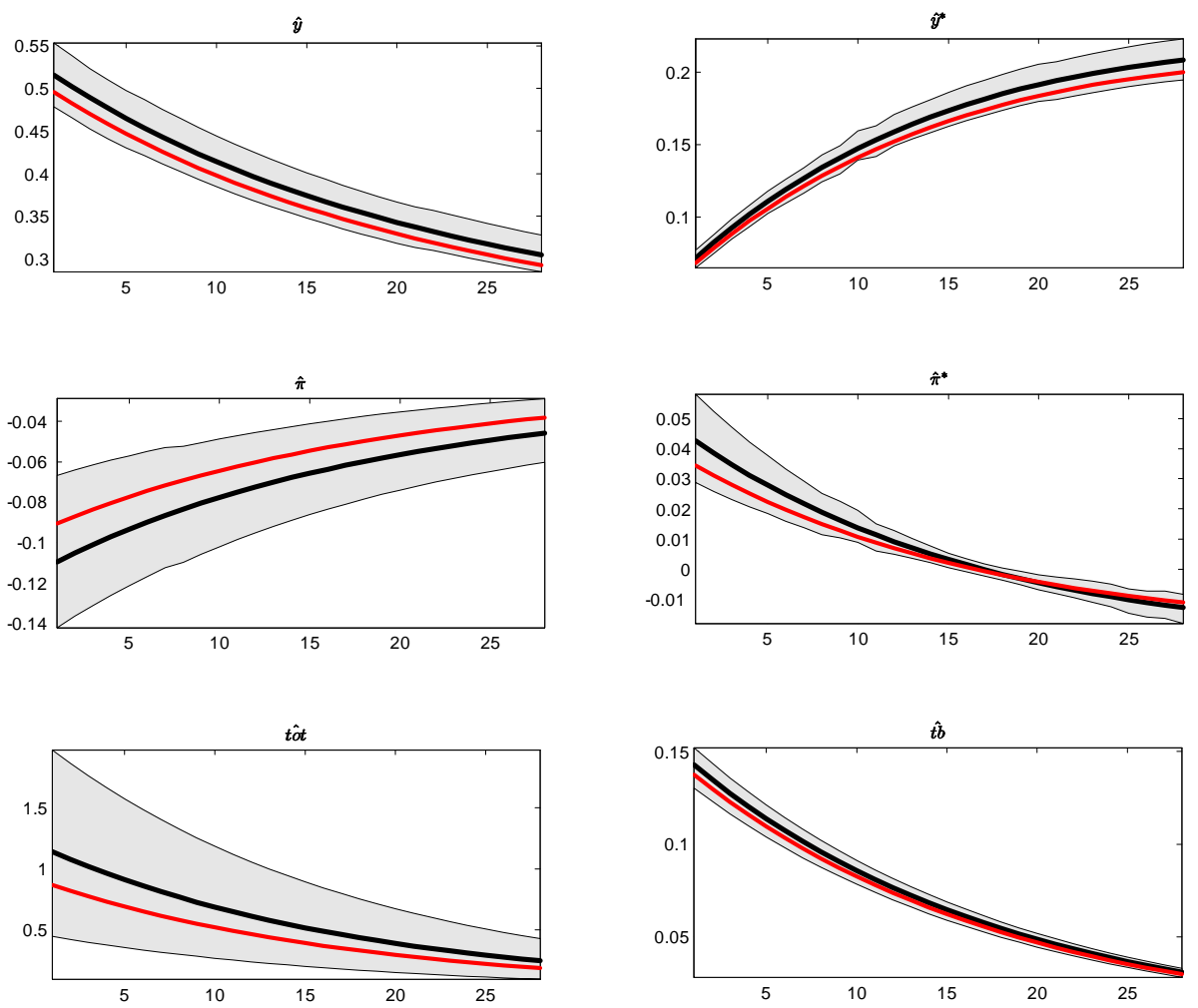

\begin{tabular}{|c|c|}
\hline & Posterior IRF 90\% Confidence Interval Area \\
\hline & Posterior IRF $90 \%$ Confidence Interval Bounds \\
\hline & $\begin{array}{l}\text { Posterior IRF Mean Trajectory } \\
\text {-Theoretical IRF }\end{array}$ \\
\hline
\end{tabular}

Note: The code is written for Matlab version 7.13.0.564. This figure plots the Bayesian impulse response functions (IRFs) for Home output, Foreign output, Home inflation, Foreign inflation, terms of trade and the trade balance in response to Home productivity shock innovations. The IRFs are estimated from a short sample of 160 observations (S3) under the benchmark implementation of the model based on four observables (Home and Foreign output, Home and Foreign inflation). The code for this chart is available upon request from the authors. 

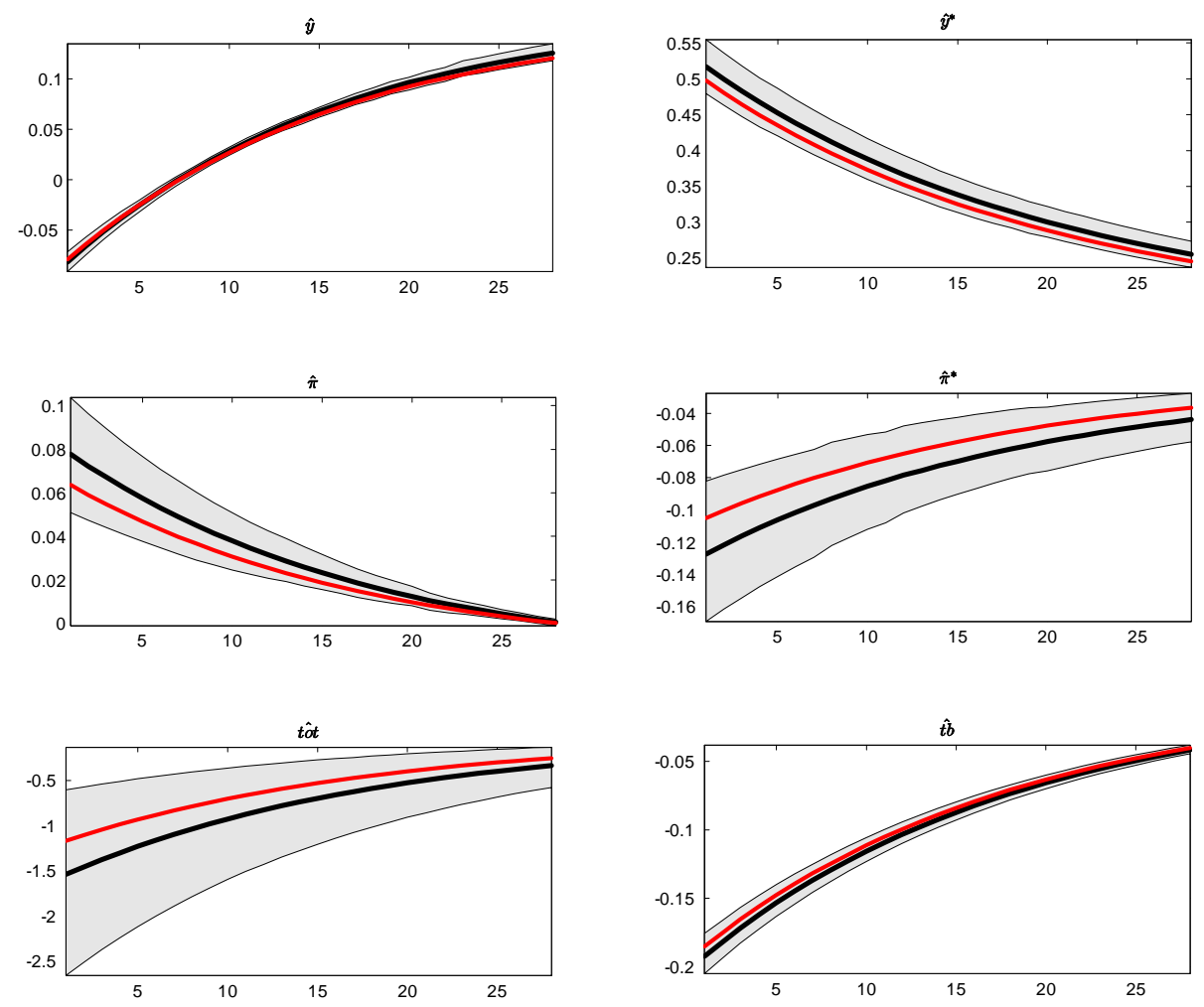

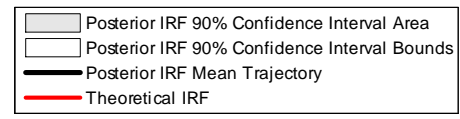

Note: The code is written for Matlab version 7.13.0.564. This figure plots the Bayesian impulse response functions (IRFs) for Home output, Foreign output, Home inflation, Foreign inflation, terms of trade and the trade balance in response to Foreign productivity shock innovations. The IRFs are estimated from a short sample of 160 observations (S3) under the benchmark implementation of the model based on four observables (Home and Foreign output, Home and Foreign inflation). The code for this chart is available upon request from the authors. 

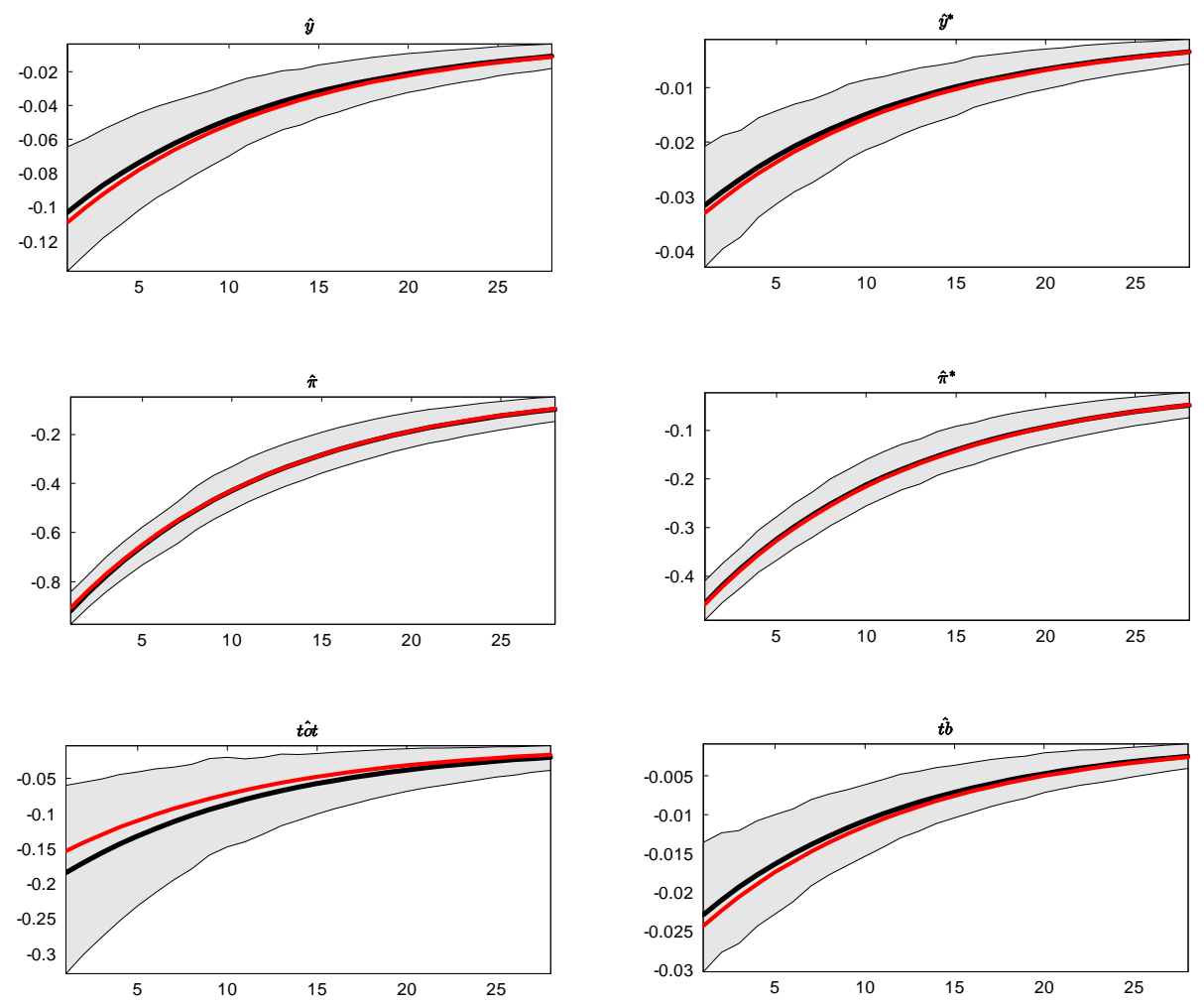

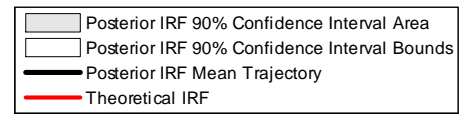

Note: The code is written for Matlab version 7.13.0.564. This figure plots the Bayesian impulse response functions (IRFs) for Home output, Foreign output, Home inflation, Foreign inflation, terms of trade and the trade balance in response to Home monetary shock innovations. The IRFs are estimated from a short sample of 160 observations (S3) under the benchmark implementation of the model based on four observables (Home and Foreign output, Home and Foreign inflation). The code for this chart is available upon request from the authors. 
FIGURE 8.C Bayesian IRFs With Respect To Orthogonalized Foreign Monetary Shock Innovations - Short Sample, S3
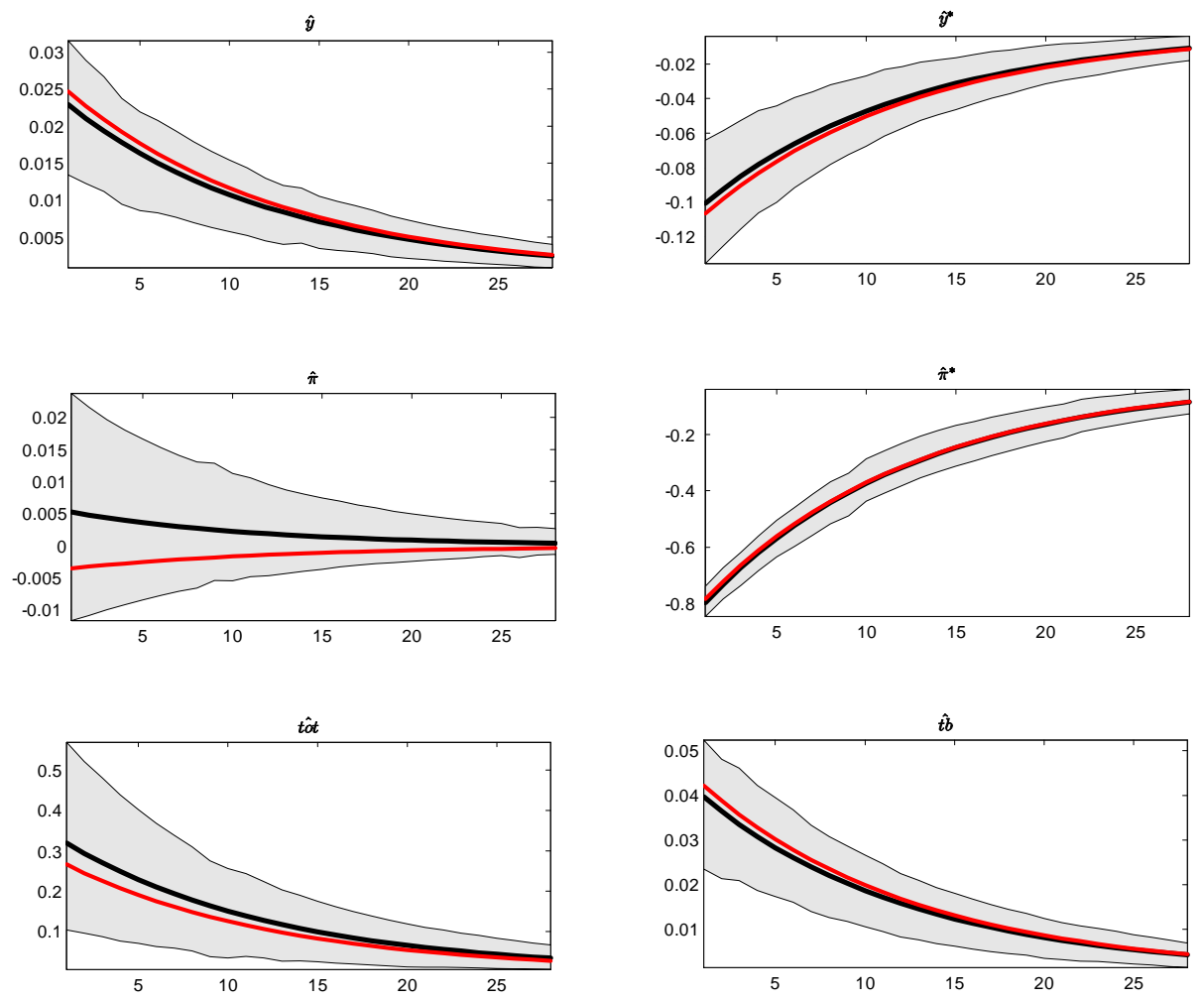

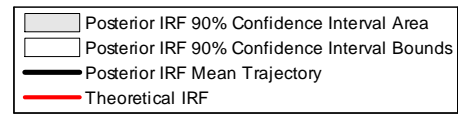

Note: The code is written for Matlab version 7.13.0.564. This figure plots the Bayesian impulse response functions (IRFs) for Home output, Foreign output, Home inflation, Foreign inflation, terms of trade and the trade balance in response to Foreign monetary shock innovations. The IRFs are estimated from a short sample of 160 observations (S3) under the benchmark implementation of the model based on four observables (Home and Foreign output, Home and Foreign inflation). The code for this chart is available upon request from the authors. 

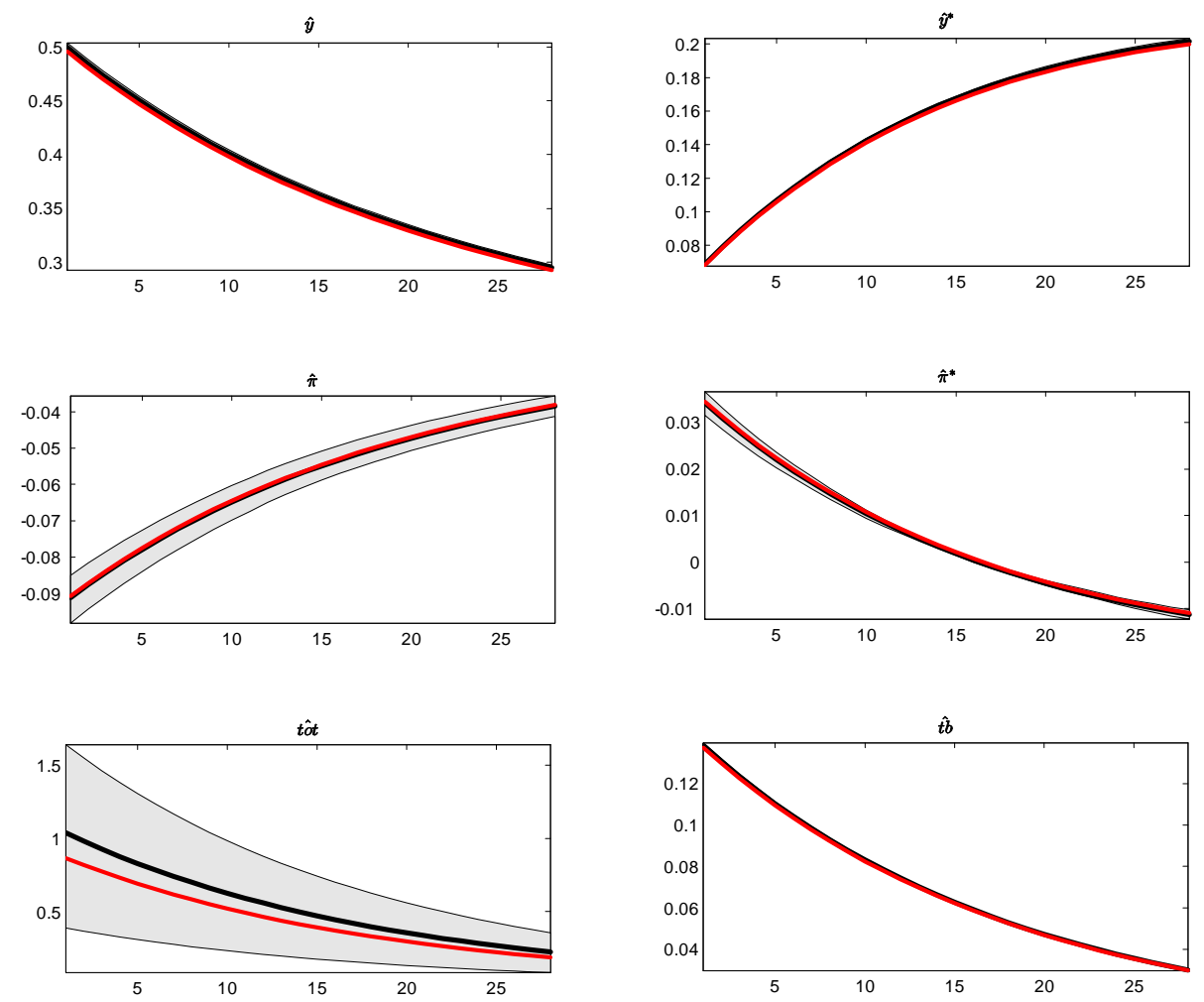

\begin{tabular}{|c|c|}
\hline & $\begin{array}{l}\text { Posterior IRF } 90 \% \text { Confidence Interval Area } \\
\text { Posterior IRF } 90 \% \text { Confidence Interval Bounds }\end{array}$ \\
\hline & $\begin{array}{l}\text {-Posterior IRF Mean Trajectory } \\
\text { - Theoretical IRF }\end{array}$ \\
\hline
\end{tabular}

Note: The code is written for Matlab version 7.13.0.564. This figure plots the Bayesian impulse response functions (IRFs) for Home output, Foreign output, Home inflation, Foreign inflation, terms of trade and the trade balance in response to Home productivity shock innovations. The IRFs are estimated from the long sample of 10000 observations under the benchmark implementation of the model based on four observables (Home and Foreign output, Home and Foreign inflation). The code for this chart is available upon request from the authors. 

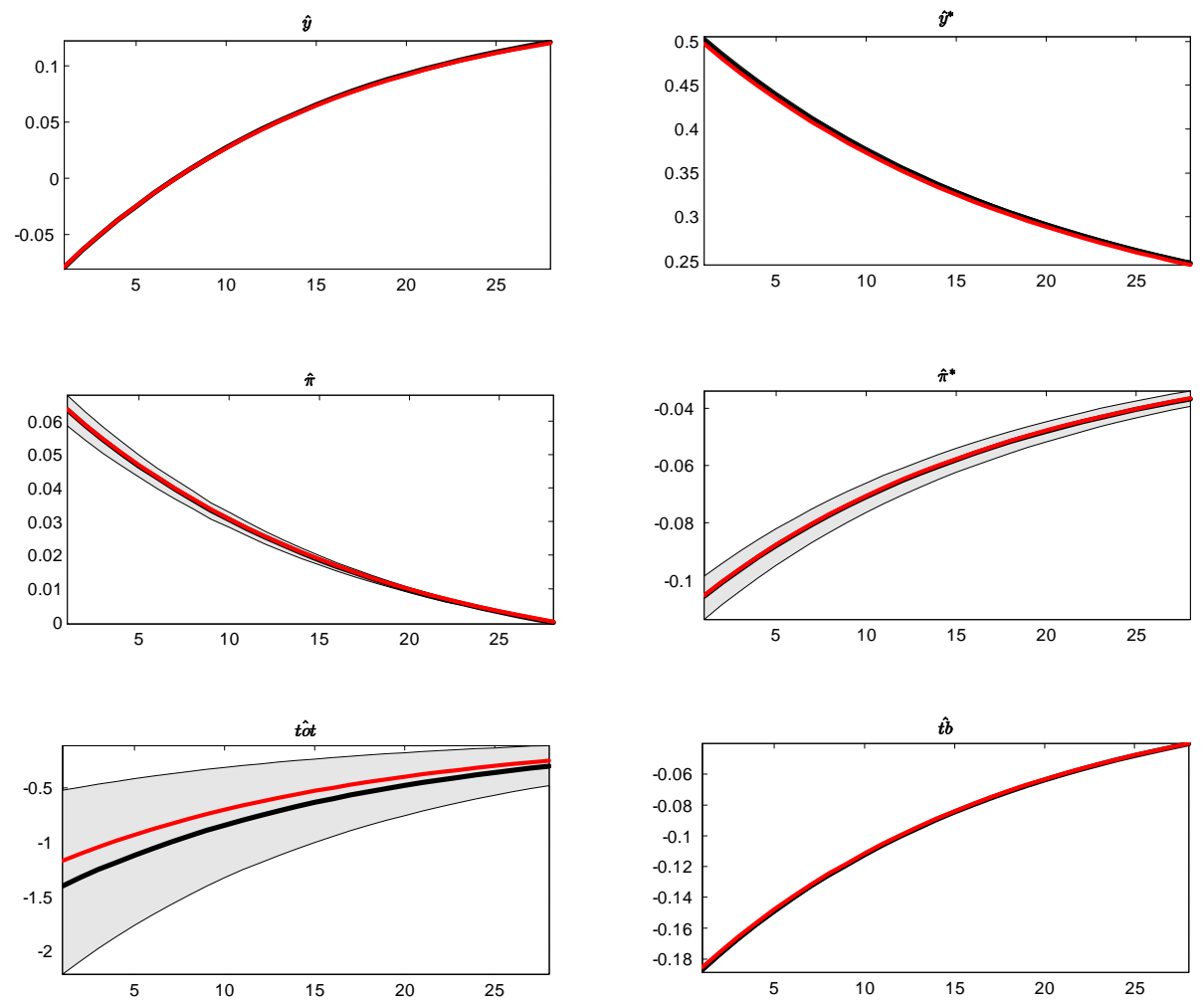

\begin{tabular}{|c|c|}
\hline & Posterior IRF 90\% Confidence Interval Area \\
\hline & Posterior IRF $90 \%$ Confidence Interval Bounds \\
\hline & Posterior IRF Mean Trajectory \\
\hline
\end{tabular}

Note: The code is written for Matlab version 7.13.0.564. This figure plots the Bayesian impulse response functions (IRFs) for Home output, Foreign output, Home inflation, Foreign inflation, terms of trade and the trade balance in response to Foreign productivity shock innovations. The IRFs are estimated from the long sample of 10000 observations under the benchmark implementation of the model based on four observables (Home and Foreign output, Home and Foreign inflation). The code for this chart is available upon request from the authors. 

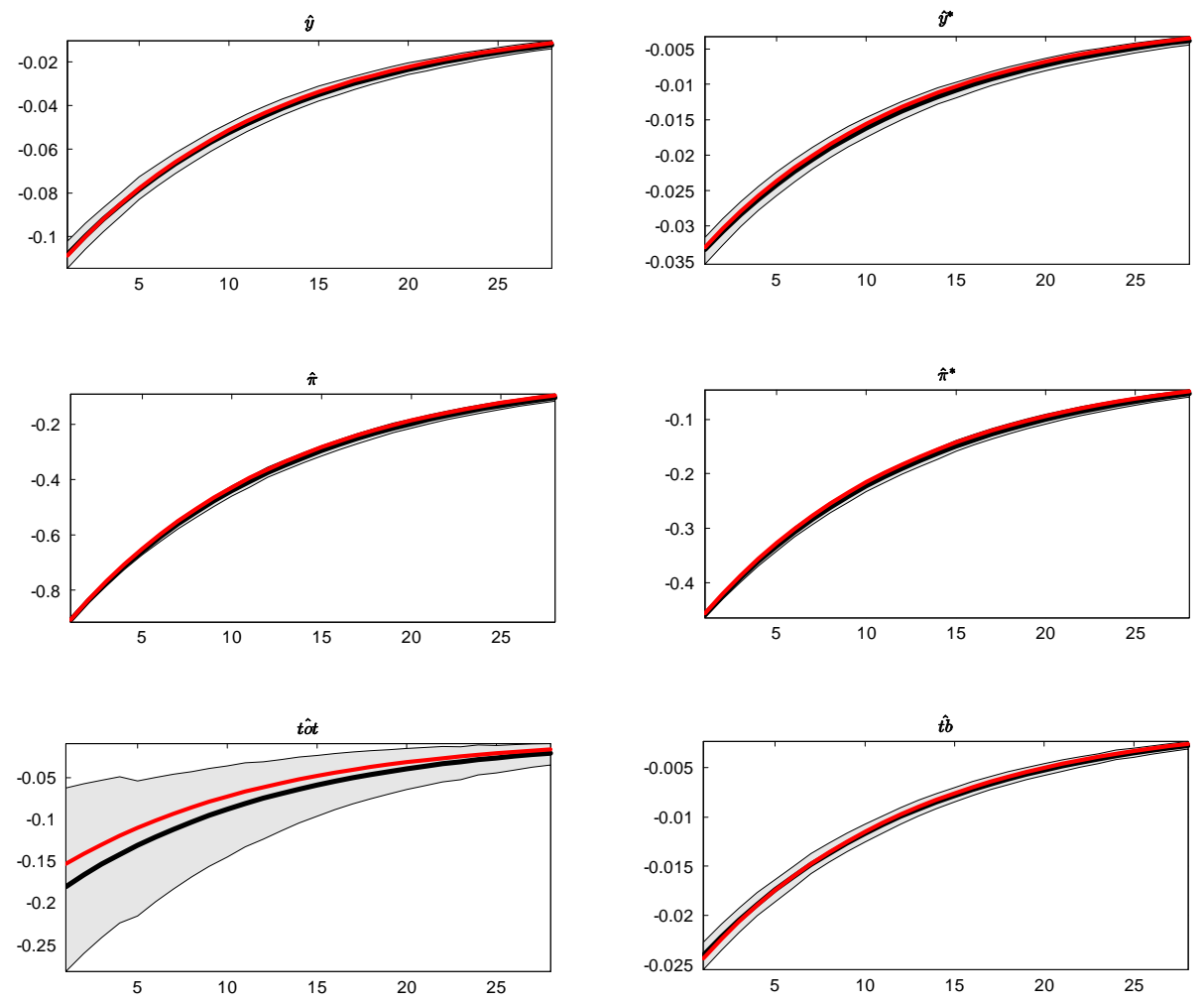

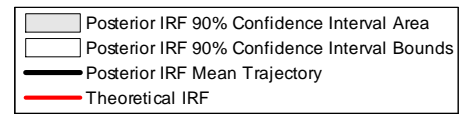

Note: The code is written for Matlab version 7.13.0.564. This figure plots the Bayesian impulse response functions (IRFs) for Home output, Foreign output, Home inflation, Foreign inflation, terms of trade and the trade balance in response to Home monetary shock innovations. The IRFs are estimated from the long sample of 10000 observations under the benchmark implementation of the model based on four observables (Home and Foreign output, Home and Foreign inflation). The code for this chart is available upon request from the authors. 

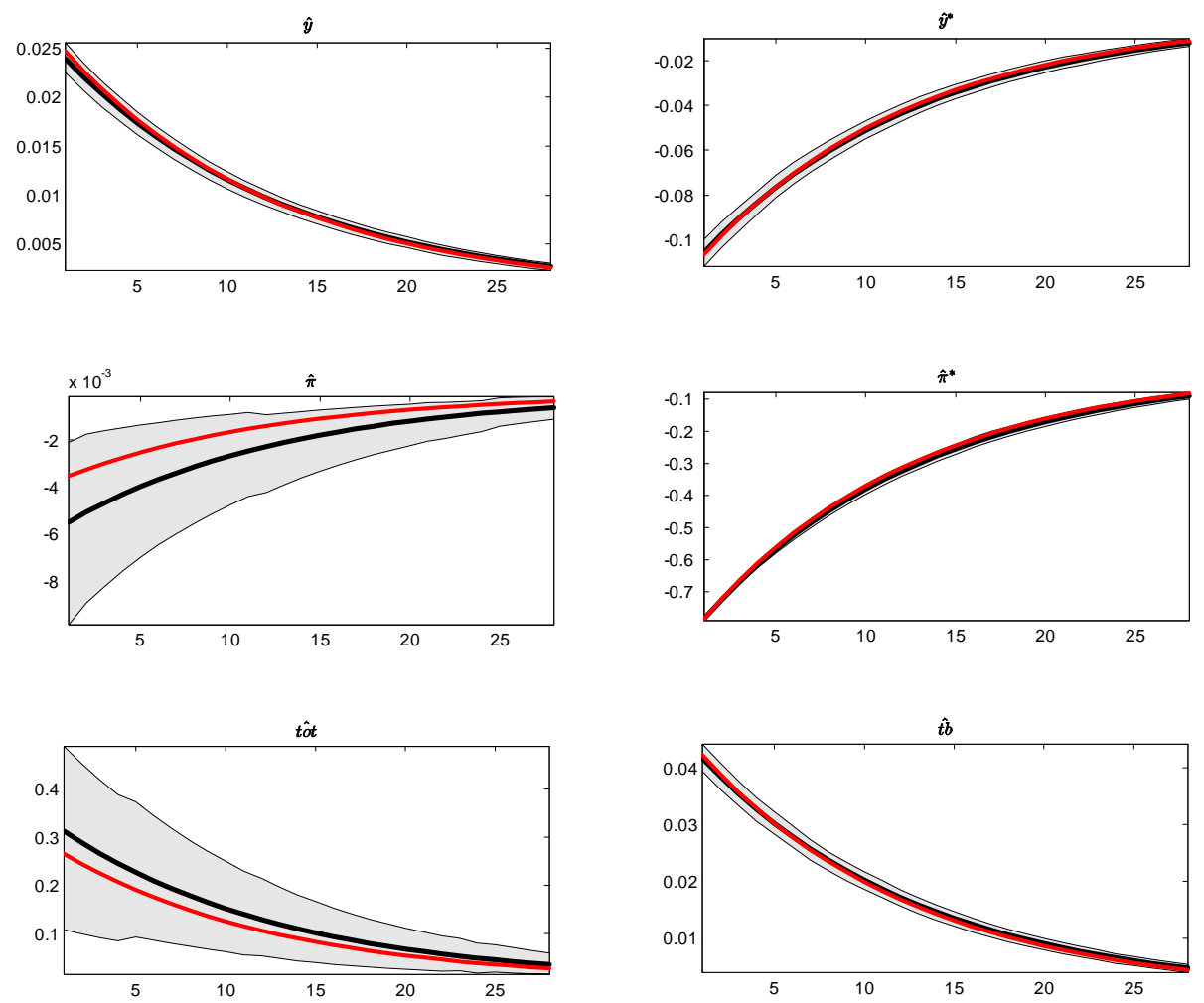

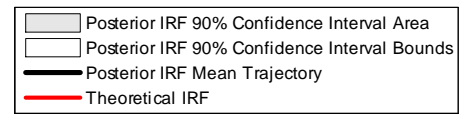

Note: The code is written for Matlab version 7.13.0.564. This figure plots the Bayesian impulse response functions (IRFs) for Home output, Foreign output, Home inflation, Foreign inflation, terms of trade and the trade balance in response to Foreign monetary shock innovations. The IRFs are estimated from the long sample of 10000 observations under the benchmark implementation of the model based on four observables (Home and Foreign output, Home and Foreign inflation). The code for this chart is available upon request from the authors. 


\section{References}

Adolfson, M., S. Laséen, J. Lindé, and M. Villani (2005). The role of sticky prices in an open economy DSGE model: A bayesian investigation. Journal of the European Economic Association 3(2-3), 444457.

Adolfson, M., S. Laséen, J. Lindé, and M. Villani (2007). Bayesian estimation of an open economy DSGE model with incomplete pass-through. Journal of International Economics 72(2), 481-511.

Adolfson, M., S. Laséen, J. Lindé, and M. Villani (2008). Evaluating an estimated new keynesian small open economy model. Journal of Economic Dynamics 32(8), 2690-2721.

Backus, D. K., P. J. Kehoe, and F. E. Kydland (1994). Dynamics of the trade balance and the terms of trade: The J-curve? American Economic Review 84(1), 84-103.

Bils, M. and P. J. Klenow (2004). Some evidence on the importance of sticky prices. Journal of Political Economy 112(5), 947-985.

Browning, M., L. P. Hansen, and J. J. Heckman (1999). Micro data and general equilibrium models. In J. B. Taylor and M. Woodford (Eds.), Handbook of Macroeconomics, vol. I, Chapter 8, pp. 543-633. Elsevier Science B.V.

Bullard, J. and K. Mitra (2007). Determinacy, learnability, and monetary policy inertia. Journal of Money, Credit and Banking 39(5), 1177-1212.

Bullard, J. and A. Singh (2008). Worldwide macroeconomic stability and monetary policy rules. Journal of Monetary Economics 55(Supplement), S34-S47.

Calvo, G. A. (1983). Staggered prices in a utility-maximizing framework. Journal of Monetary Economics 12(3), 383-398.

Canova, F. (1994). Statistical inference in calibrated models. Journal of Applied Econometrics 9 (Suppl. December), S123-S144.

Card, D. (1994). Intertemporal labor supply: An assessment. In C. Sims (Ed.), Advances in Econometrics, pp. 49-78. Cambridge, U.K.: Cambridge University Press.

Carlstrom, C. T., T. S. Fuerst, and P. Matthias (2009). Monetary policy shocks, cholesky identification, and DNK models. Journal of Monetary Economics 56(7), 1014-1021.

Chari, V. V., P. J. Kehoe, and E. R. McGrattan (2002). Can sticky price models generate volatile and persistent real exchange rates? Review of Economic Studies 69(3), 533-563.

Clarida, R., J. Galí, and M. Gertler (2002). A simple framework for international monetary policy analysis. Journal of Monetary Economics 49(5), 879-904.

Dhyne, E., L. J. Álvarez, H. Le Bihan, G. Veronese, D. Dias, J. Hoffmann, N. Jonker, P. Lünnemann, F. Rumler, and J. Vilmunen (2006). Price changes in the euro area and the united states: Some facts from individual consumer price data. Journal of Economic Perspectives 20(2), 171-192.

Engel, C. (2009). Currency misalignments and optimal monetary policy: A reexamination. NBER Working Paper no. 14829 (Cambridge, Mass., National Bureau of Economic Research, April). 
Engel, C. and J. Wang (2011). International trade in durable goods: Understanding volatility, cyclicality, and elasticities. Journal of International Economics 83(1), 37-52.

Engler, P. (2009). Gains from migration in a new keynesian framework. Unpublished paper, Freie Universität Berlin.

Evans, M. D. (1996). Peso problems: Their theoretical and empirical implications. In G. S. Maddala and C. R. Rao (Eds.), Handbook of Statistics: Statistical Methods in Finance, vol. 14, Chapter 21, pp. 613-646. Elsevier Science.

Ferroni, F. (2011). Trend agnostic one-step estimation of DSGE models. B.E. Journal of Macroeconomics, Advances 11: Iss. 1(25).

Guerron-Quintana, P. A. (2010). What you match does matter: The effects of data on DSGE estimation. Journal of Applied Econometrics 25(5), 774-804.

Hall, R. E. (1988). Intertemporal substitution in consumption. Journal of Political Economy 96(2), 339357.

Heathcote, J. and F. Perri (2002). Financial autarky and international business cycles. Journal of Monetary Economics $49(3), 601-627$.

Keane, M. P. (2010). Labor supply and taxes: A survey. School of Finance and Economics, University of Technology Sydney, Working Paper no. 160.

Klenow, P. J. and O. Kryvtsov (2008). State-dependent or time-dependent pricing: Does it matter for recent U.S. inflation? Quarterly Journal of Economics 123(3), 863-904.

Klenow, P. J. and B. A. Malin (2010). Microeconomic evidence on price-setting. In B. M. Friedman and M. Woodford (Eds.), Handbook of Monetary Economics, Volume 3, Chapter 6, pp. 231-284. Elsevier.

Leith, C. and J. Malley (2007). Estimated open economy new keynesian phillips curves for the G7. Open Economies Review 18(4), 405-426.

Lucas, R. E. (1990). Supply-side economics: An analytical review. Oxford Economic Papers 42(2), 293316.

Martínez-García, E. (2008). Globalization and monetary policy: An introduction. Federal Reserve Bank of Dallas, Globalization and Monetary Policy Institute Working Paper no. 11(April).

Martínez-García, E. and J. Søndergaard (2008). The real exchange rate in sticky price models: Does investment matter? Federal Reserve Bank of Dallas, Globalization and Monetary Policy Institute Working Paper no. 17(July).

Martínez-García, E. and M. A. Wynne (2010). The global slack hypothesis. Federal Reserve Bank of Dallas Staff Papers, 10 (September).

Nakamura, E. and J. Steinsson (2008). Five facts about prices: A reevaluation of menu cost models. Quarterly Journal of Economics 123(4), 1415-1464.

Neiss, K. S. and E. Nelson (2003). The real-interest-rate gap as an inflation indicator. Macroeconomic Dynamics 7(2), 239-262.

Neiss, K. S. and E. Nelson (2005). Inflation dynamics, marginal cost, and the output gap: Evidence from three countries. Journal of Money, Credit, and Banking 37(6), 1019-1045. 
Pencavel, J. (1986). Labor supply of men: A survey. In O. Ashenfelter and R. Layard (Eds.), Handbook of Labor Economics. Volume I, Chapter 1, pp. 3-102. Elsevier Science Publishers BV.

Rotemberg, J. J. and M. Woodford (1998a). Interest rate rules in an estimated sticky price model. In J. B. Taylor (Ed.), Monetary Policy Rules. Chicago, Illinois, USA: University of Chicago Press.

Rotemberg, J. J. and M. Woodford (1998b). An optimization-based econometric model for the evaluation of monetary policy. In B. S. Bernanke and J. J. Rotemberg (Eds.), NBER Macroeconomics Annual 1997, vol. 12, Volume 12, pp. 297-346. Cambridge, Massachusetts, USA: MIT University Press.

Rudebusch, G. D. (2002). Term structure evidence on interest rate smoothing and moneary policy inertia. Journal of Monetary Economics 49(6), 1161-1187.

Rudebusch, G. D. (2006). Monetary policy inertia: Fact or fiction? International Journal of Central Banking 2(4), 85-135.

Rumler, F. (2007). Estimates of the open economy new keynesian phillips curve for euro area countries. Open Economies Review 18(4), 427-451. September.

Ríos-Rull, J.-V., F. Schorfheide, C. Fuentes-Albero, M. Kryshko, and R. Santaeulàlia-Llopis (2011). Methods versus substance: Measuring the effects of technology shocks on hours. Mimeo, University of Pennsylvania.

Taylor, J. B. (1993). Discretion versus policy rules in practice. Carnegie-Rochester Conference Series on Public Policy 39, 195-214.

Taylor, J. B. (1999). Staggered price and wage setting in macroeconomics. In J. B. Taylor and M. Woodford (Eds.), Handbook of Macroeconomics, Vol. 1B, Volume 1B, Chapter 15, pp. 1009-1050. Amsterdam: Elsevier/North Holland.

Villemot, S. (2011). Solving rational expectations models at first order: What dynare does. Dynare Working Paper Series no. 2, CEPREMAP, April.

Woodford, M. (2003). Interest and Prices. Foundations of a Theory of Monetary Policy. Princeton, New Jersey: Princeton University Press.

Woodford, M. (2010). Globalization and monetary control. In J. Galí and M. J. Gertler. (Eds.), International Dimensions of Monetary Policy, NBER Conference Report, Chapter 1, pp. 13-77. Chicago: University of Chicago Press.

Yogo, M. (2004). Estimating the elasticity of intertemporal substitution when instruments are weak. Review of Economics and Statistics 86(3), 797-810.

Zellner, A. (1971). An Introduction to Bayesian Inference in Econometrics. John Wiley \& Sons. 